\title{
Palladium-Catalyzed Intramolecular Coupling between Aryl lodides and Allyl Moieties via Thermal and Microwave-Assisted Conditions
}

\author{
Mark Lautens*, Eiji Tayama and Christelle Herse \\ Davenport Research Laboratories, Department of Chemistry, University of Toronto, \\ Toronto, Ontario, Canada M5S $3 H 6$.
}

\section{Supporting Information}

General. Infrared (IR) spectra were recorded on a Perkin Elmer FT-IR spectrometer, SPECTRUM 1000. ${ }^{1} \mathrm{H}$ and ${ }^{13} \mathrm{C}$ NMR spectra were measured on Mercury 300, Mercury 400 and Unity 500 spectrometers. Splitting patterns are indicated as s, singlet; d, doublet; t, triplet; q, quartet; m, multiplet; br, broad peak. For thin layer chromatography (TLC) analysis throughout this work, Merck precoated TLC plates (silica gel $60 \mathrm{GF}_{254}$ on aluminium sheet) were used. The products were purified by preparative column chromatography on silica gel (60Å, ultrapure silica gel, SILICYCLE). The high-resolution mass spectra (HRMS) analysis was accomplished at the Department of Chemistry, University of Toronto (EI and ESI). Reactions were conducted in round-bottomed flasks with magnetic stirbars under an atmosphere of dry nitrogen for conventional thermal conditions. Microwave-assisted reactions were performed in Emrys Liberator model from Personal Chemistry. Reactants were filled into appropriate microwave process vials. $\operatorname{Pd}_{2}(\mathrm{dba})_{3} \cdot \mathrm{CHCl}_{3}$ was prepared according to the literature. ${ }^{1} \mathrm{Pd}(\mathrm{OAc})_{2}, \mathrm{Pd}\left(\mathrm{PPh}_{3}\right)_{4}, \mathrm{PdCl}_{2}$ (tri-o-tolylphosphine $)_{2}$ and $\mathrm{Sc}(\mathrm{OTf})_{3}$, were purchased from Strem Chemicals, Inc. 2-Methyl-2-vinyloxirane 1 was purchased from Sigma-Aldrich Canada Ltd.

2-iodoaniline and 4-Chloro-2-iodoaniline: purchased from Aldrich. 2-Iodo-4,6-dimethylaniline: prepared according to a procedure described in the literature. ${ }^{2}$ 
2-Iodo-6-methoxyaniline: $N$-(tert-Butoxycarbonyl)-2-iodo-6-methoxyaniline ${ }^{3}(1.03 \mathrm{~g}, 2.95$ mmol) was dissolved in $\mathrm{CH}_{2} \mathrm{Cl}_{2}(5.9 \mathrm{~mL})$ and treated with trifluoroacetic acid $(1.4 \mathrm{~mL}, 18$ mmol) at $0{ }^{\circ} \mathrm{C}$ and the solution was stirred for $4.5 \mathrm{~h}$ at room temperature. The volatiles were removed by evaporation and the residue was treated with saturated $\mathrm{NaHCO}_{3}$. The mixture was extracted with $\mathrm{Et}_{2} \mathrm{O}$ and the combined extracts were washed with brine, dried over $\mathrm{Na}_{2} \mathrm{SO}_{4}$ and concentrated. The residue was used without purification in next step (preparation of aldimine).

\section{Preparation of aromatic aldimine.}

General procedure: To a solution of aldehyde (1.0 equiv), aniline (1.0 equiv) and $\mathrm{Et}_{3} \mathrm{~N}$ (3.0 equiv) in $\mathrm{CH}_{2} \mathrm{Cl}_{2}(0.3 \mathrm{M})$ was added a $1.0 \mathrm{M} \mathrm{TiCl}_{4}$ solution in $\mathrm{CH}_{2} \mathrm{Cl}_{2}\left(0.6\right.$ equiv) at $0{ }^{\circ} \mathrm{C}$. After stirring for $30 \mathrm{~min}$ at the same temperature, the mixture was allowed to warm to room temperature and stirred for a few hours. Removal of $\mathrm{TiO}_{2}$ by Celite filtration and the filtrate was concentrated. To the residue was added toluene again and $\mathrm{Et}_{3} \mathrm{~N} \cdot \mathrm{HCl}$ was removed by Celite filtration. The volatiles were removed by evaporation and the residue was purified by chromatography on silica gel (hexane/Et ${ }_{2} \mathrm{O}$ as eluent) and/or recrystalization from hexane or hexane-benzene and/or Kugelrohr distillation in vacuo to afford the corresponding aldimine. The yields are noted in Table 1 .

Table 1. Preparation of Aldimines.

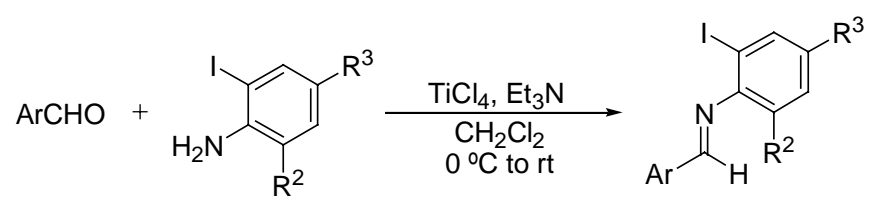

\begin{tabular}{cccccc}
\hline entry & $\mathrm{Ar}$ & $\mathrm{R}^{2}$ & $\mathrm{R}^{3}$ & \multicolumn{1}{c}{ Yield (\%) $)^{a, b}$} \\
\hline 1 & $\mathrm{Ph}$ & $\mathrm{H}$ & $\mathrm{H}$ & $\mathbf{a}$ & 87 \\
2 & $4-\mathrm{Me}-\mathrm{Ph}$ & $\mathrm{H}$ & $\mathrm{H}$ & $\mathbf{b}$ & 49 \\
3 & $4-\mathrm{Cl}-\mathrm{Ph}$ & $\mathrm{H}$ & $\mathrm{H}$ & $\mathbf{c}$ & 78 \\
4 & $4-\mathrm{Br}-\mathrm{Ph}$ & $\mathrm{H}$ & $\mathrm{H}$ & $\mathbf{d}$ & 68 \\
5 & $\mathrm{Ph}$ & $\mathrm{Me}$ & $\mathrm{Me}$ & $\mathbf{e}$ & 66 \\
6 & $\mathrm{Ph}$ & $\mathrm{H}$ & $\mathrm{Cl}$ & $\mathbf{f}$ & 69 \\
7 & $\mathrm{Ph}$ & $\mathrm{OMe}$ & $\mathrm{H}$ & $\mathbf{g}$ & 77 \\
\hline
\end{tabular}

${ }^{a}$ Isolated yield.

${ }^{b}$ The yields were determined after recrystalization. 


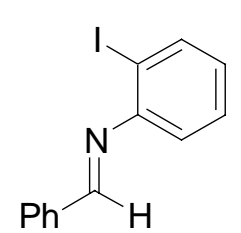

Benzylidene(2-iodophenyl)amine: ${ }^{4}$ pale yellow crystal, $\mathrm{mp}=56-57{ }^{\circ} \mathrm{C}$, purified by recrystalization from hexane; ${ }^{1} \mathrm{H}$ NMR $\left(300 \mathrm{MHz}, \mathrm{CDCl}_{3}\right) \delta 8.31$ $(1 \mathrm{H}, \mathrm{s}, \mathrm{CH}=\mathrm{N}), 7.99-7.96(2 \mathrm{H}, \mathrm{m}, \mathrm{Ar}-\mathrm{H}), 7.90(1 \mathrm{H}, \mathrm{dd}, J=7.8,1.5 \mathrm{~Hz}, \mathrm{Ar}-\mathrm{H})$, 7.53-7.46 (3H, m, Ar-H), 7.37 (1H, td, $J=7.8,1.5 \mathrm{~Hz}, \mathrm{Ar}-\mathrm{H}), 7.01(1 \mathrm{H}, \mathrm{dd}, J$ $=7.8,1.5 \mathrm{~Hz}, \mathrm{Ar}-\mathrm{H}), 6.93(1 \mathrm{H}, \mathrm{td}, J=7.8,1.5 \mathrm{~Hz}, \mathrm{Ar}-\mathrm{H}) ;{ }^{13} \mathrm{C} \mathrm{NMR}\left(75 \mathrm{MHz}, \mathrm{CDCl}_{3}\right) \delta$ 161.0, 153.1, 139.1, 135.9, 131.8, 129.4, 129.2, 128.9, 127.1, 118.4, 94.8; IR (film) 3051, 2881, 1626, 1572, 1460, 1429, 1310, 1195, 1167, 1015, 974, 879, $760 \mathrm{~cm}^{-1}$; HRMS-EI (m/z): $\left[\mathrm{M}^{+}\right]$calcd for $\mathrm{C}_{13} \mathrm{H}_{10} \mathrm{IN} 306.9858$, Found: 306.9862 .

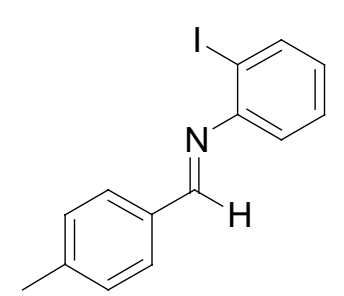

4-Methylbenzylidene(2-iodophenyl)amine: pale yellow crystal, $\mathrm{mp}=$ $33-35^{\circ} \mathrm{C}$, purified by chromatography on silica gel (hexane/ $\mathrm{Et}_{2} \mathrm{O}=15: 1$ to $10: 1$ as eluent) and then recrystalized from hexane; ${ }^{1} \mathrm{H}$ NMR (300 $\left.\mathrm{MHz}, \mathrm{CDCl}_{3}\right) \delta 8.25(1 \mathrm{H}, \mathrm{s}, \mathrm{CH}=\mathrm{N}), 7.90-7.84(3 \mathrm{H}, \mathrm{m}, \mathrm{Ar}-\mathrm{H}), 7.35(1 \mathrm{H}$, td, $J=7.8,1.5 \mathrm{~Hz}, \mathrm{Ar}-\mathrm{H}), 7.29(2 \mathrm{H}, \mathrm{d}, J=7.8 \mathrm{~Hz}, \mathrm{Ar}-\mathrm{H}), 6.99(1 \mathrm{H}, \mathrm{dd}$, $J=7.8,1.5 \mathrm{~Hz}, \mathrm{Ar}-\mathrm{H}), 6.90(1 \mathrm{H}, \mathrm{td}, J=7.8,1.5 \mathrm{~Hz}, \mathrm{Ar}-\mathrm{H}), 2.42(3 \mathrm{H}, \mathrm{s}, \mathrm{Me}) ;{ }^{13} \mathrm{C}$ NMR $(75$ $\left.\mathrm{MHz}, \mathrm{CDCl}_{3}\right) \delta 160.8,153.2,142.3,139.0,133.3,129.6,129.3,129.1,126.8,118.4,94.8$, 21.7; IR (film) 3051, 2881, 1623, 1568, 1460, 1365, 1307, 1195, 1171, 1015, 977, 882, 814, $750 \mathrm{~cm}^{-1}$; HRMS-EI (m/z): $\left[\mathrm{M}^{+}\right]$calcd for $\mathrm{C}_{14} \mathrm{H}_{12} \mathrm{IN} 321.0015$, Found: 321.0029 .

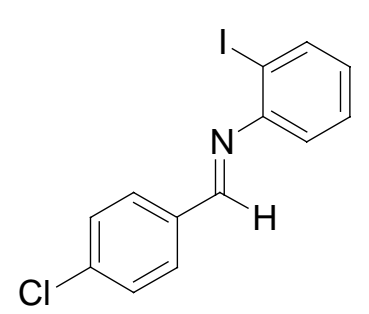

4-Chlorobenzylidene(2-iodophenyl)amine: ${ }^{4}$ pale yellow crystal, $\mathrm{mp}$ $=43-44^{\circ} \mathrm{C}$, purified by chromatography on silica gel (hexane $/ \mathrm{Et}_{2} \mathrm{O}=$ 15:1 to 10:1 as eluent) and then recrystalized from hexane; ${ }^{1} \mathrm{H}$ NMR $\left(300 \mathrm{MHz}, \mathrm{CDCl}_{3}\right) \delta 8.26(1 \mathrm{H}, \mathrm{s}, \mathrm{CH}=\mathrm{N}), 7.92-7.88(3 \mathrm{H}, \mathrm{m}, \mathrm{Ar}-\mathrm{H})$, $7.46(2 \mathrm{H}, \mathrm{dt}, J=8.4,2.1 \mathrm{~Hz}, \mathrm{Ar}-\mathrm{H}), 7.36(1 \mathrm{H}, \mathrm{td}, J=7.8,1.5 \mathrm{~Hz}$, Ar-H), $6.99(1 \mathrm{H}, \mathrm{dd}, J=7.8,1.5 \mathrm{~Hz}, \mathrm{Ar}-\mathrm{H}), 6.93(1 \mathrm{H}, \mathrm{td}, J=7.8,1.5$ $\mathrm{Hz}, \mathrm{Ar}-\mathrm{H}) ;{ }^{13} \mathrm{C}$ NMR $\left(75 \mathrm{MHz}, \mathrm{CDCl}_{3}\right) \delta 159.4,152.6,139.1,137.8,134.3,130.2,129.3$, 129.2, 127.2, 118.2, 94.9; IR (film) 3051, 2990, 2874, 1623, 1592, 1562, 1483, 1460, 1361, 1191, 1086, 1015, 882, 821, $753 \mathrm{~cm}^{-1}$; HRMS-EI (m/z): [M+] calcd for $\mathrm{C}_{13} \mathrm{H}_{9} \mathrm{CIIN} 340.9468$, Found: 340.9472.

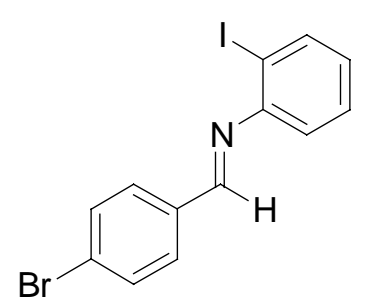

4-Bromobenzylidene(2-iodophenyl)amine: pale yellow crystal, $\mathrm{mp}$ $=50-52{ }^{\circ} \mathrm{C}$, purified by recrystalization from hexane; ${ }^{1} \mathrm{H}$ NMR $(300$ $\left.\mathrm{MHz}, \mathrm{CDCl}_{3}\right) \delta 8.26(1 \mathrm{H}, \mathrm{s}, \mathrm{CH}=\mathrm{N}), 7.90(1 \mathrm{H}, \mathrm{dd}, J=7.8,1.5 \mathrm{~Hz}$, Ar-H), $7.84(2 \mathrm{H}, \mathrm{dt}, J=8.4,2.1 \mathrm{~Hz}, \mathrm{Ar}-\mathrm{H}), 7.63(2 \mathrm{H}, \mathrm{dt}, J=8.4,2.1$ 
$\mathrm{Hz}, \mathrm{Ar}-\mathrm{H}), 7.37$ (1H, td, J=7.8, 1.5 Hz, Ar-H), 7.00 (1H, dd, J=7.8, 1.5 Hz, Ar-H), $6.94(1 \mathrm{H}$, $\mathrm{td}, J=7.8,1.5 \mathrm{~Hz}, \mathrm{Ar}-\mathrm{H}) ;{ }^{13} \mathrm{C} \mathrm{NMR}\left(75 \mathrm{MHz}, \mathrm{CDCl}_{3}\right) \delta 159.5,152.6,139.1,134.7,132.1$, 130.4, 129.3, 127.3, 126.4, 118.2, 94.8; IR (film) 3058, 2990, 2881, 1623, 1565, 1483, 1460, 1191, 1167, 1066, 1008, 882, 817, $753 \mathrm{~cm}^{-1}$; HRMS-EI (m/z): [M+] calcd for $\mathrm{C}_{13} \mathrm{H}_{9} \mathrm{BrIN}$ 384.8963, Found: 384.8968.

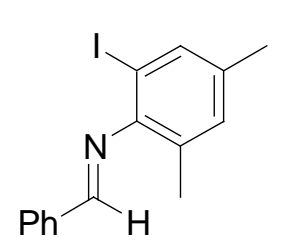

Benzylidene(2,4-dimethyl-6-iodophenyl)amine: pale yellow crystal, mp $=67-68^{\circ} \mathrm{C}$, purified by chromatography on silica gel (hexane/Et ${ }_{2} \mathrm{O}=15: 1$ as eluent) and then recrystalized from hexane; ${ }^{1} \mathrm{H}$ NMR $\left(300 \mathrm{MHz}, \mathrm{CDCl}_{3}\right)$ $\delta 8.19(1 \mathrm{H}, \mathrm{s}, \mathrm{CH}=\mathrm{N}), 7.96-7.92(2 \mathrm{H}, \mathrm{m}, \mathrm{Ar}-\mathrm{H}), 7.55-7.47(4 \mathrm{H}, \mathrm{m}, \mathrm{Ar}-\mathrm{H})$, $6.99(1 \mathrm{H}, \mathrm{s}, \mathrm{Ar}-\mathrm{H}), 2.27$ (3H, s, Me), $2.16(3 \mathrm{H}, \mathrm{s}, \mathrm{Me}) ;{ }^{13} \mathrm{C} \mathrm{NMR}\left(75 \mathrm{MHz}, \mathrm{CDCl}_{3}\right) \delta 164.8$, 150.7, 137.0, 135.6, 135.2, 131.8, 131.4, 128.8, 128.2, 89.4, 20.2, 19.1; IR (film) 3024, 2908,

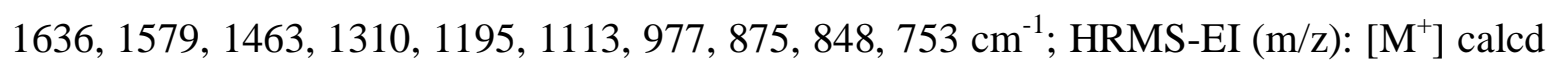
for $\mathrm{C}_{15} \mathrm{H}_{14} \mathrm{IN} 335.0171$, Found: 335.0167.

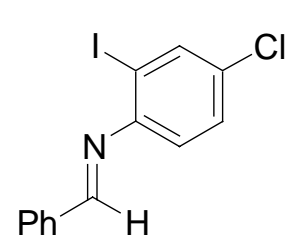

Benzylidene(4-chloro-2-iodophenyl)amine: pale yellow crystal, $\mathrm{mp}=$ $52-53{ }^{\circ} \mathrm{C}$, purified by chromatography on silica gel (hexane/Et $2 \mathrm{O}=15: 1$ to 10:1 as eluent) and then recrystalized from hexane; ${ }^{1} \mathrm{H}$ NMR $(300 \mathrm{MHz}$, $\left.\mathrm{CDCl}_{3}\right) \delta 8.28(1 \mathrm{H}, \mathrm{s}, \mathrm{CH}=\mathrm{N}), 7.97-7.94(2 \mathrm{H}, \mathrm{m}, \mathrm{Ar}-\mathrm{H}), 7.88(1 \mathrm{H}, \mathrm{d}, J=$ $2.4 \mathrm{~Hz}, \mathrm{Ar}-\mathrm{H}), 7.56-7.46(3 \mathrm{H}, \mathrm{m}, \mathrm{Ar}-\mathrm{H}), 7.34(1 \mathrm{H}, \mathrm{dd}, J=8.4,2.4 \mathrm{~Hz}, \mathrm{Ar}-\mathrm{H}), 6.93(1 \mathrm{H}, \mathrm{d}, J$ $=8.4 \mathrm{~Hz}, \mathrm{Ar}-\mathrm{H}) ;{ }^{13} \mathrm{C} \mathrm{NMR}\left(75 \mathrm{MHz}, \mathrm{CDCl}_{3}\right) \delta 161.3,151.7,138.3,135.6,132.0,131.4$, 129.3, 129.2, 128.9, 118.7, 95.1; IR (film) 3065, 3024, 2881, 1626, 1575, 1453, 1368, 1310, 1191, 1093, 1032, 970, 872, 811, $753 \mathrm{~cm}^{-1}$; HRMS-EI $(\mathrm{m} / \mathrm{z}):\left[\mathrm{M}^{+}\right]$calcd for $\mathrm{C}_{13} \mathrm{H}_{9} \mathrm{CIIN}$ 340.9468, Found: 340.9465.

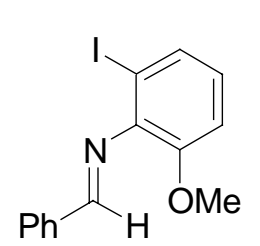

Benzylidene(2-iodo-6-methoxyphenyl)amine: yellow crystal, $\mathrm{mp}=70-71$ ${ }^{\circ} \mathrm{C}$, purified by chromatography on silica gel (hexane/Et ${ }_{2} \mathrm{O}=15: 1$ to $10: 1$ as eluent) and then recrystalized from hexane; ${ }^{1} \mathrm{H} \mathrm{NMR}\left(300 \mathrm{MHz}, \mathrm{CDCl}_{3}\right) \delta$ $8.38(1 \mathrm{H}, \mathrm{s}, \mathrm{CH}=\mathrm{N}), 8.00-7.96(2 \mathrm{H}, \mathrm{m}, \mathrm{Ar}-\mathrm{H}), 7.53-7.46(4 \mathrm{H}, \mathrm{m}, \mathrm{Ar}-\mathrm{H}), 6.94$ $(1 \mathrm{H}, \mathrm{dd}, J=8.1,0.9 \mathrm{~Hz}, \mathrm{Ar}-\mathrm{H}), 6.82(1 \mathrm{H}, \mathrm{t}, J=8.1 \mathrm{~Hz}, \mathrm{Ar}-\mathrm{H}), 3.77\left(3 \mathrm{H}, \mathrm{s}, \mathrm{OCH}_{3}\right) ;{ }^{13} \mathrm{C} \mathrm{NMR}$ $\left(75 \mathrm{MHz}, \mathrm{CDCl}_{3}\right) \delta 165.4,149.7,142.8,136.0,131.7,130.9,128.9,128.7,126.2,112.0,93.7$, 56.1; IR (film) 3058, 3003, 2935, 2826, 1630, 1575, 1558, 1456, 1426, 1310, 1252, 1191, 1028, 872, $753 \mathrm{~cm}^{-1}$; HRMS-EI $(\mathrm{m} / \mathrm{z})$ : $\left[\mathrm{M}^{+}\right]$calcd for $\mathrm{C}_{14} \mathrm{H}_{12} \mathrm{INO} 336.9964$, Found: 336.9966.

Preparation of 2,4-dimethyl-6-iodoiminoacetic acid cyclohexyl ester: A mixture of 
L-tartalic acid ( $3.0 \mathrm{~g}, 20 \mathrm{mmol})$, cyclohexanol $(6.3 \mathrm{~mL}, 60 \mathrm{mmol})$ and $p-\mathrm{TsOH} \cdot \mathrm{H}_{2} \mathrm{O}(0.19 \mathrm{~g}$, $1.0 \mathrm{mmol}$ ) in benzene ( $20 \mathrm{~mL}$ ) was refluxed for $15 \mathrm{~h}$ while removing $\mathrm{H}_{2} \mathrm{O}$ by Dean-Stark trap. The resulting mixture was cooled to room temperature and concentrated. The residue was purified by Kugelrohr distillation to give dicyclohexyl tartrate as a viscous colorless oil (6.05 g, $19.2 \mathrm{mmol}, 96 \%$ yield). The spectroscopic data was assigned with a literature. ${ }^{5}$ To a solution of dicyclohexyl tartrate $(943 \mathrm{mg}, 3.0 \mathrm{mmol})$ in THF $(15 \mathrm{~mL})$ was added $\mathrm{H}_{5} \mathrm{IO}_{6}(684$ $\mathrm{mg}, 3.0 \mathrm{mmol}$ ) at $0^{\circ} \mathrm{C}$ and the mixture was stirred for $2 \mathrm{~h}$ at room temperature. The resulting white suspension was filtered through a pad of Celite and the filtrate was concentrated to obtain a crude cyclohexyl glyoxalate. ${ }^{5}$ The crude product was used without purification in next step. To the solution of crude cyclohexyl glyoxalate $(\sim 6.0 \mathrm{mmol})$ and 2,4-dimethyl-6-iodoaniline ${ }^{2}(1.24 \mathrm{~g}, 5.0 \mathrm{mmol})$ in $\mathrm{CH}_{2} \mathrm{Cl}_{2}(10 \mathrm{~mL})$ was added $\mathrm{MgSO}_{4}(3.0 \mathrm{~g}$, $25 \mathrm{mmol}$ ) at room temperature. After stirring for $20 \mathrm{~h}$, the resulting mixture was filtered and the volatiles were removed by evaporation. Purification of the residue by chromatography on silica gel (hexane/ $\mathrm{Et}_{2} \mathrm{O}=10: 1$ to $6: 1$ as eluent) and then, recrystalization from hexane gave 2,4-dimethyl-6-iodoiminoacetic acid cyclohexyl ester as pale yellow crystals (1.22 g, $3.17 \mathrm{mmol}, 63 \%$ yield $) . \quad \mathrm{mp}=73-74{ }^{\circ} \mathrm{C} ;{ }^{1} \mathrm{H} \mathrm{NMR}\left(300 \mathrm{MHz}, \mathrm{CDCl}_{3}\right) \delta 7.66(1 \mathrm{H}, \mathrm{s}, \mathrm{CH}=\mathrm{N})$, $7.53(1 \mathrm{H}, \mathrm{s}, \mathrm{Ar}-\mathrm{H}), 6.98(1 \mathrm{H}, \mathrm{s}, \mathrm{Ar}-\mathrm{H}), 5.05(1 \mathrm{H}, \mathrm{tt}, J=9.6,4.2 \mathrm{~Hz}, \mathrm{OCH}), 2.26(3 \mathrm{H}, \mathrm{s}, \mathrm{Me})$, 2.14 (3H, s, Me), 2.04-1.97 (2H, m, $c$-Hex), 1.85-1.77 (2H, m, $c$-Hex), 1.67-1.55 (3H, m, $c$-Hex), 1.50-1.24 (3H, m, $c$-Hex); ${ }^{13} \mathrm{C}$ NMR $\left(75 \mathrm{MHz}, \mathrm{CDCl}_{3}\right) \delta$ 162.0, 157.5, 148.6, 137.3, 136.6, 131.6, 127.5, 87.5, 75.0, 31.5, 25.3, 23.8, 20.2, 19.0; IR (film) 2935, 2853, 1742, 1718, 1446, 1290, 1201, 1120, 1008, 981, 851, $780 \mathrm{~cm}^{-1}$; HRMS-EI $(\mathrm{m} / \mathrm{z}):\left[\mathrm{M}^{+}\right]$calcd for $\mathrm{C}_{16} \mathrm{H}_{20} \mathrm{INO}_{2}$ 385.0539, Found: 385.0540 .

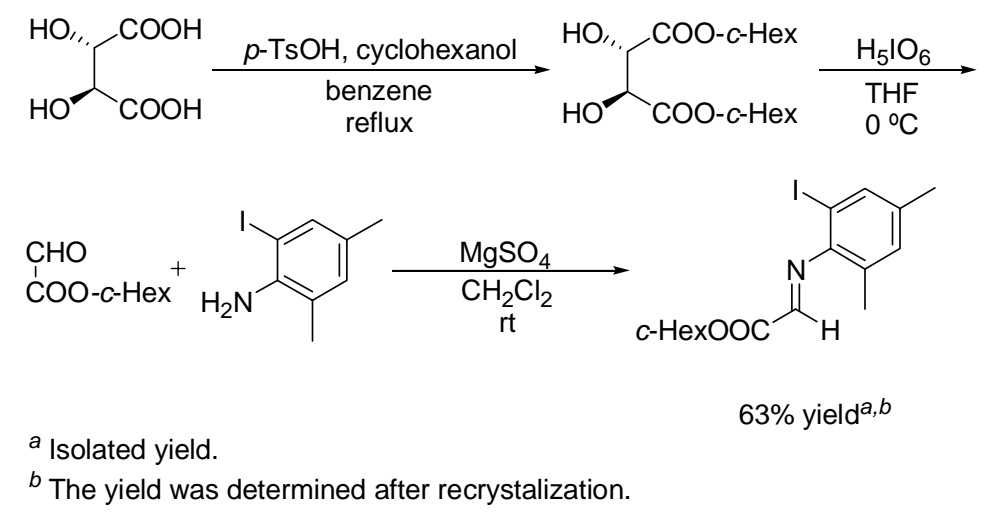

Scheme 1. Preparation of $\alpha$-Imino Cyclohexyl Ester. 
Preparation of amino aldehyde 2 by direct vinylogous Mannich-type reaction: To a solution of imine $(0.50 \mathrm{mmol})$ and vinyloxirane $(74 \mu \mathrm{L}, 0.75 \mathrm{mmol})$ in THF $(1.0 \mathrm{~mL})$ was added $\mathrm{Sc}(\mathrm{OTf})_{3}(25 \mathrm{mg}, 0.050 \mathrm{mmol}, 10 \mathrm{~mol} \%)$ at $0{ }^{\circ} \mathrm{C}$ and the mixture was stirred for the time noted in Table 2. The resulting mixture was quenched by addition of saturated $\mathrm{NaHCO}_{3}$ and extracted with $\mathrm{Et}_{2} \mathrm{O}$. The combined extracts were washed with brine, dried over $\mathrm{Na}_{2} \mathrm{SO}_{4}$ and concentrated. Purification of the residue by column chromatography on silica gel gave the corresponding amino aldehydes 2 . The reaction time, temperature and yields were noted in Table 2.

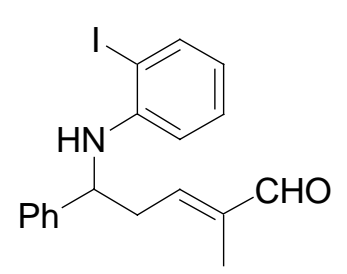

(2E)- $N$-(2-Iodophenyl)-5-amino-2-methyl-5-phenylpent-2-en-1-nal : colorless viscous oil; ${ }^{1} \mathrm{H}$ NMR $\left(300 \mathrm{MHz}, \mathrm{CDCl}_{3}\right) \delta 9.42(1 \mathrm{H}, \mathrm{s}$, CHO), 7.64 (1H, dd, $J=7.8,1.5 \mathrm{~Hz}, \mathrm{Ar}-\mathrm{H}), 7.39-7.24$ (5H, m, Ar-H), $7.03(1 \mathrm{H}, \mathrm{td}, J=7.8,1.5 \mathrm{~Hz}, \mathrm{Ar}-\mathrm{H}), 6.51(1 \mathrm{H}, \mathrm{tq}, J=7.2,1.2 \mathrm{~Hz}, 3-\mathrm{CH})$, $6.41(1 \mathrm{H}, \mathrm{td}, J=7.8,1.5 \mathrm{~Hz}, \mathrm{Ar}-\mathrm{H}), 6.33(1 \mathrm{H}, \mathrm{dd}, J=7.8,1.5 \mathrm{~Hz}, \mathrm{Ar}-\mathrm{H})$, 4.63-4.58 (2H, m, NH and 5-CH), $2.92\left(2 \mathrm{H}, \mathrm{dd}, J=7.2,6.6 \mathrm{~Hz}, 4-\mathrm{CH}_{2}\right), 1.78(3 \mathrm{H}, \mathrm{d}, J=1.2$ $\left.\mathrm{Hz}, 2-\mathrm{CH}_{3}\right) ;{ }^{13} \mathrm{C} \mathrm{NMR}\left(75 \mathrm{MHz}, \mathrm{CDCl}_{3}\right) \delta 194.7,148.5,145.7,141.8,141.7,138.9,129.3$, 128.9, 127.7, 126.1, 119.3, 112.1, 85.9, 57.7, 38.0, 9.6; IR (film) 3379, 3058, 3024, 2819,

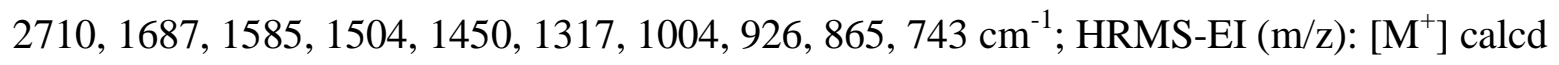
for $\mathrm{C}_{18} \mathrm{H}_{18} \mathrm{INO} 391.0433$, Found: 391.0429.

\begin{tabular}{|c|c|c|c|c|c|c|c|}
\hline \multirow[b]{2}{*}{ entry } & \multirow[b]{2}{*}{$\mathrm{R}^{1}$} & \multirow{2}{*}{$\begin{array}{r}\mathrm{R}^{3} \\
\mathrm{R}^{2}\end{array}$} & \multicolumn{2}{|c|}{$\overbrace{\begin{array}{c}\mathrm{T} \text { THF } \\
\text { temp, time }\end{array}}^{10 \mathrm{~mol} \% \mathrm{Sc}(\mathrm{OT}}$} & & $R^{3}$ & \multirow[b]{2}{*}{$\begin{array}{l}\text { Yield }^{a} \\
(\%)\end{array}$} \\
\hline & & & $\mathrm{R}^{3}$ & & $\begin{array}{c}\text { temp } \\
\left({ }^{\circ} \mathrm{C}\right)\end{array}$ & $\begin{array}{l}\text { time } \\
\text { (h) }\end{array}$ & \\
\hline 1 & $\mathrm{Ph}$ & $\mathrm{H}$ & $\mathrm{H}$ & a & 0 & 1 & 91 \\
\hline 2 & 4-Me-Ph & $\mathrm{H}$ & $\mathrm{H}$ & b & 0 & 1 & 88 \\
\hline 3 & 4-Cl-Ph & $\mathrm{H}$ & H & c & 0 & 1 & 85 \\
\hline 4 & 4-Br-Ph & $\mathrm{H}$ & $\mathrm{H}$ & d & 0 & 1 & 87 \\
\hline 5 & $\mathrm{Ph}$ & $\mathrm{Me}$ & $\mathrm{Me}$ & e & 0 & 1 & 91 \\
\hline 6 & $\mathrm{Ph}$ & $\mathrm{H}$ & $\mathrm{Cl}$ & f & 0 & 1 & 87 \\
\hline 7 & $\mathrm{Ph}$ & $\mathrm{OMe}$ & $\mathrm{H}$ & g & 0 & 1 & 88 \\
\hline 8 & COO-c-Hex & $\mathrm{Me}$ & $\mathrm{Me}$ & h & 0 addition to 50 & 0.5 & 80 \\
\hline
\end{tabular}




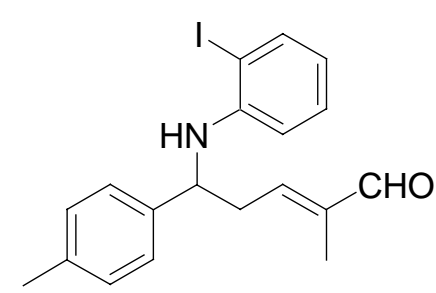

(2E)-N-(2-Iodophenyl)-5-amino-2-methyl-5-(4-methylphenyl )pent-2-en-1-nal: colorless viscous oil; ${ }^{1} \mathrm{H}$ NMR $(300 \mathrm{MHz}$, $\left.\mathrm{CDCl}_{3}\right) \delta 9.41(1 \mathrm{H}, \mathrm{s}, \mathrm{CHO}), 7.64(1 \mathrm{H}, \mathrm{dd}, J=7.8,1.5 \mathrm{~Hz}, \mathrm{Ar}-\mathrm{H})$, $7.23(2 \mathrm{H}, \mathrm{d}, J=8.1 \mathrm{~Hz}, \mathrm{Ar}-\mathrm{H}), 7.15(2 \mathrm{H}, \mathrm{d}, J=8.1 \mathrm{~Hz}, \mathrm{Ar}-\mathrm{H})$, $7.03(1 \mathrm{H}, \mathrm{td}, J=7.8,1.5 \mathrm{~Hz}, \mathrm{Ar}-\mathrm{H}), 6.51(1 \mathrm{H}, \mathrm{tq}, J=7.5,1.2 \mathrm{~Hz}$, 3-CH), $6.41(1 \mathrm{H}, \mathrm{td}, J=7.8,1.5 \mathrm{~Hz}, \mathrm{Ar}-\mathrm{H}), 6.34(1 \mathrm{H}, \mathrm{dd}, J=7.8,1.5 \mathrm{~Hz}, \mathrm{Ar}-\mathrm{H}), 4.61-4.55$ $\left(2 \mathrm{H}, \mathrm{m}, \mathrm{NH}\right.$ and 5-CH), $2.90\left(2 \mathrm{H}, \mathrm{dd}, J=7.5,6.6 \mathrm{~Hz}, 4-\mathrm{CH}_{2}\right), 2.33\left(3 \mathrm{H}, \mathrm{s}, \mathrm{Ar}-\mathrm{CH}_{3}\right), 1.78(3 \mathrm{H}$, d, $\left.J=1.2 \mathrm{~Hz}, 2-\mathrm{CH}_{3}\right) ;{ }^{13} \mathrm{C} \mathrm{NMR}\left(75 \mathrm{MHz}, \mathrm{CDCl}_{3}\right) \delta 194.8,148.8,148.7,145.8,141.7,139$ (m), 138.6, 137.3, 129.6, 129.3, 126.0, 119 (m), 112 (m), 85.9, 57.3, 38.0, 21.1, 21.0, 9.6; IR (film) 3379, 3010, 2915, 2710, 1684, 1582, 1504, 1450, 1314, 1079, 1004, 923, 814, 743 $\mathrm{cm}^{-1}$; HRMS-EI (m/z): [ $\left.\mathrm{M}^{+}\right]$calcd for $\mathrm{C}_{19} \mathrm{H}_{20} \mathrm{INO}$ 405.0590, Found: 405.0586.

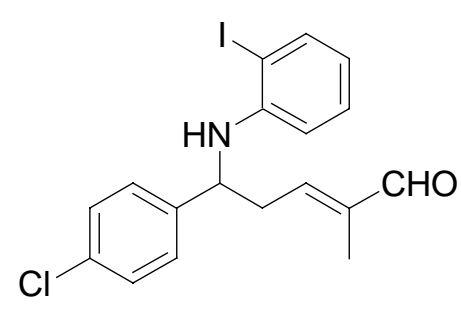

(2E)-N-(2-Iodophenyl)-5-amino-2-methyl-5-(4-chloropheny l)pent-2-en-1-nal: colorless viscous oil; ${ }^{1} \mathrm{H}$ NMR $(300 \mathrm{MHz}$, $\left.\mathrm{CDCl}_{3}\right) \delta 9.42(1 \mathrm{H}, \mathrm{s}, \mathrm{CHO}), 7.65(1 \mathrm{H}, \mathrm{dd}, J=7.8,1.5 \mathrm{~Hz}$, Ar-H), $7.33(2 \mathrm{H}, \mathrm{dt}, J=6.5,2.1 \mathrm{~Hz}, \mathrm{Ar}-\mathrm{H}), 7.28(2 \mathrm{H}, \mathrm{dt}, J=6.5$, $2.1 \mathrm{~Hz}, \mathrm{Ar}-\mathrm{H}), 7.04(1 \mathrm{H}, \mathrm{td}, J=7.8,1.5 \mathrm{~Hz}, \mathrm{Ar}-\mathrm{H}), 6.49(1 \mathrm{H}, \mathrm{tq}$, $J=7.5,0.9 \mathrm{~Hz}, 3-\mathrm{CH}), 6.43(1 \mathrm{H}, \mathrm{td}, J=7.8,1.5 \mathrm{~Hz}, \mathrm{Ar}-\mathrm{H}), 6.27$ $(1 \mathrm{H}, \mathrm{dd}, J=7.8,1.5 \mathrm{~Hz}, \mathrm{Ar}-\mathrm{H}), 4.61-4.55(2 \mathrm{H}, \mathrm{m}, \mathrm{NH}$ and 5-CH), $2.90(2 \mathrm{H}, \mathrm{dd}, J=7.5,6.9$ $\left.\mathrm{Hz}, 4-\mathrm{CH}_{2}\right), 1.80\left(3 \mathrm{H}, \mathrm{d}, J=0.9 \mathrm{~Hz}, 2-\mathrm{CH}_{3}\right) ;{ }^{13} \mathrm{C} \mathrm{NMR}\left(75 \mathrm{MHz}, \mathrm{CDCl}_{3}\right) \delta 194.6,147.8$, 145.4, 142.1, 140.3, 139.0, 133.4, 129.3, 129.1, 127.5, 119.6, 112.1, 85.9, 57.2, 37.8, 9.7; IR (film) 3379, 3058, 2826, 2710, 1684, 1585, 1500, 1450, 1314, 1086, 1004, 923, 824, 743 $\mathrm{cm}^{-1}$; HRMS-ESI (m/z): $[\mathrm{M}+\mathrm{H}]^{+}$calcd for $\mathrm{C}_{18} \mathrm{H}_{18} \mathrm{CIINO} 426.0116$, Found: 426.0130 .

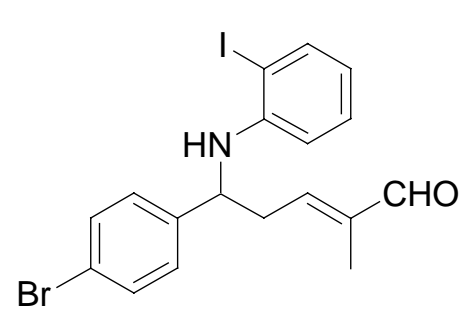

(2E)-N-(2-Iodophenyl)-5-amino-2-methyl-5-(4-bromopheny 1)pent-2-en-1-nal: yellow vicous oil; ${ }^{1} \mathrm{H}$ NMR $(300 \mathrm{MHz}$, $\left.\mathrm{CDCl}_{3}\right) \delta 9.42(1 \mathrm{H}, \mathrm{s}, \mathrm{CHO}), 7.65(1 \mathrm{H}, \mathrm{dd}, J=7.8,1.2 \mathrm{~Hz}$, Ar-H), $7.48(2 \mathrm{H}, \mathrm{dt}, J=8.7,2.4 \mathrm{~Hz}, \mathrm{Ar}-\mathrm{H}), 7.23(2 \mathrm{H}, \mathrm{dt}, J=8.7$, $2.4 \mathrm{~Hz}, \mathrm{Ar}-\mathrm{H}), 7.03(1 \mathrm{H}, \mathrm{td}, J=7.8,1.2 \mathrm{~Hz}, \mathrm{Ar}-\mathrm{H}), 6.48(1 \mathrm{H}, \mathrm{tq}$, $J=7.5,1.2 \mathrm{~Hz}, 3-\mathrm{CH}), 6.43(1 \mathrm{H}, \mathrm{td}, J=7.8,1.2 \mathrm{~Hz}, \mathrm{Ar}-\mathrm{H}), 6.26$ $(1 \mathrm{H}, \mathrm{dd}, J=7.8,1.2 \mathrm{~Hz}, \mathrm{Ar}-\mathrm{H}), 4.61-4.53\left(2 \mathrm{H}, \mathrm{m}, \mathrm{NH}\right.$ and 5-CH), 2.88-2.81 (2H, m, 4-CH $\mathrm{CH}_{2}$, $1.80\left(3 \mathrm{H}, \mathrm{d}, J=1.2 \mathrm{~Hz}, 2-\mathrm{CH}_{3}\right) ;{ }^{13} \mathrm{C} \mathrm{NMR}\left(75 \mathrm{MHz}, \mathrm{CDCl}_{3}\right) \delta 194.5,147.7,145.4,142.1$, 140.8, 139.0, 132.1, 129.3, 127.9, 121.4, 119.6, 112.0, 85.9, 57.2, 37.8, 9.7; IR (film) 3386, $3058,2819,2710,1681,1585,1500,1453,1314,1072,1008,926,821,743 \mathrm{~cm}^{-1}$; HRMS-EI $(\mathrm{m} / \mathrm{z}):\left[\mathrm{M}^{+}\right]$calcd for $\mathrm{C}_{18} \mathrm{H}_{17} \mathrm{BrINO} 468.9538$, Found: 468.9537 . 


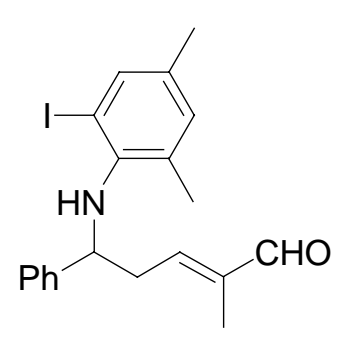

(2E)-N-(2,4-Dimethyl-6-iodophenyl)-5-amino-2-methyl-5-phenylpe nt-2-en-1-nal: colorless viscous oil; ${ }^{1} \mathrm{H}$ NMR $\left(300 \mathrm{MHz}, \mathrm{CDCl}_{3}\right) \delta$ $9.28(1 \mathrm{H}, \mathrm{s}, \mathrm{CHO}), 7.41(1 \mathrm{H}, \mathrm{d}, J=0.9 \mathrm{~Hz}, \mathrm{Ar}-\mathrm{H}), 7.35-7.23(5 \mathrm{H}, \mathrm{m}$, Ar-H), $6.85(1 \mathrm{H}, \mathrm{d}, J=0.9 \mathrm{~Hz}, \operatorname{Ar}-\mathrm{H}), 6.42(1 \mathrm{H}, \mathrm{tq}, J=7.2,1.2 \mathrm{~Hz}$, $3-\mathrm{CH}), 4.51(1 \mathrm{H}, \mathrm{t}, J=6.9 \mathrm{~Hz}, 5-\mathrm{CH}), 3.78(1 \mathrm{H}, \mathrm{s}, \mathrm{NH}), 3.03-2.86(2 \mathrm{H}$, m, 4- $\left.\mathrm{CH}_{2}\right), 2.20\left(3 \mathrm{H}, \mathrm{s}, \mathrm{Ar}-\mathrm{CH}_{3}\right), 2.18\left(3 \mathrm{H}, \mathrm{s}, \mathrm{Ar}-\mathrm{CH}_{3}\right), 1.68(3 \mathrm{H}, \mathrm{d}, J=$ $\left.1.2 \mathrm{~Hz}, 2-\mathrm{CH}_{3}\right) ;{ }^{13} \mathrm{C} \mathrm{NMR}\left(75 \mathrm{MHz}, \mathrm{CDCl}_{3}\right) \delta 195.0,150.3,143.3,141.7,140.5,137.5$, 133.6, 132.8, 130.3, 128.6, 127.7, 126.9, 95.9, 61.1, 36.2, 20.1, 19.9, 9.4; IR (film) 3338, 3024, 2915, 2710, 1684, 1640, 1473, 1436, 1266, 1232, 1055, 1025, 851, $760 \mathrm{~cm}^{-1}$; HRMS-EI (m/z): $\left[\mathrm{M}^{+}\right]$calcd for $\mathrm{C}_{20} \mathrm{H}_{22} \mathrm{INO} 419.0746$, Found: 419.0755 .

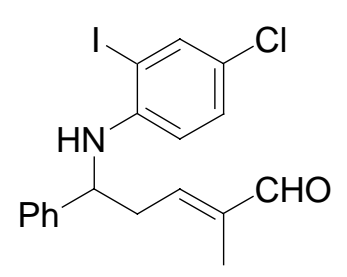

(2E)- $N$-(4-Chloro-4-iodophenyl)-5-amino-2-methyl-5-chloropheny lpent-2-en-1-nal: colorless viscous oil; ${ }^{1} \mathrm{H} \mathrm{NMR}\left(300 \mathrm{MHz}, \mathrm{CDCl}_{3}\right) \delta$ $9.42(1 \mathrm{H}, \mathrm{s}, \mathrm{CHO}), 7.61(1 \mathrm{H}, \mathrm{d}, J=2.1 \mathrm{~Hz}, \mathrm{Ar}-\mathrm{H}), 7.39-7.26(5 \mathrm{H}, \mathrm{m}$, $\mathrm{Ar}-\mathrm{H}), 6.99(1 \mathrm{H}, \mathrm{dd}, J=8.7,2.4 \mathrm{~Hz}, \mathrm{Ar}-\mathrm{H}), 6.49(1 \mathrm{H}, \mathrm{tq}, J=7.5,1.2 \mathrm{~Hz}$,

$3-\mathrm{CH}), 6.21(1 \mathrm{H}, \mathrm{d}, J=8.7 \mathrm{~Hz}, \mathrm{Ar}-\mathrm{H}), 4.62-4.54(2 \mathrm{H}, \mathrm{m}, \mathrm{NH}$ and 5-CH), 3.00-2.84 (2H, m, 4- $\left.\mathrm{CH}_{2}\right), 1.78\left(3 \mathrm{H}, \mathrm{d}, J=1.2 \mathrm{~Hz}, 2-\mathrm{CH}_{3}\right) ;{ }^{13} \mathrm{C} \mathrm{NMR}(75 \mathrm{MHz}$, $\left.\mathrm{CDCl}_{3}\right) \delta 194.65,194.60,148.1,148.0,144.5,142.0,141.2,137.9,137.7,129(\mathrm{~m}), 128(\mathrm{~m})$, 126 (m), 122.7, 112.4, 112.2, 85.2, 57.8, 37.9, 9.7; IR (film) 3386, 3058, 3024, 2819, 2710, 1687, 1582, 1494, 1453, 1385, 1310, 1103, 1021, 868, 800, $756 \mathrm{~cm}^{-1}$; HRMS-ESI (m/z): $[\mathrm{M}+\mathrm{H}]^{+}$calcd for $\mathrm{C}_{18} \mathrm{H}_{18} \mathrm{ClINO} 426.0116$, Found: 426.0128 .

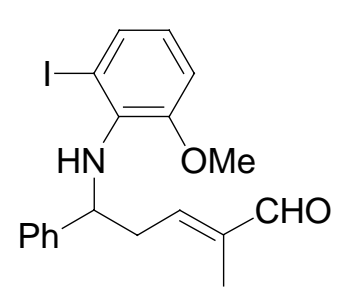

(2E)- $N$-(2-Iodo-6-methoxyphenyl)-5-amino-2-methyl-5-phenylpent -2-en-1-nal: pale yellow viscous oil; ${ }^{1} \mathrm{H} \mathrm{NMR}\left(300 \mathrm{MHz}, \mathrm{CDCl}_{3}\right) \delta$ $9.33(1 \mathrm{H}, \mathrm{s}, \mathrm{CHO}), 7.32-7.18$ (6H, m, Ar-H), $6.71(1 \mathrm{H}, \mathrm{dd}, J=8.1,1.2$ $\mathrm{Hz}, \mathrm{Ar}-\mathrm{H}), 6.58-6.52$ (2H, m, Ar-H and 3-CH), 5.07 (1H, br, 5-CH), $4.21(1 \mathrm{H}, \mathrm{s}, \mathrm{NH}), 3.71\left(3 \mathrm{H}, \mathrm{s}, \mathrm{OCH}_{3}\right), 2.94(1 \mathrm{H}, \mathrm{dddd}, J=15.0,15.0$, 7.2, $\left.0.9 \mathrm{~Hz}, 4-\mathrm{CH}_{2}\right), 2.87\left(1 \mathrm{H}, \mathrm{dddd}, J=15.0,15.0,7.2,0.9 \mathrm{~Hz}, 4-\mathrm{CH}_{2}\right), 1.71(3 \mathrm{H}, \mathrm{d}, J=1.2$ $\left.\mathrm{Hz}, 2-\mathrm{CH}_{3}\right) ;{ }^{13} \mathrm{C} \mathrm{NMR}\left(75 \mathrm{MHz}, \mathrm{CDCl}_{3}\right) \delta 195.1,150.7,150.6,142.3,140.6,137.2,131.5$, 128.4, 127.4, 126.7, 123.0, 111.7, 92.1, 59.9, 55.7, 36.9, 9.4; IR (film) 3338, 3024, 2935, 2826, 2710, 1684, 1575, 1463, 1229, 1086, 1025, 865, $760 \mathrm{~cm}^{-1}$; HRMS-ESI (m/z): [M+H] ${ }^{+}$ calcd for $\mathrm{C}_{19} \mathrm{H}_{21} \mathrm{INO}_{2} 422.0611$, Found: 422.0614 . 


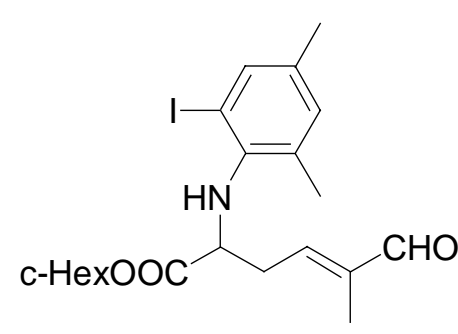

(2E)-N-(2,4-Dimethyl-6-iodophenyl)-5-amino-5-cyclohexylc arbonyl-2-methylpent-2-en-1-nal: colorless viscous oil; ${ }^{1} \mathrm{H}$ NMR $\left(300 \mathrm{MHz}, \mathrm{CDCl}_{3}\right) \delta 9.39(1 \mathrm{H}, \mathrm{s}, \mathrm{CHO}), 7.44(1 \mathrm{H}, \mathrm{s}$, Ar-H), 6.89 (1H, s, Ar-H), 6.50 (1H, tq, $J=7.2,1.2 \mathrm{~Hz}, 3-\mathrm{CH})$, 4.84-4.75 (1H, m, OCH), 4.29 (1H, dt, $J=10.5,6.3 \mathrm{~Hz}, 5-\mathrm{CH})$, $4.18(1 \mathrm{H}, \mathrm{d}, J=10.5 \mathrm{~Hz}, \mathrm{NH}), 2.88-2.72\left(2 \mathrm{H}, \mathrm{m}, 4-\mathrm{CH}_{2}\right), 2.30$ $\left(3 \mathrm{H}, \mathrm{s}, \mathrm{Ar}-\mathrm{CH}_{3}\right), 2.20\left(3 \mathrm{H}, \mathrm{s}, \mathrm{Ar}-\mathrm{CH}_{3}\right), 1.82-1.20(10 \mathrm{H}, \mathrm{m}, c-\mathrm{Hex}), 1.69$ (3H, d, J = $1.2 \mathrm{~Hz}$, $\left.2-\mathrm{CH}_{3}\right) ;{ }^{13} \mathrm{C} \mathrm{NMR}\left(75 \mathrm{MHz}, \mathrm{CDCl}_{3}\right) \delta 194.7,172.1,148.0,142.6,141.3,137.8,133.7,132.7$, 129.8, 94.4, 74.1, 58.7, 32.7, 31.5, 25.2, 23.6, 19.9, 19.8, 9.4; IR (film) 3331, 2928, 2853, 2710, 1732, 1687, 1473, 1446, 1337, 1273, 1195, 1123, 1011, 855, $783 \mathrm{~cm}^{-1}$; HRMS-ESI $(\mathrm{m} / \mathrm{z}):[\mathrm{M}+\mathrm{H}]^{+}$calcd for $\mathrm{C}_{21} \mathrm{H}_{29} \mathrm{INO}_{3} 470.1186$, Found: 470.1190 .

Preparation of allyl alcohol or allylic ester 1 from amino aldehyde : A amino aldehyde (1.0 equiv) was dissolved in $\mathrm{MeOH}(0.2 \mathrm{M})$ and treated with $\mathrm{NaBH}_{4}\left(1.0\right.$ equiv) at $0{ }^{\circ} \mathrm{C}$. The mixture was stirred for $30 \mathrm{~min}$ at the same temperature and for over $1 \mathrm{~h}$ at room temperature. The reactant was diluted with water and extracted with $\mathrm{Et}_{2} \mathrm{O}$. The combined extracts were washed with brine, dried over $\mathrm{MgSO}_{4}$ and concentrated. The crude allyl alcohol was used without purification if required allyl acetate. Pure allyl alcohol could be obtained in quantative yield as a colorless viscous oil after purification by chromatography on silica gel (hexane/Et ${ }_{2} \mathrm{O}=2: 1$ to $1: 2$ ). The crude allyl alcohol was dissolved in $\mathrm{CH}_{2} \mathrm{Cl}_{2}(0.2 \mathrm{M})$ and treated with $\mathrm{Ac}_{2} \mathrm{O}$ (1.5 equiv), pyridine (1.5 equiv) and DMAP (0.05 equiv) at room temperature. After stirring for several hours, the volatiles were removed by evaporation and the residue was purified by chromatography on silica gel (hexane/Et ${ }_{2} \mathrm{O}$ as eluent) to give the corresponding allylic ester 1 . The yields of all compounds were noted in Table 3. 


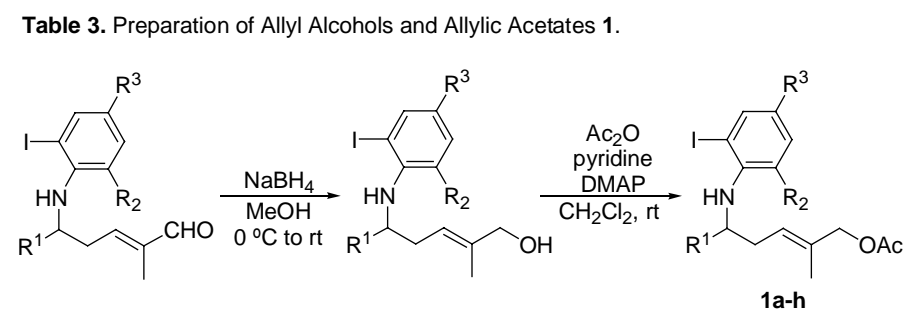

\begin{tabular}{cccccc}
\hline entry & $\mathrm{R}^{1}$ & $\mathrm{R}^{2}$ & $\mathrm{R}^{3}$ & \multicolumn{3}{c}{${\text { Yield }(\%)^{a}}^{\mathrm{a}}$} \\
\hline 1 & $\mathrm{Ph}$ & $\mathrm{H}$ & $\mathrm{H}$ & $\mathbf{a}$ & 93 \\
2 & $4-\mathrm{Me}-\mathrm{Ph}$ & $\mathrm{H}$ & $\mathrm{H}$ & $\mathbf{b}$ & 98 \\
3 & $4-\mathrm{Cl}-\mathrm{Ph}$ & $\mathrm{H}$ & $\mathrm{H}$ & $\mathbf{c}$ & 97 \\
4 & $4-\mathrm{Br}-\mathrm{Ph}$ & $\mathrm{H}$ & $\mathrm{H}$ & $\mathbf{d}$ & 92 \\
5 & $\mathrm{Ph}$ & $\mathrm{Me}$ & $\mathrm{Me}$ & $\mathbf{e}$ & 97 \\
6 & $\mathrm{Ph}$ & $\mathrm{H}$ & $\mathrm{Cl}$ & $\mathbf{f}$ & 99 \\
7 & $\mathrm{Ph}$ & $\mathrm{OMe}$ & $\mathrm{H}$ & $\mathbf{g}$ & 97 \\
8 & coO-c-Hex & $\mathrm{Me}$ & $\mathrm{Me}$ & $\mathbf{h}$ & 98 \\
\hline a Isolated yield. & & & &
\end{tabular}

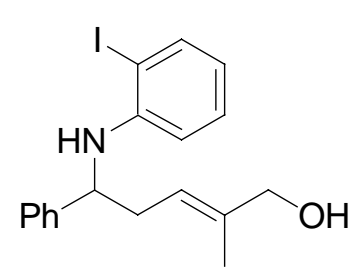

(2E)- $N$-(2-Iodophenyl)-5-amino-2-methyl-5-phenyl-2-penten-1-ol: colorless crystal, $\mathrm{mp}=64-66^{\circ} \mathrm{C}$, purified by chromatography on silica gel (hexane/Et $2 \mathrm{O}=3: 1$ to $1.5: 1) ;{ }^{1} \mathrm{H} \mathrm{NMR}\left(300 \mathrm{MHz}, \mathrm{CDCl}_{3}\right) \delta 7.62$ $(1 \mathrm{H}, \mathrm{dd}, J=8.1,1.2 \mathrm{~Hz}, \mathrm{Ar}-\mathrm{H}), 7.34-7.20(6 \mathrm{H}, \mathrm{m}, \mathrm{Ar}-\mathrm{H}), 6.98(1 \mathrm{H}, \mathrm{td}$, $J=7.5,1.2 \mathrm{~Hz}, \mathrm{Ar}-\mathrm{H}), 6.36(1 \mathrm{H}, \mathrm{td}, J=7.5,1.2 \mathrm{~Hz}, \mathrm{Ar}-\mathrm{H}), 5.47(1 \mathrm{H}$, $\mathrm{td}, J=7.2,1.2 \mathrm{~Hz}, 3-\mathrm{CH}), 4.68(1 \mathrm{H}, \mathrm{d}, J=5.1 \mathrm{~Hz}, \mathrm{NH}), 4.40(1 \mathrm{H}, \mathrm{td}, J=7.5,5.1 \mathrm{~Hz}, 5-\mathrm{CH})$, $4.03\left(2 \mathrm{H}, \mathrm{s}, 1-\mathrm{CH}_{2}\right), 2.68-2.52\left(2 \mathrm{H}, \mathrm{m}, 4-\mathrm{CH}_{2}\right), 1.74\left(3 \mathrm{H}, \mathrm{s}, 2-\mathrm{CH}_{3}\right), 1.45(1 \mathrm{H}, \mathrm{s}, \mathrm{OH}) ;{ }^{13} \mathrm{C}$ NMR $\left(75 \mathrm{MHz}, \mathrm{CDCl}_{3}\right) \delta 146.2,142.8,139.0,138.7,129.2,128.6,127.1,126.1,120.9$, 118.7, 112.1, 85.5, 68.6, 58.2, 37.1, 14.1; IR (film) 3372, 3058, 3024, 2915, 2853, 1585, 1500, 1446, 1310, 1066, 1001, 919, $739 \mathrm{~cm}^{-1}$ : HRMS-EI (m/z): $\left[\mathrm{M}^{+}\right]$calcd for $\mathrm{C}_{18} \mathrm{H}_{20} \mathrm{INO}$ 393.0590, Found: 393.0594.

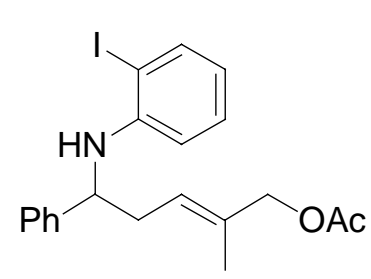

(2E)- $N$-(2-Iodophenyl)-5-amino-2-methyl-5-phenyl-2-pentenyl acetate (1a): colorless viscous oil; ${ }^{1} \mathrm{H} \mathrm{NMR}\left(300 \mathrm{MHz}, \mathrm{CDCl}_{3}\right) \delta$ $7.63(1 \mathrm{H}, \mathrm{dd}, J=7.8,1.5 \mathrm{~Hz}, \mathrm{Ar}-\mathrm{H}), 7.33-7.21$ (5H, m, Ar-H), 6.99 $(1 \mathrm{H}, \mathrm{td}, J=7.8,1.5 \mathrm{~Hz}, \mathrm{Ar}-\mathrm{H}), 6.37(1 \mathrm{H}, \mathrm{td}, J=7.8,1.5 \mathrm{~Hz}, \mathrm{Ar}-\mathrm{H})$, $6.25(1 \mathrm{H}, \mathrm{dd}, J=7.8,1.5 \mathrm{~Hz}, \mathrm{Ar}-\mathrm{H}), 5.50(1 \mathrm{H}, \mathrm{t}, J=7.5 \mathrm{~Hz}, 3-\mathrm{CH})$, $4.68(1 \mathrm{H}, \mathrm{d}, J=5.1 \mathrm{~Hz}, \mathrm{NH}), 4.49\left(2 \mathrm{H}, \mathrm{s}, 1-\mathrm{CH}_{2}\right), 4.42(1 \mathrm{H}, \mathrm{td}, J=7.5,5.1 \mathrm{~Hz}, 5-\mathrm{CH})$, 2.69-2.53 (2H, m, 4-CH $), 2.07\left(3 \mathrm{H}, \mathrm{s}, \mathrm{OCOCH}_{3}\right), 1.73\left(3 \mathrm{H}, \mathrm{s}, 2-\mathrm{CH}_{3}\right) ;{ }^{13} \mathrm{C} \mathrm{NMR}(75 \mathrm{MHz}$, $\left.\mathrm{CDCl}_{3}\right) \delta 170.8,146.1,142.5,138.8,134.1,129.2,128.6,127.1,126.1,124.0,118.7,112.1$, 85.5, 69.6, 57.9, 37.0, 21.0, 14.4; IR (film) 3386, 3058, 3024, 2928, 1738, 1585, 1504, 1446, 
1314, 1225, 1021, 1004, 919, $739 \mathrm{~cm}^{-1}$; HRMS-EI (m/z): $\left[\mathrm{M}^{+}\right]$calcd for $\mathrm{C}_{20} \mathrm{H}_{22} \mathrm{INO}_{2}$ 435.0695, Found: 435.0711.

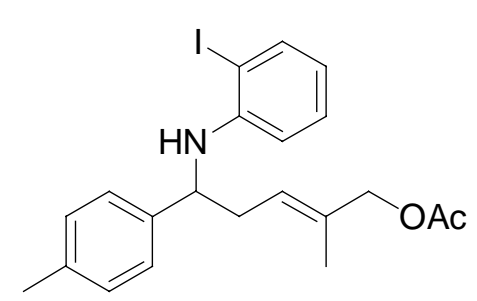

(2E)- $N$-(2-Iodophenyl)-5-amino-2-methyl-5-(4-methylphen yl)-2-pentenyl acetate (1b): colorless viscous oil; ${ }^{1} \mathrm{H}$ NMR $\left(300 \mathrm{MHz}, \mathrm{CDCl}_{3}\right) \delta 7.62(1 \mathrm{H}, \mathrm{dd}, J=7.8,1.5 \mathrm{~Hz}, \mathrm{Ar}-\mathrm{H}), 7.20$ $(2 \mathrm{H}, \mathrm{d}, J=7.8 \mathrm{~Hz}, \mathrm{Ar}-\mathrm{H}), 7.12(2 \mathrm{H}, \mathrm{d}, J=7.8 \mathrm{~Hz}, \mathrm{Ar}-\mathrm{H}), 6.99$ $(1 \mathrm{H}, \mathrm{td}, J=7.8,1.5 \mathrm{~Hz}, \mathrm{Ar}-\mathrm{H}), 6.36(1 \mathrm{H}, \mathrm{td}, J=7.8,1.5 \mathrm{~Hz}$, Ar-H), 6.27 (1H, dd, J=7.8, 1.5 Hz, Ar-H), $5.50(1 \mathrm{H}, \mathrm{td}, J=7.5,1.2 \mathrm{~Hz}, 3-\mathrm{CH}), 4.66(1 \mathrm{H}, \mathrm{d}$, $J=5.4 \mathrm{~Hz}, \mathrm{NH}), 4.49\left(2 \mathrm{H}, \mathrm{s}, 1-\mathrm{CH}_{2}\right), 4.39(1 \mathrm{H}, \mathrm{td}, J=7.2,5.4 \mathrm{~Hz}, 5-\mathrm{CH}), 2.67-2.51(2 \mathrm{H}, \mathrm{m}$, 4- $\left.\mathrm{CH}_{2}\right), 2.32\left(3 \mathrm{H}, \mathrm{s}, \mathrm{Ar}-\mathrm{CH}_{3}\right), 2.07\left(3 \mathrm{H}, \mathrm{s}, \mathrm{OCOCH}_{3}\right), 1.73\left(3 \mathrm{H}, \mathrm{s}, 2-\mathrm{CH}_{3}\right) ;{ }^{13} \mathrm{C} \mathrm{NMR}(75$ $\left.\mathrm{MHz}, \mathrm{CDCl}_{3}\right) \delta 170.8,146.3,139.6,139(\mathrm{~m}), 136.7,134.0,129(\mathrm{~m}), 126(\mathrm{~m}), 124.34,124.28$, $119(\mathrm{~m}), 112(\mathrm{~m}), 85.5,69.7(\mathrm{t}, J=4 \mathrm{~Hz}), 57.7,37.1,21(\mathrm{~m}), 14.4$; IR (film) 3379, 3010, 2976, 2915, 2860, 1738, 1585, 1504, 1450, 1314, 1225, 1018, 1001, 814, $743 \mathrm{~cm}^{-1}$; HRMS-EI $(\mathrm{m} / \mathrm{z}):\left[\mathrm{M}^{+}\right]$calcd for $\mathrm{C}_{21} \mathrm{H}_{24} \mathrm{INO}_{2}$ (449.0852, Found: 449.0843 .

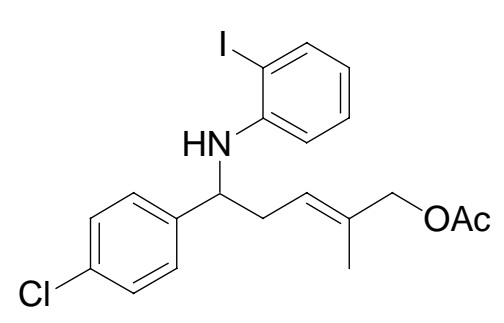

(2E)-N-(2-Iodophenyl)-5-amino-2-methyl-5-(4-chlorophe nyl)-2-pentenyl acetate (1c): colorless viscous oil; ${ }^{1} \mathrm{H}$ NMR $\left(300 \mathrm{MHz}, \mathrm{CDCl}_{3}\right) \delta 7.64(1 \mathrm{H}, \mathrm{dd}, J=7.8,1.5 \mathrm{~Hz}, \mathrm{Ar}-\mathrm{H})$, 7.31-7.23 (4H, m, Ar-H), $7.00(1 \mathrm{H}, \mathrm{td}, J=7.8,1.5 \mathrm{~Hz}, \mathrm{Ar}-\mathrm{H})$, $6.39(1 \mathrm{H}, \mathrm{td}, J=7.8,1.5 \mathrm{~Hz}, \mathrm{Ar}-\mathrm{H}), 6.19(1 \mathrm{H}, \mathrm{dd}, J=7.8,1.5$ $\mathrm{Hz}, \mathrm{Ar}-\mathrm{H}), 5.47$ (1H, td, $J=7.5,1.5 \mathrm{~Hz}, 3-\mathrm{CH}), 4.66(1 \mathrm{H}, \mathrm{d}, J$ $=5.1 \mathrm{~Hz}, \mathrm{NH}), 4.49\left(2 \mathrm{H}, \mathrm{s}, 1-\mathrm{CH}_{2}\right), 4.39(1 \mathrm{H}, \mathrm{td}, J=7.2,5.1 \mathrm{~Hz}, 5-\mathrm{CH}), 2.67-2.50(2 \mathrm{H}, \mathrm{m}$, 4- $\left.\mathrm{CH}_{2}\right), 2.07\left(3 \mathrm{H}, \mathrm{s}, \mathrm{OCOCH}_{3}\right), 1.72\left(3 \mathrm{H}, \mathrm{s}, 2-\mathrm{CH}_{3}\right) ;{ }^{13} \mathrm{C} \mathrm{NMR}\left(75 \mathrm{MHz}, \mathrm{CDCl}_{3}\right) \delta 170.8$, $145.9,141.2$, 138.9, 134.6, 132.8, 129.2, 128.8, 127.6, 123.5, 119.0, 112.1, 85.6, 69.5, 57.5, 36.9, 21.0, 14.4; IR (film) 3379, 3058, 2976, 2922, 1735, 1585, 1500, 1450, 1314, 1225, 1086, 1004, 824, $743 \mathrm{~cm}^{-1}$; HRMS-EI (m/z): [ $\left.\mathrm{M}^{+}\right]$calcd for $\mathrm{C}_{20} \mathrm{H}_{21} \mathrm{INO}_{2}$ 469.0306, Found: 469.0313.

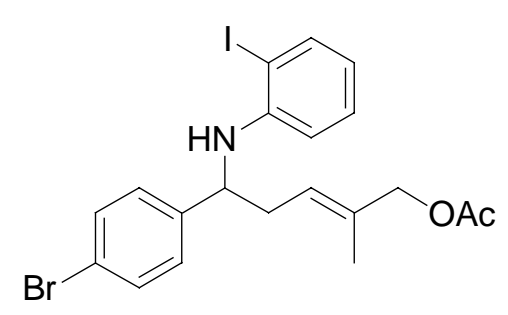

(2E)- $N$-(2-Iodophenyl)-5-amino-2-methyl-5-(4-bromophe nyl)-2-pentenyl acetate (1d): colorless viscous oil; ${ }^{1} \mathrm{H}$ NMR $\left(300 \mathrm{MHz}, \mathrm{CDCl}_{3}\right) \delta 7.64(1 \mathrm{H}, \mathrm{dd}, J=7.8,1.5 \mathrm{~Hz}, \mathrm{Ar}-\mathrm{H})$, $7.44(2 \mathrm{H}, \mathrm{dt}, J=8.1,1.8 \mathrm{~Hz}, \mathrm{Ar}-\mathrm{H}), 7.20(2 \mathrm{H}, \mathrm{dt}, J=8.1,1.8$ $\mathrm{Hz}, \mathrm{Ar}-\mathrm{H}), 7.00(1 \mathrm{H}, \mathrm{td}, J=7.8,1.5 \mathrm{~Hz}, \mathrm{Ar}-\mathrm{H}), 6.39(1 \mathrm{H}, \mathrm{td}, J$ $=7.8,1.5 \mathrm{~Hz}, \mathrm{Ar}-\mathrm{H}), 6.19(1 \mathrm{H}, \mathrm{dd}, J=7.8,1.5 \mathrm{~Hz}, \mathrm{Ar}-\mathrm{H})$, $5.47(1 \mathrm{H}, \mathrm{td}, J=7.5,0.9 \mathrm{~Hz}, 3-\mathrm{CH}), 4.66(1 \mathrm{H}, \mathrm{d}, J=5.1 \mathrm{~Hz}, \mathrm{NH}), 4.49\left(2 \mathrm{H}, \mathrm{s}, 1-\mathrm{CH}_{2}\right), 4.37$ 
$(1 \mathrm{H}, \mathrm{td}, J=7.2,5.1 \mathrm{~Hz}, 5-\mathrm{CH}), 2.66-2.50\left(2 \mathrm{H}, \mathrm{m}, 4-\mathrm{CH}_{2}\right), 2.07\left(3 \mathrm{H}, \mathrm{s}, \mathrm{OCOCH}_{3}\right), 1.73(3 \mathrm{H}$, s, 2- $\left.\mathrm{CH}_{3}\right) ;{ }^{13} \mathrm{C} \mathrm{NMR}\left(75 \mathrm{MHz}, \mathrm{CDCl}_{3}\right) \delta 170.8,145.8,141.7,138.9,134.6,131.7,129.2$, 128.0, 123.5, 120.8, 119.0, 112.1, 85.6, 69.5, 57.5, 36.9, 21.0, 14.4; IR (film) 3379, 3058, 2922, 1735, 1585, 1500, 1453, 1314, 1225, 1072, 1008, 821, $743 \mathrm{~cm}^{-1}$; HRMS-EI (m/z): $\left[\mathrm{M}^{+}\right]$calcd for $\mathrm{C}_{20} \mathrm{H}_{21} \mathrm{BrINO}_{2}$ 512.9800, Found: 512.9795 .

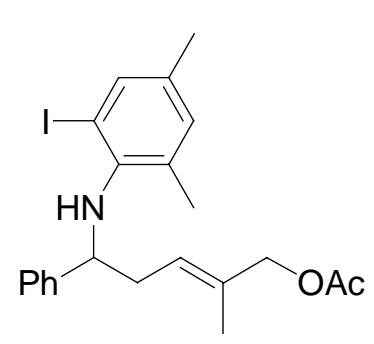

(2E)-N-(2,4-Dimethyl-6-iodophenyl)-5-amino-2-methyl-5-phenyl -2-pentenyl acetate (1e): colorless viscous oil; ${ }^{1} \mathrm{H}$ NMR $(300 \mathrm{MHz}$, $\left.\mathrm{CDCl}_{3}\right) \delta 7.39(1 \mathrm{H}, \mathrm{d}, J=1.2 \mathrm{~Hz}, \mathrm{Ar}-\mathrm{H}), 7.31-7.18(5 \mathrm{H}, \mathrm{m}, \mathrm{Ar}-\mathrm{H})$, $6.81(1 \mathrm{H}, \mathrm{d}, J=1.2 \mathrm{~Hz}, \mathrm{Ar}-\mathrm{H}), 5.40(1 \mathrm{H}, \mathrm{td}, J=7.2,0.9 \mathrm{~Hz}, 3-\mathrm{CH})$, 4.42-4.34 $(1 \mathrm{H}, \mathrm{m}, 5-\mathrm{CH}), 4.38\left(2 \mathrm{H}, \mathrm{s}, 1-\mathrm{CH}_{2}\right), 3.82(1 \mathrm{H}, \mathrm{s}, \mathrm{NH})$, 2.72-2.56 $\left(2 \mathrm{H}, \mathrm{m}, 4-\mathrm{CH}_{2}\right), 2.18\left(3 \mathrm{H}, \mathrm{s}, \mathrm{Ar}-\mathrm{CH}_{3}\right), 2.16(3 \mathrm{H}, \mathrm{s}$, $\left.\mathrm{Ar}-\mathrm{CH}_{3}\right), 2.02\left(3 \mathrm{H}, \mathrm{s}, \mathrm{OCOCH}_{3}\right), 1.58\left(3 \mathrm{H}, \mathrm{s}, 2-\mathrm{CH}_{3}\right) ;{ }^{13} \mathrm{C} \mathrm{NMR}\left(75 \mathrm{MHz}, \mathrm{CDCl}_{3}\right) \delta 170.8$, $143.8,142.7,137.3,132.8,132.7,132.4,129.7,128.3,127.13,127.11,125.4,95.4,69.8$, 61.7, 35.4, 20.9, 20.3, 19.9, 14.2; IR (film) 3338, 3024, 2922, 2853, 1735, 1470, 1439, 1371, 1225, 1021, 848, $753 \mathrm{~cm}^{-1}$; HRMS-EI (m/z): $\left[\mathrm{M}^{+}\right]$calcd for $\mathrm{C}_{22} \mathrm{H}_{26} \mathrm{INO}_{2} 464.1087$, Found: 464.1093.

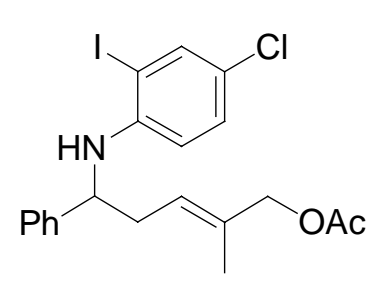

(2E)- $N$-(4-Chloro-2-Iodophenyl)-5-amino-2-methyl-5-phenyl-2-p entenyl acetate (1f): colorless viscous oil; ${ }^{1} \mathrm{H}$ NMR $(300 \mathrm{MHz}$, $\left.\mathrm{CDCl}_{3}\right) \delta 7.60(1 \mathrm{H}, \mathrm{d}, J=2.4 \mathrm{~Hz}, \mathrm{Ar}-\mathrm{H}), 7.35-7.21(5 \mathrm{H}, \mathrm{m}, \mathrm{Ar}-\mathrm{H})$, $6.95(1 \mathrm{H}, \mathrm{dd}, J=8.7,2.4 \mathrm{~Hz}, \mathrm{Ar}-\mathrm{H}), 6.14(1 \mathrm{H}, \mathrm{d}, J=8.7 \mathrm{~Hz}, \mathrm{Ar}-\mathrm{H})$, $5.48(1 \mathrm{H}, \mathrm{td}, J=7.5,1.2 \mathrm{~Hz}, 3-\mathrm{CH}), 4.67(1 \mathrm{H}, \mathrm{d}, J=5.4 \mathrm{~Hz}, \mathrm{NH})$, $4.49\left(2 \mathrm{H}, \mathrm{s}, 1-\mathrm{CH}_{2}\right), 4.38(1 \mathrm{H}, \mathrm{td}, J=7.2,5.4 \mathrm{~Hz}, 5-\mathrm{CH}), 2.69-2.52\left(2 \mathrm{H}, \mathrm{m}, 4-\mathrm{CH}_{2}\right), 2.07(3 \mathrm{H}$, s, $\left.\mathrm{OCOCH}_{3}\right), 1.72\left(3 \mathrm{H}, \mathrm{s}, 2-\mathrm{CH}_{3}\right) ;{ }^{13} \mathrm{C} \mathrm{NMR}\left(75 \mathrm{MHz}, \mathrm{CDCl}_{3}\right) \delta 170.8,144.9,142.1,137.7$, 134.4, 129.0, 128.7, 127.3, 126.1, 123.7, 122.1, 112.3, 84.9, 69.5, 58.1, 37.0, 21.0, 14.4; IR (film) 3372, 3058, 3024, 2922, 1735, 1582, 1490, 1450, 1385, 1310, 1225, 1021, 868, 797, $753 \mathrm{~cm}^{-1}$; HRMS-ESI (m/z): $[\mathrm{M}+\mathrm{H}]^{+}$calcd for $\mathrm{C}_{20} \mathrm{H}_{22} \mathrm{INO}_{2} 470.0378$, Found: 470.0385 .

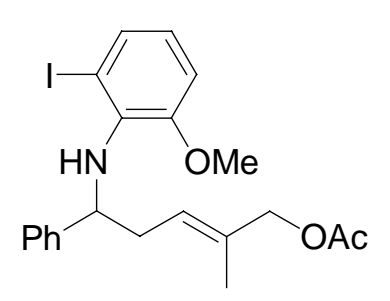

(2E)-N-(2-Iodo-6-methoxyphenyl)-5-amino-2-methyl-5-phenyl-2 -pentenyl acetate (1g): colorless viscous oil; ${ }^{1} \mathrm{H}$ NMR $(300 \mathrm{MHz}$, $\left.\mathrm{CDCl}_{3}\right) \delta$ 7.30-7.12 $(6 \mathrm{H}, \mathrm{m}, \mathrm{Ar}-\mathrm{H}), 6.66(1 \mathrm{H}, \mathrm{dd}, J=8.1,1.2 \mathrm{~Hz}$, Ar-H), $6.48(1 \mathrm{H}, \mathrm{t}, J=8.1 \mathrm{~Hz}, \mathrm{Ar}-\mathrm{H}), 5.47(1 \mathrm{H}, \mathrm{td}, J=7.2,1.2 \mathrm{~Hz}$, 3-CH), $4.97(1 \mathrm{H}, \mathrm{br}, \mathrm{NH}), 4.42\left(2 \mathrm{H}, \mathrm{s}, 1-\mathrm{CH}_{2}\right), 4.24(1 \mathrm{H}, \mathrm{br}, 5-\mathrm{CH})$, $3.67\left(3 \mathrm{H}, \mathrm{s}, \mathrm{Ar}-\mathrm{OCH}_{3}\right), 2.68-2.53\left(2 \mathrm{H}, \mathrm{m}, 4-\mathrm{CH}_{2}\right), 2.03\left(3 \mathrm{H}, \mathrm{s}, \mathrm{OCOCH}_{3}\right), 1.61(3 \mathrm{H}, \mathrm{s}$, $\left.2-\mathrm{CH}_{3}\right) ;{ }^{13} \mathrm{C} \mathrm{NMR}\left(75 \mathrm{MHz}, \mathrm{CDCl}_{3}\right) \delta 170.9,150.2,143.2,137.7,132.5,131.5,128.0,126.9$, 
126.8, 125.3, 122.1, 111.8, 91.5, 69.9, 60.4, 55.6, 35.9, 21.0, 14.2; IR (film) 3338, 3058, 3024, 2935, 2826, 1735, 1568, 1463, 1371, 1225, 1025, 851, $753 \mathrm{~cm}^{-1}$; HRMS-ESI (m/z): $[\mathrm{M}+\mathrm{H}]^{+}$calcd for $\mathrm{C}_{21} \mathrm{H}_{25} \mathrm{INO}_{2} 466.0873$, Found: 466.0875 .

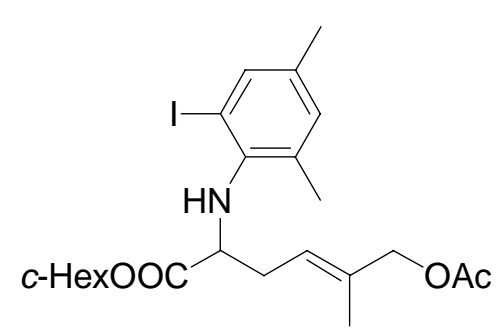

(2E)- $N$-(2,4-Dimethyl-6-iodophenyl)-5-amino-5-cyclohex ylcarbonyl-2-methyl-2-pentenyl acetate (1h): colorless viscous oil; ${ }^{1} \mathrm{H}$ NMR $\left(300 \mathrm{MHz}, \mathrm{CDCl}_{3}\right) \delta 7.43(1 \mathrm{H}, \mathrm{s}, \mathrm{Ar}-\mathrm{H})$, $6.87(1 \mathrm{H}, \mathrm{s}, \operatorname{Ar}-\mathrm{H}), 5.46(1 \mathrm{H}, \mathrm{td}, J=7.2,1.2 \mathrm{~Hz}, 3-\mathrm{CH})$, 4.80-4.71 (1H, m, OCH), $4.44\left(2 \mathrm{H}, \mathrm{s}, 1-\mathrm{CH}_{2}\right), 4.20-4.11(2 \mathrm{H}$, $\mathrm{m}, \mathrm{NH}$ and $5-\mathrm{CH}), 2.62-2.47\left(2 \mathrm{H}, \mathrm{m}, 4-\mathrm{CH}_{2}\right), 2.28(3 \mathrm{H}, \mathrm{s}$, $\left.\mathrm{Ar}-\mathrm{CH}_{3}\right), 2.18\left(3 \mathrm{H}, \mathrm{s}, \mathrm{Ar}-\mathrm{CH}_{3}\right), 2.06\left(3 \mathrm{H}, \mathrm{s}, \mathrm{OCOCH}_{3}\right), 1.81-1.47(5 \mathrm{H}, \mathrm{m}), 1.60(3 \mathrm{H}, \mathrm{s}$, 2- $\left.\mathrm{CH}_{3}\right), 1.42-1.20(5 \mathrm{H}, \mathrm{m}) ;{ }^{13} \mathrm{C} \mathrm{NMR}\left(75 \mathrm{MHz}, \mathrm{CDCl}_{3}\right) \delta 172.7,170.8,143.2,137.7,133.4$, 133.1, 132.6, 129.4, 123.2, 94.0, 73.6, 69.7, 59.3, 31.6, 31.48, 31.45, 25.3, 23.6, 20.9, 19.9, 14.1; IR (film) 3324, 2928, 2853, 1732, 1470, 1446, 1375, 1225, 1120, 1015, 851, $783 \mathrm{~cm}^{-1}$; HRMS-ESI (m/z): $[\mathrm{M}+\mathrm{H}]^{+}$calcd for $\mathrm{C}_{23} \mathrm{H}_{33} \mathrm{INO}_{4}$ 514.1448, Found: 514.1428.

General procedure of palladium catalyzed cross-coupling reactions between an aryl iodide and allyl acetate: To a mixture of 1 (1.0 equiv), (o-tolyl $)_{3} \mathrm{P}(22 \mathrm{~mol} \%)$ and $n$ - $\mathrm{BuNMe}_{2}$ (2.0 equiv) in $\mathrm{CH}_{3} \mathrm{CN}-\mathrm{H}_{2} \mathrm{O}(10: 1,0.1 \mathrm{M})$ was added $\mathrm{Pd}_{2}(\mathrm{dba})_{3} \cdot \mathrm{CHCl}_{3}(5 \mathrm{~mol} \%)$. The mixture was degassed and filled with a $\mathrm{N}_{2}$ gas quickly and refluxed for $6 \mathrm{~h}$ under a $\mathrm{N}_{2}$ atmosphere. The resulting mixture was cooled to room temperature and treated with saturated $\mathrm{NH}_{4} \mathrm{Cl}$. Extractive workup and purification of the residue by column chromatography on silica gel (hexane/ $\mathrm{Et}_{2} \mathrm{O}=40: 1$ to 20:1 as eluent) gave 2,4-disubustituted-1,2,3,4-tetrahydroquinoline 2 . The yields of all compounds are noted in Table 4. 

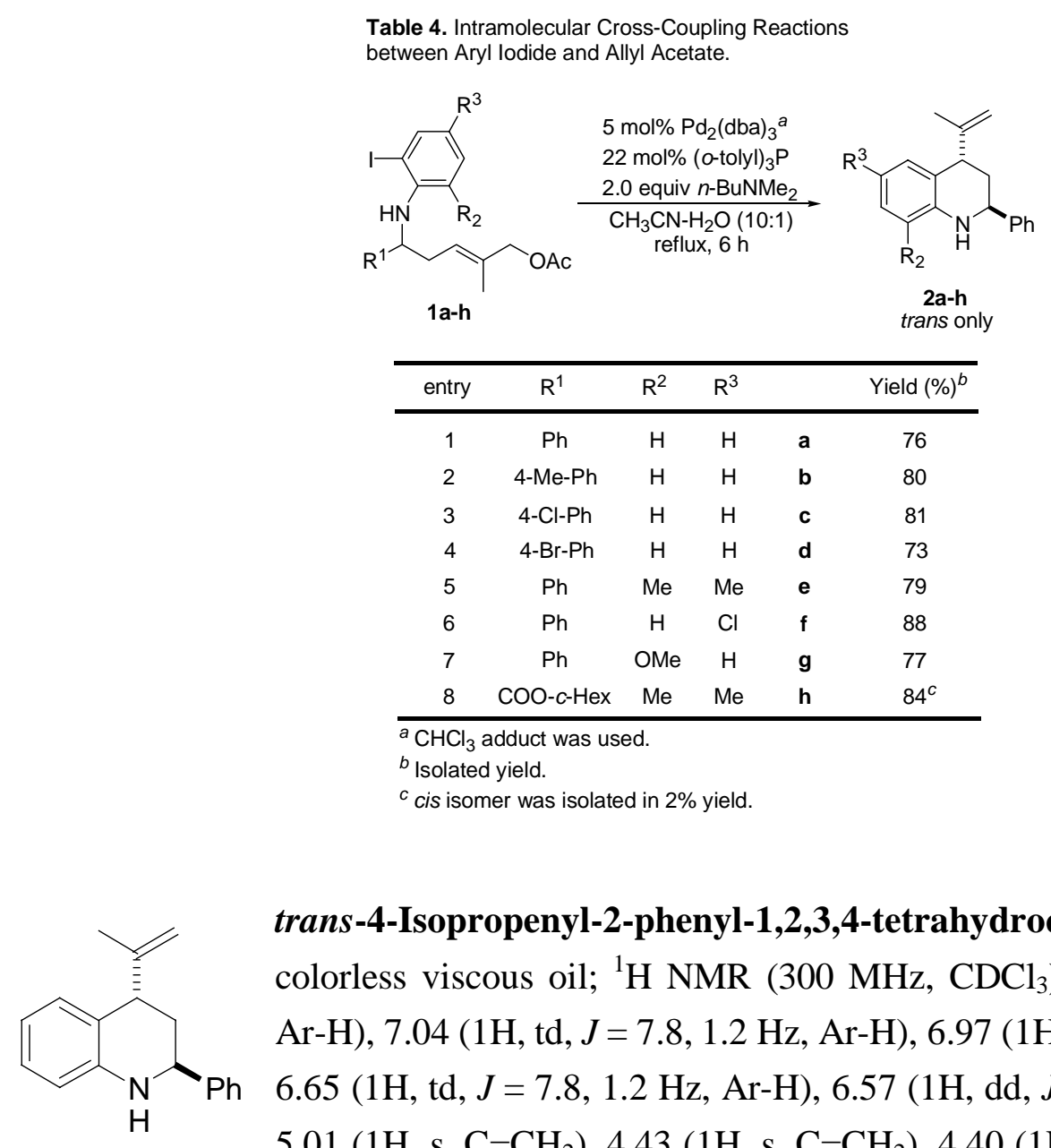

trans-4-Isopropenyl-2-phenyl-1,2,3,4-tetrahydroquinoline (2a):

colorless viscous oil; ${ }^{1} \mathrm{H}$ NMR $\left(300 \mathrm{MHz}, \mathrm{CDCl}_{3}\right) \delta$ 7.40-7.26 $(5 \mathrm{H}, \mathrm{m}$, Ar-H), $7.04(1 \mathrm{H}, \mathrm{td}, J=7.8,1.2 \mathrm{~Hz}, \mathrm{Ar}-\mathrm{H}), 6.97(1 \mathrm{H}, \mathrm{d}, J=7.8 \mathrm{~Hz}, \mathrm{Ar}-\mathrm{H})$,

$6.65(1 \mathrm{H}, \mathrm{td}, J=7.8,1.2 \mathrm{~Hz}, \mathrm{Ar}-\mathrm{H}), 6.57(1 \mathrm{H}, \mathrm{dd}, J=7.8,1.2 \mathrm{~Hz}, \mathrm{Ar}-\mathrm{H})$, $5.01\left(1 \mathrm{H}, \mathrm{s}, \mathrm{C}=\mathrm{CH}_{2}\right), 4.43\left(1 \mathrm{H}, \mathrm{s}, \mathrm{C}=\mathrm{CH}_{2}\right), 4.40(1 \mathrm{H}, \mathrm{dd}, J=9.6,3.3 \mathrm{~Hz}$,

2-CH), $4.07(1 \mathrm{H}, \mathrm{s}, \mathrm{NH}), 3.41(1 \mathrm{H}, \mathrm{dd}, J=5.4,3.6 \mathrm{~Hz}, 4-\mathrm{CH}), 2.11(1 \mathrm{H}, \mathrm{ddd}, J=13.2$, 3.6, $\left.3.3 \mathrm{~Hz}, 3-\mathrm{CH}_{2}\right), 2.00\left(1 \mathrm{H}\right.$, ddd, $\left.J=13.2,9.6,5.4 \mathrm{~Hz}, 3-\mathrm{CH}_{2}\right), 1.83\left(3 \mathrm{H}, \mathrm{s}, \mathrm{CH}_{3}\right) ;{ }^{13} \mathrm{C} \mathrm{NMR}(75$ $\left.\mathrm{MHz}, \mathrm{CDCl}_{3}\right) \delta 149.1,144.62,144.57,130.3,128.6,127.4,127.3,126.7,122.0,117.0,114.7$, 113.9, 52.4, 43.4, 34.2, 21.1; IR (film) 3392, 3024, 2956, 2915, 2853, 1606, 1483, 1314, $1256,1113,1025,896,746 \mathrm{~cm}^{-1}$; HRMS-EI (m/z): $\left[\mathrm{M}^{+}\right]$calcd for $\mathrm{C}_{18} \mathrm{H}_{19} \mathrm{~N} 249.1518$, Found: 249.1519 .

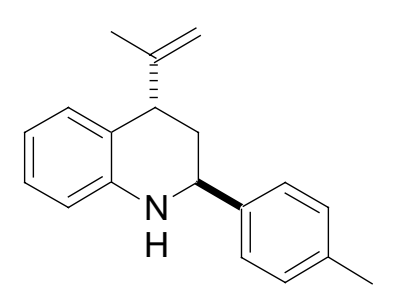

trans-4-Isopropenyl-2-(4-methylphenyl)-1,2,3,4-tetrahydroquin oline (2b): colorless viscous oil; ${ }^{1} \mathrm{H}$ NMR $\left(300 \mathrm{MHz}, \mathrm{CDCl}_{3}\right) \delta 7.27$ $(2 \mathrm{H}, \mathrm{d}, J=8.1 \mathrm{~Hz}, \mathrm{Ar}-\mathrm{H}), 7.15(2 \mathrm{H}, \mathrm{d}, J=8.1 \mathrm{~Hz}, \mathrm{Ar}-\mathrm{H}), 7.03(1 \mathrm{H}$, $\operatorname{td}, J=7.5,1.2 \mathrm{~Hz}, \mathrm{Ar}-\mathrm{H}), 6.96(1 \mathrm{H}, \mathrm{d}, J=7.5 \mathrm{~Hz}, \mathrm{Ar}-\mathrm{H}), 6.64(1 \mathrm{H}$, td, $J=7.5,1.2 \mathrm{~Hz}, \mathrm{Ar}-\mathrm{H}), 6.55(1 \mathrm{H}, \mathrm{d}, J=7.5 \mathrm{~Hz}, \mathrm{Ar}-\mathrm{H}), 5.00(1 \mathrm{H}, \mathrm{s}$, $\left.\mathrm{C}=\mathrm{CH}_{2}\right), 4.42\left(1 \mathrm{H}, \mathrm{d}, J=0.9 \mathrm{~Hz}, \mathrm{C}=\mathrm{CH}_{2}\right), 4.35(1 \mathrm{H}, \mathrm{dd}, J=9.9,3.3 \mathrm{~Hz}, 4-\mathrm{CH}), 4.02(1 \mathrm{H}, \mathrm{s}$, 
$\mathrm{NH}), 3.41(1 \mathrm{H}, \mathrm{dd}, J=5.7,3.6 \mathrm{~Hz}, 2-\mathrm{CH}), 2.35\left(3 \mathrm{H}, \mathrm{s}, \mathrm{Ar}-\mathrm{CH}_{3}\right), 2.08(1 \mathrm{H}, \mathrm{ddd}, J=13.2,3.6$, $\left.3.3 \mathrm{~Hz}, 3-\mathrm{CH}_{2}\right), 1.98\left(1 \mathrm{H}, \mathrm{ddd}, J=13.2,9.9,5.7 \mathrm{~Hz}, 3-\mathrm{CH}_{2}\right), 1.83\left(3 \mathrm{H}, \mathrm{d}, J=0.9 \mathrm{~Hz}, \mathrm{CH}_{3}\right)$; ${ }^{13} \mathrm{C} \mathrm{NMR}\left(75 \mathrm{MHz}, \mathrm{CDCl}_{3}\right) \delta 149.1,144.7,141.6,137.1,130.3,129.2,127.3,126.6,122.0$, 116.9, 114.7, 113.9, 52.0, 43.4, 34.2, 21.2, 21.1; IR (film) 3392, 3010, 2922, 2853, 1606,

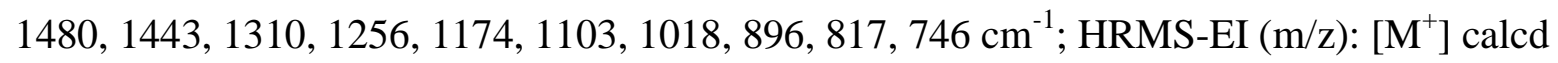
for $\mathrm{C}_{19} \mathrm{H}_{21} \mathrm{~N} 263.1674$, Found: 263.1679 .

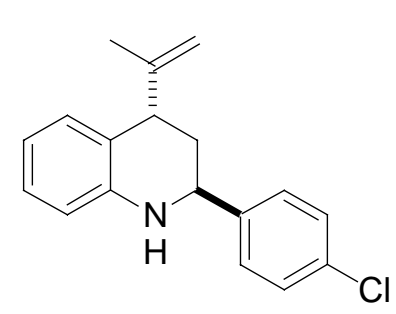

trans-2-(4-Chlorophenyl)-4-isopropenyl-1,2,3,4-tetrahydroquin oline (2c): colorless viscous oil; ${ }^{1} \mathrm{H}$ NMR (300 MHz, $\left.\mathrm{CDCl}_{3}\right) \delta 7.31$ (4H, s, Ar-H), $7.04(1 \mathrm{H}, \mathrm{t}, J=7.8 \mathrm{~Hz}, \mathrm{Ar}-\mathrm{H}), 6.97(1 \mathrm{H}, \mathrm{d}, J=7.8 \mathrm{~Hz}$, Ar-H), 6.67 (1H, t, $J=7.8 \mathrm{~Hz}, \mathrm{Ar}-\mathrm{H}), 6.57$ (1H, d, $J=7.8 \mathrm{~Hz}, \mathrm{Ar}-\mathrm{H})$, $5.01\left(1 \mathrm{H}, \mathrm{s}, \mathrm{C}=\mathrm{CH}_{2}\right), 4.42\left(1 \mathrm{H}, \mathrm{s}, \mathrm{C}=\mathrm{CH}_{2}\right), 4.38(1 \mathrm{H}, \mathrm{dd}, J=9.6,3.3$ $\mathrm{Hz}, 2-\mathrm{CH}), 4.03(1 \mathrm{H}, \mathrm{s}, \mathrm{NH}), 3.39(1 \mathrm{H}, \mathrm{dd}, J=5.4,3.9 \mathrm{~Hz}, 4-\mathrm{CH})$, $2.07\left(1 \mathrm{H}\right.$, ddd, $\left.J=13.2,3.9,3.3 \mathrm{~Hz}, 3-\mathrm{CH}_{2}\right), 1.95\left(1 \mathrm{H}\right.$, ddd, $\left.J=13.2,9.6,5.4 \mathrm{~Hz}, 3-\mathrm{CH}_{2}\right)$, $1.82\left(3 \mathrm{H}, \mathrm{s}, \mathrm{CH}_{3}\right) ;{ }^{13} \mathrm{C}$ NMR $\left(75 \mathrm{MHz}, \mathrm{CDCl}_{3}\right) \delta 148.9,144.3,143.1,133.0,130.2,128.7$, 128.0, 127.4, 122.0, 117.2, 114.9, 114.0, 51.8, 43.2, 34.3, 21.1; IR (film) 3392, 3044, 3010, 2922, 2853, 1602, 1483, 1310, 1252, 1089, 1011, 902, 824, $743 \mathrm{~cm}^{-1}$; HRMS-EI (m/z): [M $\left.{ }^{+}\right]$ calcd for $\mathrm{C}_{18} \mathrm{H}_{18} \mathrm{~N} 283.1123$, Found: 283.1124.

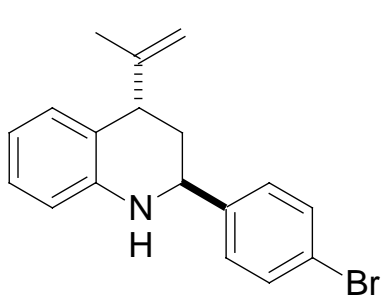

trans-2-(4-Bromophenyl)-4-isopropenyl-1,2,3,4-tetrahydroquin oline (2d): colorless viscous oil; ${ }^{1} \mathrm{H} \mathrm{NMR}\left(300 \mathrm{MHz}, \mathrm{CDCl}_{3}\right) \delta$ $7.46(2 \mathrm{H}, \mathrm{dt}, J=8.4,2.4 \mathrm{~Hz}, \mathrm{Ar}-\mathrm{H}), 7.25(2 \mathrm{H}, \mathrm{dt}, J=8.4,2.4 \mathrm{~Hz}$, Ar-H), $7.04(1 \mathrm{H}, \mathrm{td}, J=7.5,1.2 \mathrm{~Hz}, \mathrm{Ar}-\mathrm{H}), 6.96(1 \mathrm{H}, \mathrm{dd}, J=7.5$, $1.2 \mathrm{~Hz}, \mathrm{Ar}-\mathrm{H}), 6.67(1 \mathrm{H}, \mathrm{td}, J=7.5,1.2 \mathrm{~Hz}, \mathrm{Ar}-\mathrm{H}), 6.57(1 \mathrm{H}, \mathrm{dd}, J$ = 7.5, $1.2 \mathrm{~Hz}, \mathrm{Ar}-\mathrm{H}), 5.01\left(1 \mathrm{H}, \mathrm{s}, \mathrm{C}=\mathrm{CH}_{2}\right), 4.41\left(1 \mathrm{H}, \mathrm{s}, \mathrm{C}=\mathrm{CH}_{2}\right)$, $4.37(1 \mathrm{H}, \mathrm{dd}, J=9.6,3.3 \mathrm{~Hz}, 2-\mathrm{CH}), 4.03(1 \mathrm{H}, \mathrm{s}, \mathrm{NH}), 3.38(1 \mathrm{H}, \mathrm{dd}, J=5.4,3.9 \mathrm{~Hz}, 4-\mathrm{CH})$, 2.07 (1H, ddd, $\left.J=13.2,3.9,3.3 \mathrm{~Hz}, 3-\mathrm{CH}_{2}\right), 1.94\left(1 \mathrm{H}, \mathrm{ddd}, J=13.2,9.6,5.4 \mathrm{~Hz}, 3-\mathrm{CH}_{2}\right)$, $1.82\left(3 \mathrm{H}, \mathrm{s}, \mathrm{CH}_{3}\right) ;{ }^{13} \mathrm{C} \mathrm{NMR}\left(75 \mathrm{MHz}, \mathrm{CDCl}_{3}\right) \delta 148.8,144,3,143.6,132(\mathrm{~m}), 131(\mathrm{~m}), 130$ (m), 129 (m), 128 (m), 127 (m), 121.9, 121.0, 117 (m), 115.1, 114.7, 114.1, 113.9, 51.8 (d, J $=6 \mathrm{~Hz}), 43.1(\mathrm{~d}, J=10 \mathrm{~Hz}), 34.2(\mathrm{t}, J=10 \mathrm{~Hz}), 21.1$; IR (film) 3392, 3044, 2956, 2922, 2853, 1602, 1483, 1314, 1256, 1117, 1069, 1004, 899, 821, $746 \mathrm{~cm}^{-1}$; HRMS-EI (m/z): [M+] calcd for $\mathrm{C}_{18} \mathrm{H}_{18} \mathrm{BrN}$ 327.0623, Found: 327.0614 .

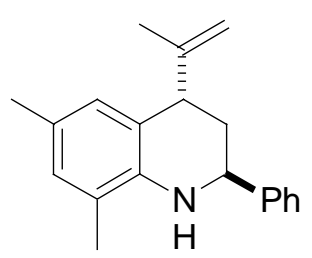

trans-6,8-Dimethyl-4-isopropenyl-2-phenyl-1,2,3,4-tetrahydroquino line (2e): colorless viscous oil; ${ }^{1} \mathrm{H}$ NMR $\left(300 \mathrm{MHz}, \mathrm{CDCl}_{3}\right) \delta 7.42-7.25$ $(5 \mathrm{H}, \mathrm{m}, \mathrm{Ar}-\mathrm{H}), 6.79$ (1H, s, Ar-H), $6.69(1 \mathrm{H}, \mathrm{s}, \mathrm{Ar}-\mathrm{H}), 5.01(1 \mathrm{H}, \mathrm{s}$, 
$\left.\mathrm{C}=\mathrm{CH}_{2}\right), 4.44-4.39(1 \mathrm{H}, \mathrm{m}, 2-\mathrm{CH}), 4.42\left(1 \mathrm{H}, \mathrm{s}, \mathrm{C}=\mathrm{CH}_{2}\right), 3.77(1 \mathrm{H}, \mathrm{s}, \mathrm{NH}), 3.39(1 \mathrm{H}, \mathrm{dd}, J=$ 5.4, 3.6 Hz, 4-CH), $2.21\left(3 \mathrm{H}, \mathrm{s}, \mathrm{Ar}-\mathrm{CH}_{3}\right), 2.12-2.06\left(1 \mathrm{H}, \mathrm{m}, 3-\mathrm{CH}_{2}\right), 2.10\left(3 \mathrm{H}, \mathrm{s}, \mathrm{Ar}-\mathrm{CH}_{3}\right)$, $1.98\left(1 \mathrm{H}, \mathrm{ddd}, J=13.2,9.9,5.4 \mathrm{~Hz}, 3-\mathrm{CH}_{2}\right), 1.84\left(3 \mathrm{H}, \mathrm{s}, \mathrm{CH}_{3}\right) ;{ }^{13} \mathrm{C} \mathrm{NMR}\left(75 \mathrm{MHz}, \mathrm{CDCl}_{3}\right)$ $\delta 149.3,145.1,140.3,129.2,128.6,128.5,128.5$ (d, $J=8 \mathrm{~Hz}), 127.4,126.7,125.4,121.5$, 120.9, 114.6 (d, $J=8 \mathrm{~Hz}), 52.6,43.7$ (d, $J=3 \mathrm{~Hz}), 34.4,21.2,20.4$ (d, $J=3 \mathrm{~Hz}), 17.3$; IR (film) 3413, 3031, 2962, 2915, 2853, 1643, 1487, 1450, 1310, 1259, 1062, 1028, 896, 858, $756 \mathrm{~cm}^{-1}$; HRMS-EI (m/z): [ $\left.\mathrm{M}^{+}\right]$calcd for $\mathrm{C}_{20} \mathrm{H}_{23} \mathrm{~N}$ 277.1831, Found: 277.1832.

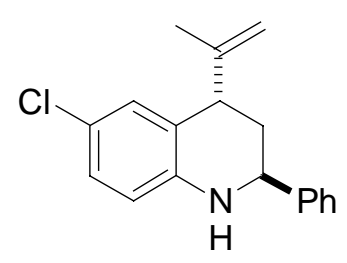

trans-6-Chloro-4-isopropenyl-2-phenyl-1,2,3,4-tetrahydroquinoline (2f): colorless viscous oil; ${ }^{1} \mathrm{H} \mathrm{NMR}\left(300 \mathrm{MHz}, \mathrm{CDCl}_{3}\right) \delta$ 7.36-7.26 (5H, m, Ar-H), 6.98 (1H, dd, J=8.4, 2.4 Hz, Ar-H), 6.94 (1H, d, J= 2.4 $\mathrm{Hz}, \mathrm{Ar}-\mathrm{H}), 6.49(1 \mathrm{H}, \mathrm{d}, J=8.4 \mathrm{~Hz}, \mathrm{Ar}-\mathrm{H}), 5.02\left(1 \mathrm{H}, \mathrm{s}, \mathrm{C}=\mathrm{CH}_{2}\right), 4.44$ $\left(1 \mathrm{H}, \mathrm{s}, \mathrm{C}=\mathrm{CH}_{2}\right), 4.38(1 \mathrm{H}, \mathrm{dd}, J=9.6,3.3 \mathrm{~Hz}, 2-\mathrm{CH}), 4.09(1 \mathrm{H}, \mathrm{s}, \mathrm{NH})$, $3.36(1 \mathrm{H}, \mathrm{dd}, J=5.4,3.6 \mathrm{~Hz}, 4-\mathrm{CH}), 2.10\left(1 \mathrm{H}, \mathrm{ddd}, J=13.2,3.6,3.3 \mathrm{~Hz}, 3-\mathrm{CH}_{2}\right), 1.96(1 \mathrm{H}$, ddd, $\left.J=13.2,9.6,5.4 \mathrm{~Hz}, 3-\mathrm{CH}_{2}\right), 1.81\left(3 \mathrm{H}, \mathrm{s}, \mathrm{CH}_{3}\right) ;{ }^{13} \mathrm{C} \mathrm{NMR}\left(75 \mathrm{MHz}, \mathrm{CDCl}_{3}\right) \delta 148.2$, 144.1, 143.2, 129.7, 128.6, 127.6, 127.2, 126.6, 123.5, 121.4, 115.1, 114.9, 52.4, 43.2, 33.8, 21.0; IR (film) 3399, 3024, 2956, 2922, 2853, 1599, 1490, 1303, 1252, 1086, 896, 807, 756 $\mathrm{cm}^{-1}$; HRMS-EI (m/z): [M+] calcd for $\mathrm{C}_{18} \mathrm{H}_{18} \mathrm{~N} 283.1128$, Found: 283.1129 .

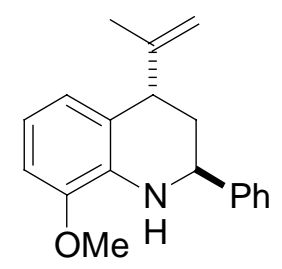

trans-4-Isopropenyl-8-methoxy-2-phenyl-1,2,3,4-tetrahydroquinoline (2g): colorless viscous oil; ${ }^{1} \mathrm{H}$ NMR $\left(300 \mathrm{MHz}, \mathrm{CDCl}_{3}\right) \delta 7.41-7.23(5 \mathrm{H}, \mathrm{m}$, Ar-H), 6.68-6.58 (3H, m, Ar-H), $4.99\left(1 \mathrm{H}, \mathrm{s}, \mathrm{C}=\mathrm{CH}_{2}\right), 4.55(1 \mathrm{H}, \mathrm{s}, \mathrm{NH})$, $4.43\left(1 \mathrm{H}, \mathrm{s}, \mathrm{C}=\mathrm{CH}_{2}\right), 4.39(1 \mathrm{H}, \mathrm{dd}, J=9.6,3.3 \mathrm{~Hz}, 2-\mathrm{CH}), 3.83(3 \mathrm{H}, \mathrm{s}$, $\left.\mathrm{OCH}_{3}\right), 3.43(1 \mathrm{H}, \mathrm{dd}, J=5.7,3.6 \mathrm{~Hz}, 4-\mathrm{CH}), 2.11(1 \mathrm{H}, \mathrm{ddd}, J=13.2,3.6$, $\left.3.3 \mathrm{~Hz}, 3-\mathrm{CH}_{2}\right), 2.01\left(1 \mathrm{H}, \mathrm{ddd}, J=13.2,9.6,5.7 \mathrm{~Hz}, 3-\mathrm{CH}_{2}\right), 1.82\left(3 \mathrm{H}, \mathrm{s}, \mathrm{CH}_{3}\right) ;{ }^{13} \mathrm{C} \mathrm{NMR}(75$ $\left.\mathrm{MHz} \mathrm{CDCl}_{3}\right) \delta 149.1,145.9,144.7,134.6,128.5,127.3,126.7,122.3,121.8,115.6,114.5$, 107.5, 55.3, 51.9, 43.2, 34.3, 21.1; IR (film) 3413, 3058, 3024, 2949, 2853, 1585, 1494, 1450, 1337, 1246, 1100, 1045, 896, $729 \mathrm{~cm}^{-1}$; HRMS-EI (m/z): [M+] calcd for $\mathrm{C}_{19} \mathrm{H}_{21} \mathrm{~N} 279.1623$, Found: 279.1628 .

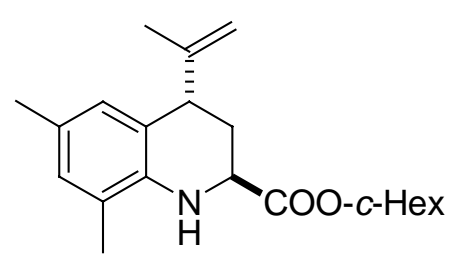

trans-6,8-Dimethyl-4-isopropenyl-1,2,3,4-tetrahydroquinoli ne-2-carboxylic acid cyclohexyl ester (2h): pale yellow viscous oil; ${ }^{1} \mathrm{H}$ NMR (300 MHz, $\left.\mathrm{CDCl}_{3}\right) \delta 6.77(1 \mathrm{H}, \mathrm{s}, \mathrm{Ar}-\mathrm{H})$, $6.64(1 \mathrm{H}, \mathrm{s}, \mathrm{Ar}-\mathrm{H}), 4.99\left(1 \mathrm{H}, \mathrm{s}, \mathrm{C}=\mathrm{CH}_{2}\right), 4.86(1 \mathrm{H}, \mathrm{tt}, J=8.7,3.9$ $\mathrm{Hz}, \mathrm{OCH}), 4.45\left(1 \mathrm{H}, \mathrm{s}, \mathrm{C}=\mathrm{CH}_{2}\right), 4.13(1 \mathrm{H}, \mathrm{s}, \mathrm{NH}), 3.99(1 \mathrm{H}, \mathrm{dd}$, $J=9.9,3.3 \mathrm{~Hz}, 2-\mathrm{CH}), 3.43(1 \mathrm{H}, \mathrm{dd}, J=5.4,3.6 \mathrm{~Hz}, 4-\mathrm{CH}), 2.28(1 \mathrm{H}, \mathrm{ddd}, J=13.2,3.6,3.3$ 
$\left.\mathrm{Hz}, 3-\mathrm{CH}_{2}\right), 2.19\left(3 \mathrm{H}, \mathrm{s}, \mathrm{Ar}-\mathrm{CH}_{3}\right), 2.15\left(3 \mathrm{H}, \mathrm{s}, \mathrm{Ar}-\mathrm{CH}_{3}\right), 1.91(1 \mathrm{H}, \mathrm{ddd}, J=13.2,9.9,5.4 \mathrm{~Hz}$, $\left.3-\mathrm{CH}_{2}\right), 1.87-1.78(2 \mathrm{H}, \mathrm{m}), 1.82\left(3 \mathrm{H}, \mathrm{s}, \mathrm{CH}_{3}\right), 1.76-1.66(2 \mathrm{H}, \mathrm{m}), 1.57-1.24(6 \mathrm{H}, \mathrm{m}) ;{ }^{13} \mathrm{C}$ NMR $\left(75 \mathrm{MHz}, \mathrm{CDCl}_{3}\right) \delta 173.3,147.9,138.8,129.3,127.9,125.9,121.7,121.5,114.9,73.4$, 51.4, 43.4, 31.4, 28.3, 25.3, 23.5, 20.7, 20.4, 17.2; IR (film) 3406, 3072, 2928, 2853, 1732, 1490, 1446, 1215, 1035, 1011, 896, 858, $743 \mathrm{~cm}^{-1}$; HRMS-EI (m/z): [M+ calcd for $\mathrm{C}_{21} \mathrm{H}_{29} \mathrm{~N}$ 327.2198, Found: 327.2203.

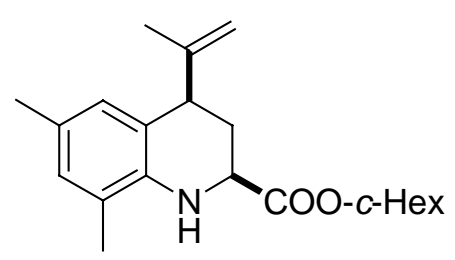

cis-6,8-Dimethyl-4-isopropenyl-1,2,3,4-tetrahydroquinoline2-carboxylic acid cyclohexyl ester (cis-2h): This compound was assigned by only ${ }^{1} \mathrm{H}$ NMR and HRMS because little amount was obtained as a side product; pale yellow viscous oil; ${ }^{1} \mathrm{H}$ NMR $\left(300 \mathrm{MHz}, \mathrm{CDCl}_{3}\right) \delta 6.76(1 \mathrm{H}, \mathrm{s}, \mathrm{Ar}-\mathrm{H}), 6.69(1 \mathrm{H}, \mathrm{s}, \mathrm{Ar}-\mathrm{H})$, 4.99-4.96 (2H, m, NH and $\left.\mathrm{C}=\mathrm{CH}_{2}\right), 4.88(1 \mathrm{H}, \mathrm{tt}, J=8.7,3.6 \mathrm{~Hz}, \mathrm{OCH}), 4.10\left(1 \mathrm{H}, \mathrm{s}, \mathrm{C}=\mathrm{CH}_{2}\right)$, 4.10-4.03 (1H, m, 2-CH), 3.73 (1H, dd, $J=11.7,5.4 \mathrm{~Hz}, 4-\mathrm{CH}), 2.31-2.24(1 \mathrm{H}, \mathrm{m}), 2.19(3 \mathrm{H}$, $\left.\mathrm{s}, \mathrm{Ar}-\mathrm{CH}_{3}\right), 2.14\left(3 \mathrm{H}, \mathrm{s}, \mathrm{Ar}-\mathrm{CH}_{3}\right), 1.93\left(1 \mathrm{H}, \mathrm{ddd}, J=12.3,11.7,11.7 \mathrm{~Hz}, 3-\mathrm{CH}_{2}\right), 1.91-1.84$ $(2 \mathrm{H}, \mathrm{m}), 1.78-1.69(2 \mathrm{H}, \mathrm{m}), 1.59\left(3 \mathrm{H}, \mathrm{s}, \mathrm{CH}_{3}\right), 1.59-1.24(6 \mathrm{H}, \mathrm{m}) ; \mathrm{HRMS}-\mathrm{EI}(\mathrm{m} / \mathrm{z}):\left[\mathrm{M}^{+}\right]$ calcd for $\mathrm{C}_{21} \mathrm{H}_{29} \mathrm{~N}$ 327.2198, Found: 327.2201.

Determination of configuration of 2,4-disubstituted-1,2,3,4-tetrahydro-quinoline 2: The configuration was determined by preparation of trans- and cis-4-isopropyl-2-phenyl-1,2,3,4-tetrahydroquinoline 2i respectively (Scheme 2 and 3).

(1) trans-4-isopropyl-2-phenyl-1,2,3,4-tetrahydroquinoline: To a solution of trans-4-isopropenyl-2-phenyl-1,2,3,4-tetrahydroquinoline 2a (49.3 mg, $0.198 \mathrm{mmol}$, trans only) in $\mathrm{MeOH}(2.0 \mathrm{~mL})$ was added $\mathrm{Pd}-\mathrm{C}(10 \%, 10 \mathrm{mg})$ and the mixture was stirred for $48 \mathrm{~h}$ at room temperature under a $\mathrm{H}_{2}$ atmosphere. The resulting mixture was filtered through a pad of Celite and the filtrate was concentrated. Tne residue was purified by chromatography on silica gel (hexane/ $\mathrm{Et}_{2} \mathrm{O}=40: 1$ to $15: 1$ as eluent) to give trans-4-isopropyl-2-phenyl-1,2,3,4-tetrahydroquinoline as a pale yellow viscous oil (45.5 $\mathrm{mg}, 0.181 \mathrm{mmol}, 91 \%$ yield, trans/cis $=8: 1$ determined by ${ }^{1} \mathrm{H}$ NMR analysis $) .{ }^{1} \mathrm{H} \mathrm{NMR}(300$ $\left.\mathrm{MHz}, \mathrm{CDCl}_{3}\right) \delta$ 7.46-7.23 (5H, m, Ar-H), 7.05-6.99 (2H, m, Ar-H), $6.62(1 \mathrm{H}, \mathrm{td}, J=7.5,1.2$ $\mathrm{Hz}, \mathrm{Ar}-\mathrm{H}), 6.53(1 \mathrm{H}, \mathrm{d}, J=8.4 \mathrm{~Hz}, \mathrm{Ar}-\mathrm{H}), 4.50(1 \mathrm{H}, \mathrm{dd}, J=11.2,3.6 \mathrm{~Hz}, 2-\mathrm{CH}), 4.02(1 \mathrm{H}, \mathrm{br}$, $\mathrm{NH}), 2.44-2.38(1 \mathrm{H}, \mathrm{m}, 4-\mathrm{CH}), 2.17\left(1 \mathrm{H}, \mathrm{ddd}, J=13.5,3.9,3.6 \mathrm{~Hz}, 3-\mathrm{CH}_{2}\right), 2.05-1.92(1 \mathrm{H}$, m, $\left.\mathrm{C} \underline{\mathrm{H}}\left(\mathrm{CH}_{3}\right)_{2}\right), 1.82\left(1 \mathrm{H}, \mathrm{ddd}, J=13.5,11.1,4.5 \mathrm{~Hz}, 3-\mathrm{CH}_{2}\right), 1.02(3 \mathrm{H}, \mathrm{d}, J=6.6 \mathrm{~Hz}$, $\left.\mathrm{CH}\left(\mathrm{CH}_{3}\right)_{2}\right), 0.98\left(3 \mathrm{H}, \mathrm{d}, J=6.6 \mathrm{~Hz}, \mathrm{CH}\left(\mathrm{CH}_{3}\right)_{2}\right) ;{ }^{13} \mathrm{C} \mathrm{NMR}\left(75 \mathrm{MHz}, \mathrm{CDCl}_{3}\right) \delta 145.4,143.9$, 130.1, 128.6, 127.4, 127.0, 126.5, 123.6, 116.0, 113.6, 53.1, 42.8, 33.1, 31.4, 21.6, 19.7; IR 
(film) 3399, 3051, 3017, 2956, 2860, 1606, 1483, 1310, 1256, 1117, 1021, 913, $743 \mathrm{~cm}^{-1}$; HRMS-EI (m/z): $\left[\mathrm{M}^{+}\right]$calcd for $\mathrm{C}_{18} \mathrm{H}_{21} \mathrm{~N} 251.1674$, Found: 251.1671 .

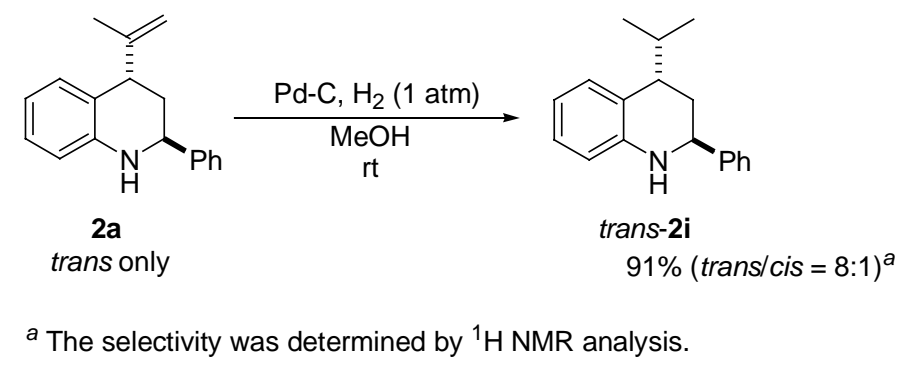

Scheme 2. Reduction of Isopropenyl by Hydrogenation.

(2) cis-4-isopropyl-2-phenyl-1,2,3,4-tetrahydroquinoline: (i) To a mixture of 2-phenyl-4-quinolinecarboxylic acid (1.25 g, $5.00 \mathrm{mmol}), \mathrm{MeOH}(0.61 \mathrm{~mL}, 15 \mathrm{mmol})$ and DMAP (31 mg, $0.25 \mathrm{mmol})$ in $\mathrm{CH}_{2} \mathrm{Cl}_{2}(20 \mathrm{~mL})$ was added a solution of DCC (1.03 $\mathrm{g}, 5.0$ mmol) in $\mathrm{CH}_{2} \mathrm{Cl}_{2}(5 \mathrm{~mL})$ at $0{ }^{\circ} \mathrm{C}$. The mixture was stirred for $1 \mathrm{~h}$ at $0{ }^{\circ} \mathrm{C}$ and for $12 \mathrm{~h}$ at room temperature. The resulting mixture was filtered and the filtrate was concentrated. The residue was purified by column chromatography on silica gel (hexane/ $\mathrm{Et}_{2} \mathrm{O}=10: 1$ to $5: 1$ as eluent) to obtain the corresponding methyl ester as colorless crystals (1.30 g, $4.94 \mathrm{mmol}$, $99 \%$ yield). (ii) To a solution of methyl ester (263 mg, $1.00 \mathrm{mmol})$ in $\mathrm{Et}_{2} \mathrm{O}(2.0 \mathrm{~mL})$ was added a $1 \mathrm{M} \mathrm{MeMgI}$ solution in $\mathrm{Et}_{2} \mathrm{O}(5.0 \mathrm{~mL}, 5.0 \mathrm{mmol})$ at $0^{\circ} \mathrm{C}$. After stirring for $36 \mathrm{~h}$ at room temperature, the resulting mixture was quenched with saturated $\mathrm{NH}_{4} \mathrm{Cl}$ at $0{ }^{\circ} \mathrm{C}$ and extracted with $\mathrm{Et}_{2} \mathrm{O}$. The combined extracts were washed with saturated $\mathrm{NaHCO}_{3}$ and brine, dried over $\mathrm{Na}_{2} \mathrm{SO}_{4}$ and concentrated. The crude tert-alcohol was obtained as colorless crystals (251 mg, $0.953 \mathrm{mmol}, 95 \%$ yield) and it was used without purification in next step. The crude tert-alcohol $(79 \mathrm{mg}, 0.30 \mathrm{mmol})$ was dissolved in pyridine $(1.0 \mathrm{~mL})$ and treated with $\mathrm{SOCl}_{2}(2.0 \mathrm{~mL})$ at $0{ }^{\circ} \mathrm{C}$ and the mixture was stirred for $2 \mathrm{~h}$ at room temperature. The volatiles were removed in vacuo (high-vacuum with heating) and saturated $\mathrm{NaHCO}_{3}$ was added to the residue. The mixture was extracted with $\mathrm{Et}_{2} \mathrm{O}$ and the combined extracts were washed with brine and dried over $\mathrm{Na}_{2} \mathrm{SO}_{4}$. Removal of the solvents and purification of the residue by column chromatography on silica gel (hexane/ $\mathrm{Et}_{2} \mathrm{O}=20: 1$ to $10: 1$ as eluent) gave 4-isopropenyl-2-phenylquinoline as a colorless viscous oil $(43.9 \mathrm{mg}, 0.179 \mathrm{mmol}, 60 \%$ yield). (iii) A mixture of 4-isopropenyl-2-phenylquinoline (112 mg, $0.446 \mathrm{mmol}$ ) and $\mathrm{PtO}_{2}$ (5.0 mg, $0.022 \mathrm{mmol})$ in $\mathrm{MeOH}(4.5 \mathrm{~mL})$ was stirred for $16 \mathrm{~h}$ under a $\mathrm{H}_{2}$ atmosphere. After removal of the catalyst by Celite filtration, the volatiles were removed by evaporation. The 
residue was purified by column chromatography on silica gel (hexane/ $\mathrm{Et}_{2} \mathrm{O}=40: 1$ to 20:1) to afford cis-4-isopropyl-2-phenyl-1,2,3,4-tetrahydroquinoline as a colorless viscous oil (66.1 $\mathrm{mg}, 0.263 \mathrm{mmol}, 59 \%$ yield, trans/cis $=1:>20$ determined by ${ }^{1} \mathrm{H}$ NMR analysis). Similar selectivities have been previously observed in $\mathrm{PtO}_{2}$ catalyzed hydrogenations of substituted quinolines. $^{6}$
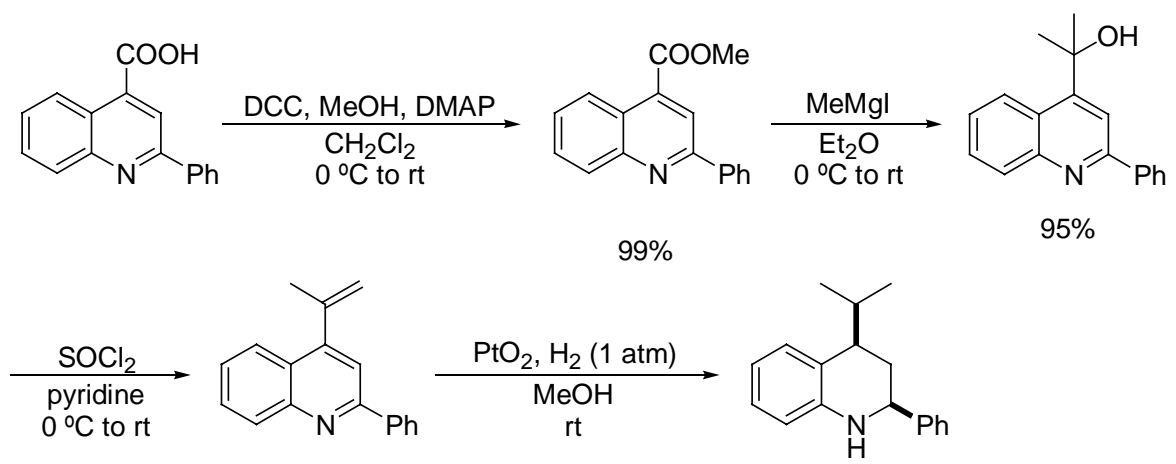

$60 \%$

a The selectivity was determined by ${ }^{1} \mathrm{H}$ NMR analysis.

$59 \%(\text { trans } / \text { cis }=1:>20)^{a}$

Scheme 3. Determination of Configuration of 2,4-Disubstituted-1,2,3,4-Tetrahydroquinoline.

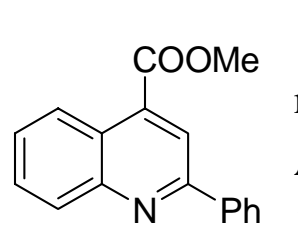

2-Phenyl-4-quinolinecarboxylic acid methyl ester ${ }^{7}$ : colorless crystal, $\mathrm{mp}=60-61^{\circ} \mathrm{C} ;{ }^{1} \mathrm{H} \mathrm{NMR}\left(300 \mathrm{MHz}, \mathrm{CDCl}_{3}\right) \delta 8.74(1 \mathrm{H}, \mathrm{dd}, J=8.4,0.6 \mathrm{~Hz}$, Ar-H), 8.40 (1H, s, Ar-H), 8.24-8.19 (3H, m, Ar-H), $7.76(1 \mathrm{H}, \mathrm{td}, J=7.8$, $1.5 \mathrm{~Hz}, \mathrm{Ar}-\mathrm{H}), 7.62(1 \mathrm{H}, \mathrm{td}, J=7.8,1.5 \mathrm{~Hz}, \mathrm{Ar}-\mathrm{H}), 7.57-7.45(3 \mathrm{H}, \mathrm{m}$, $\mathrm{Ar}-\mathrm{H}), 4.06\left(3 \mathrm{H}, \mathrm{s}, \mathrm{COOCH}_{3}\right) ;{ }^{13} \mathrm{C} \mathrm{NMR}\left(75 \mathrm{MHz}, \mathrm{CDCl}_{3}\right) \delta 166.8,156.7,149.2,138.8$, 135.5, 130.3, 129.9, 129.7, 128.9, 127.8, 127.4, 125.4, 124.0, 120.3, 52.7 (d, J = 3 Hz); IR (film) 3058, 2949, 1725, 1589, 1548, 1433, 1344, 1249, 1198, 1147, 1028, 1015, 947, 899, $767 \mathrm{~cm}^{-1}$; HRMS-EI (m/z): $\left[\mathrm{M}^{+}\right]$calcd for $\mathrm{C}_{17} \mathrm{H}_{13} \mathrm{NO}_{2} 263.0946$, Found: 263.0954 .

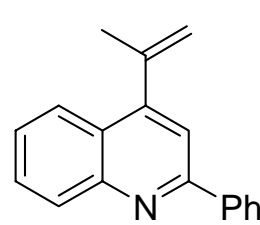

4-Isopropenyl-2-phenylquinoline: colorless viscous oil; ${ }^{1} \mathrm{H}$ NMR (300 $\left.\mathrm{MHz}, \mathrm{CDCl}_{3}\right) \delta$ 8.20-8.14 (3H, m, Ar-H), $8.04(1 \mathrm{H}, \mathrm{dd}, J=8.4,0.9 \mathrm{~Hz}$, Ar-H), $7.71(1 \mathrm{H}, \mathrm{td}, J=7.8,1.5 \mathrm{~Hz}, \mathrm{Ar}-\mathrm{H}), 7.69(1 \mathrm{H}, \mathrm{s}, \mathrm{Ar}-\mathrm{H}), 7.55-7.43$ $\left.0.9 \mathrm{~Hz}, \mathrm{C}=\mathrm{CH}_{2}\right), 2.26\left(3 \mathrm{H}, \mathrm{dd}, J=1.5,0.9 \mathrm{~Hz}, \mathrm{CH}_{3}\right) ;{ }^{13} \mathrm{C} \mathrm{NMR}\left(75 \mathrm{MHz}, \mathrm{CDCl}_{3}\right) \delta 157.0$, $151.3,148.7,142.5,139.8,130.2$, 129.4, 129.2, 128.8, 127.5, 126.1, 125.3, 125.1, 117.3, 
117.2, 24.6; IR (film) 3058, 2962, 1585, 1545, 1494, 1443, 1351, 1232, 1076, 1028, 906, 882, $770 \mathrm{~cm}^{-1}$; HRMS-EI (m/z): [ $\left.\mathrm{M}^{+}\right]$calcd for $\mathrm{C}_{18} \mathrm{H}_{15} \mathrm{~N}$ 245.1205, Found: 245.1199 .

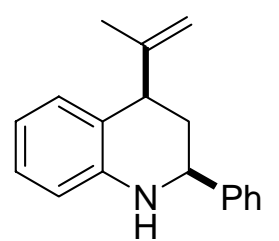

\section{cis-4-Isopropyl-2-phenyl-1,2,3,4-tetrahydroquinoline:} colorless viscous oil; ${ }^{1} \mathrm{H}$ NMR $\left(300 \mathrm{MHz}, \mathrm{CDCl}_{3}\right) \delta$ 7.46-7.42 $(2 \mathrm{H}, \mathrm{m}$, Ar-H), $7.37(2 \mathrm{H}, \mathrm{tt}, J=7.5,1.2 \mathrm{~Hz}, \mathrm{Ar}-\mathrm{H}), 7.30(1 \mathrm{H}, \mathrm{tt}, J=7.5,1.2 \mathrm{~Hz}$, Ar-H), $7.18(1 \mathrm{H}, \mathrm{d}, J=7.2 \mathrm{~Hz}, \mathrm{Ar}-\mathrm{H}), 6.99(1 \mathrm{H}, \mathrm{tt}, J=7.5,1.2 \mathrm{~Hz}, \mathrm{Ar}-\mathrm{H})$, $6.72(1 \mathrm{H}, \mathrm{td}, J=7.5,1.2 \mathrm{~Hz}, \mathrm{Ar}-\mathrm{H}), 6.54(1 \mathrm{H}, \mathrm{dd}, J=7.5,1.2 \mathrm{~Hz}, \mathrm{Ar}-\mathrm{H}), 4.37(1 \mathrm{H}, \mathrm{dd}, J=$ 11.4, $2.7 \mathrm{~Hz}, 2-\mathrm{CH}), 3.89(1 \mathrm{H}, \mathrm{br}, \mathrm{NH}), 3.12-3.05(1 \mathrm{H}, \mathrm{m}, 4-\mathrm{CH}), 2.51(1 \mathrm{H}, \mathrm{qqd}, J=6.9,6.9$, $\left.3.9 \mathrm{~Hz}, \mathrm{CH}\left(\mathrm{CH}_{3}\right)_{2}\right), 2.01\left(1 \mathrm{H}, \mathrm{ddd}, J=12.9,5.7,2.7 \mathrm{~Hz}, 3-\mathrm{CH}_{2}\right), 1.76(1 \mathrm{H}, \mathrm{dd}, J=12.9,11.4$ $\left.\mathrm{Hz}, 3-\mathrm{CH}_{2}\right), 1.05\left(3 \mathrm{H}, \mathrm{d}, J=6.9 \mathrm{~Hz}, \mathrm{CH}\left(\mathrm{CH}_{3}\right)_{2}\right), 0.73\left(3 \mathrm{H}, \mathrm{d}, J=6.9 \mathrm{~Hz}, \mathrm{CH}\left(\mathrm{CH}_{3}\right)_{2}\right) ;{ }^{13} \mathrm{C}$ NMR $\left(75 \mathrm{MHz}, \mathrm{CDCl}_{3}\right) \delta 146.2,144.8,128.6,127.6,127.0,126.6,126.5,124.6,117.9$, 114.7, 57.2, 41.6, 32.1, 29.3, 20.9, 15.5; IR (film) 3365, 3024, 2949, 2867, 1602, 1477, 1334, 1310, 1252, 1184, 1113, 919, 845, $746 \mathrm{~cm}^{-1}$; HRMS-EI $(\mathrm{m} / \mathrm{z})$ : $\left[\mathrm{M}^{+}\right]$calcd for $\mathrm{C}_{18} \mathrm{H}_{21} \mathrm{~N}$ 251.1674, Found: 251.1666.

\section{References}

(1) Ukai, T.; Kawazura, H.; Ishii, Y. J. Organomet. Chem. 1994, 65, 253.

(2) Kosynkin, D. V.; Tour, J. M. Org. Lett. 2001, 3, 991.

(3) Kondo, Y.; Kojima, S.; Sakamoto, T. J. Org. Chem. 1997, 62, 6507.

(4) Roesch, K. R.; Larock, R. C. J. Org. Chem. 2001, 66, 412.

(5) Fernández, F.; García, G.; Rodríguez, J. E. Synth. Comm. 1990, 20, 2837.

(6) (a) Nagata, R.; Ae, N.; Tanno, N. Bioorganic \& Medicinal Chem. Lett. 1995, 5, 1527. (b) Carling, R. W.; Leeson, P. D.; Moseley, A. M.; Baker, R.; Foster, A. C.; Grimwood, S.; Kemp, J. A.; Marshall, G. R. J. Med. Chem. 1992, 35, 1942.

(7) Commercially available from Aldrich.

\section{Preparation of iodophenols derivatives}

Iodophenols derivatives (1-iodonaphthol, 2-iodo-5-trifluoromethylphenol and 3-hydroxy-4-iodo-benzoic acid methyl ester) were prepared according to procedures 
described in the literature. ${ }^{1}$ The iodinated compounds were purified by chromatography over silica gel using mixtures of hexanes/ethyl acetate as eluent: (1-iodonaphthol : 74\%, Hexanes:EtOAc, 95:5); (2-iodo-5-trifluoromethylphenol : 75\%, Hexanes:EtOAc, 9:1); (3-hydroxy-4-iodo-benzoic acid methyl ester : 75\%, Hexanes:EtOAc, 9:1). $N$-(2-Iodo-phenyl)-4-methyl-benzenesulfonamide and 2-(2-iodophenyl)-malonic acid diethyl ester were prepared according to procedures described in the literature. ${ }^{2}$

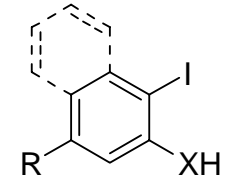

$(\mathrm{X}=\mathrm{O}, \mathrm{NTs})$

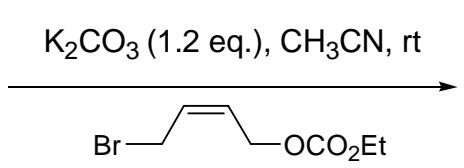

$-\mathrm{OCO}_{2} \mathrm{Et}$

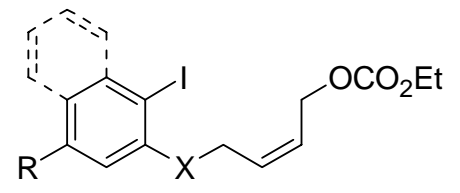

3a-d

To a solution of the aromatic substrate (iodophenol derivative or tosylated aniline derivative) $(1$ eq. $)$ in dry $\mathrm{CH}_{3} \mathrm{CN} \quad(\mathrm{C}=0.06 \quad \mathrm{M})$ were added $\mathrm{K}_{2} \mathrm{CO}_{3} \quad(1.2$ eq. $)$ and (Z) $-\mathrm{BrCH}_{2} \mathrm{CH}=\mathrm{CHCH}_{2} \mathrm{OCO}_{2} \mathrm{Et}$ (1.1 eq.). The reaction mixture was stirred at room temperature for $3-24 \mathrm{~h}$ depending on the starting material (see table below). The reaction was quenched with water and extracted with diethyl ether. The organic layer was dried over $\mathrm{Na}_{2} \mathrm{SO}_{4}$ and concentrated under reduced pressure. The residue was purified by chromatography over silica gel with mixtures of hexanes/EtOAc.

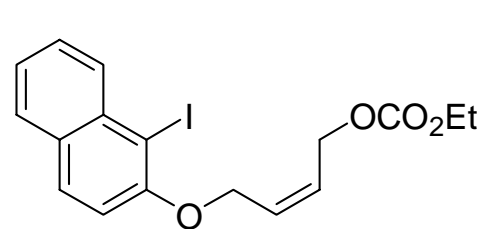

Carbonic

acid

ethyl

ester 4-(1-iodo-naphthalen-2-yloxy)-but-2-enyl ester (3a): $400 \mathrm{MHz}) 8.14(\mathrm{~d}, J=8.4 \mathrm{~Hz}, 1 \mathrm{H}), 7.77(\mathrm{~d}, J=8.8 \mathrm{~Hz}, 1 \mathrm{H}), 7.72(\mathrm{~d}, J=8.2 \mathrm{~Hz}, 1 \mathrm{H}), 7.53(\mathrm{t}$, $J=8.4 \mathrm{~Hz}, 1 \mathrm{H}), 7.38(\mathrm{t}, J=8.1 \mathrm{~Hz}, 1 \mathrm{H}), 7.15(\mathrm{~d}, J=8.8 \mathrm{~Hz}, 1 \mathrm{H}), 6.07-6.01(\mathrm{~m}, 1 \mathrm{H})$, 5.87-5.81 (m, 1H), $4.85(\mathrm{~d}, J=5.8 \mathrm{~Hz}, 2 \mathrm{H}), 4.81(\mathrm{~d}, J=6.6 \mathrm{~Hz}, 2 \mathrm{H}), 4.21(\mathrm{q}, J=7.3 \mathrm{~Hz}, 2 \mathrm{H})$, $1.31(\mathrm{t}, J=7.3 \mathrm{~Hz}, 3 \mathrm{H}) ;{ }^{13} \mathrm{C} \mathrm{NMR}\left(\mathrm{CDCl}_{3}, 100 \mathrm{MHz}\right) 155.47,154.37,135.49,131.22,130.17$, 129.99, 129.61, 128.06, 127.99, 126.90, 124.47, 114.41, 88.95, 66.00, 64.07, 63.33, 14.19;

\footnotetext{
${ }^{1}$ Adimurthy, S., Ramachandraiah, G., Ghosh, P. K., Bedekar, A. V. Tetrahedron Lett., 2003, 44, 5099.

2 A) Zenner, J. M.; Larock, R. C. J. Org. Chem. 1999, 64, 7312. b) Cockerill, G. S., Levett, P. C., Whiting, D. A. J. Chem. Soc. Perkin Trans. 1, 1995, 1103.
} 
IR (film) 2982, 2931, 2906, 1743, 1621, 1592, 1501, 1460, 1370, 1349, 1255, 1149, 1049, 1018, $873 \mathrm{~cm}^{-1}$; HRMS-ESI $(\mathrm{m} / \mathrm{z}):[\mathrm{M}+\mathrm{Na}]^{+}$calcd for $\mathrm{C}_{17} \mathrm{H}_{17} \mathrm{O}_{4} \mathrm{INa}$ 435.0063, found 435.0077 .

$\begin{array}{ll}\mathrm{OCO}_{2} \mathrm{Et} & \begin{array}{l}\text { Carbonic acid } \\ \text { 4-(2-iodo-5-trifluoromethyl-phenoxy)-but-2-enyl ester } \\ (\mathbf{3 b}) \text { : colorless viscous oil, purified by chromatography over }\end{array}\end{array}$ silica gel (81\%, Hexanes:EtOAc, 95:5, Rf =0.3); ${ }^{1} \mathrm{H} \mathrm{NMR}\left(\mathrm{CDCl}_{3}, 400 \mathrm{MHz}\right) 7.89(\mathrm{~d}, J=$ $8.1 \mathrm{~Hz}, 1 \mathrm{H}), 7.01(\mathrm{~s}, 1 \mathrm{H}), 6.97(\mathrm{~d}, J=8.8 \mathrm{~Hz}, 1 \mathrm{H}), 6.00-5.95(\mathrm{~m}, 1 \mathrm{H}), 5.89-5.83(\mathrm{~m}, 1 \mathrm{H})$, $4.79(\mathrm{~m}, 4 \mathrm{H}), 4.21(\mathrm{q}, J=7.3 \mathrm{~Hz}, 2 \mathrm{H}), 1.31(\mathrm{t}, J=7.3 \mathrm{~Hz}, 3 \mathrm{H}) ;{ }^{13} \mathrm{C} \mathrm{NMR}\left(\mathrm{CDCl}_{3}, 100 \mathrm{MHz}\right)$ $157.19,154.86,139.99,131.77(\mathrm{q}, J=32.8 \mathrm{~Hz}), 128.55,127.42,123.59$ (q, $J=272.7 \mathrm{~Hz})$, $119.18(\mathrm{q}, J=3.8 \mathrm{~Hz}), 108.52(\mathrm{q}, J=3.8 \mathrm{~Hz}), 91.03(\mathrm{q}, J=1.5 \mathrm{~Hz}), 65.28,64.08,63.23$, 14.11; IR (film) 2985, 2931, 2869, 1745, 1595, 1580, 1481, 1416, 1377, 1327, 1261, 1170, 1128, 1083, 1017, 874, $791 \mathrm{~cm}^{-1}$; HRMS-ESI (m/z): $[\mathrm{M}+\mathrm{Na}]^{+}$calcd for $\mathrm{C}_{14} \mathrm{H}_{14} \mathrm{O}_{4} \mathrm{~F}_{3} \mathrm{INa}$ 452.9781, found 452.9770 .

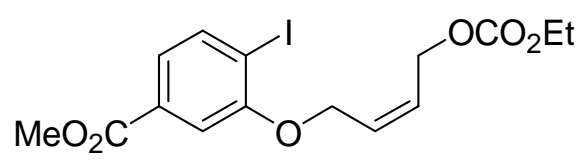

3-(4-Ethoxycarbonyloxy-but-2-enyloxy)-4-iodo-benz oic acid methyl ester (3c): white solid, purified by chromatography over silica gel $(91 \%$, Hexanes:EtOAc, 9:1, Rf=0.2); M.p. 57-59 ${ }^{\circ} \mathrm{C} ;{ }^{1} \mathrm{H} \mathrm{NMR}\left(\mathrm{CDCl}_{3}, 400 \mathrm{MHz}\right) 7.79(\mathrm{~d}, J=8.1 \mathrm{~Hz}, 1 \mathrm{H}), 7.35$ (d, $J=1.5 \mathrm{~Hz}, 1 \mathrm{H}), 7.31(\mathrm{dd}, J=8.1 \mathrm{~Hz}, J=1.5 \mathrm{~Hz}, 1 \mathrm{H}), 5.97-5.91(\mathrm{~m}, 1 \mathrm{H}), 5.83-5.76(\mathrm{~m}, 1 \mathrm{H})$, $4.76(\mathrm{~d}, J=6.6 \mathrm{~Hz}, 2 \mathrm{H}), 4.71(\mathrm{~d}, J=6.0 \mathrm{~Hz}, 2 \mathrm{H}), 4.15(\mathrm{q}, J=7.3 \mathrm{~Hz}, 2 \mathrm{H}), 3.85(\mathrm{~s}, 3 \mathrm{H}), 1.25$ $(\mathrm{t}, J=7.3 \mathrm{~Hz}, 3 \mathrm{H}) ;{ }^{13} \mathrm{C} \mathrm{NMR}\left(\mathrm{CDCl}_{3}, 100 \mathrm{MHz}\right) 166.14,156.87,154.78,139.47,131.36$, 128.57, 127.28, 123.47, 112.23, 93.03, 65.05, 63.98, 63.32, 52.17, 14.11; IR (film) 2984, 2952, 2906, 1745, 1723, 1585, 1571, 1475, 1436, 1406, 1377, 1291, 1257, 1109, 1017, 874, 791, $759 \mathrm{~cm}^{-1}$; HRMS-ESI $(\mathrm{m} / \mathrm{z})$ : $[\mathrm{M}+\mathrm{Na}]^{+}$calcd for $\mathrm{C}_{15} \mathrm{H}_{17} \mathrm{O}_{6} \mathrm{INa}$ 442.9962, found 442.9963.

Carbonic acid ethyl ester 4-[(2-iodo-phenyl)-(toluene-4-sulfonyl)-amino]-but-2-enyl $\mathrm{OCO}_{2} \mathrm{Et}$
ester (3d): white solid, purified by chromatography over silica
gel $\left(95 \%\right.$, Hexanes:EtOAc, 85:15, Rf =0.2); M.p. $76-78{ }^{\circ} \mathrm{C} ;{ }^{1} \mathrm{H}$
$\mathrm{NMR}\left(400 \mathrm{MHz}, \mathrm{CDCl}_{3}\right) 7.89(\mathrm{~d}, J=8.1 \mathrm{~Hz}, 1 \mathrm{H}), 7.67(\mathrm{~d}, J=8.4$ $\mathrm{Hz}, 2 \mathrm{H}), 7.31-7.25(\mathrm{~m}, 3 \mathrm{H}), 7.02(\mathrm{t}, J=7.7 \mathrm{~Hz}, 1 \mathrm{H}), 6.95(\mathrm{~d}, J=8.1 \mathrm{~Hz}, 1 \mathrm{H}), 5.80-5.74(\mathrm{~m}$, 
1H), 5.65-5.59 (m, 1H), 4.42-4.18 (m, 4H), $4.14(\mathrm{q}, J=7.3 \mathrm{~Hz}, 2 \mathrm{H}), 2.44(\mathrm{~s}, 3 \mathrm{H}), 1.27(\mathrm{t}, J=$ $7.3 \mathrm{~Hz}, 3 \mathrm{H}) ;{ }^{13} \mathrm{C}$ NMR (100 MHz, $\left.\mathrm{CDCl}_{3}\right)$ 154.71, 143.83, 140.98, 140.36, 136.10, 130.62, 129.97, 129.54, 128.71, 128.45, 128.08, 127.45, 103.00, 64.00, 62.41, 48.05, 21.53, 14.17; IR (film) 3053, 3022, 2983, 2924, 2869, 1745, 1597, 1466, 1440, 1375, 1352, 1258, 1164, 1092, 1018, 873, 715, $658 \mathrm{~cm}^{-1}$; HRMS-ESI $(\mathrm{m} / \mathrm{z}):[\mathrm{M}+\mathrm{Na}]^{+}$calcd for $\mathrm{C}_{20} \mathrm{H}_{22} \mathrm{NO}_{5} \mathrm{SINa}$ 538.0155, found 538.0137.
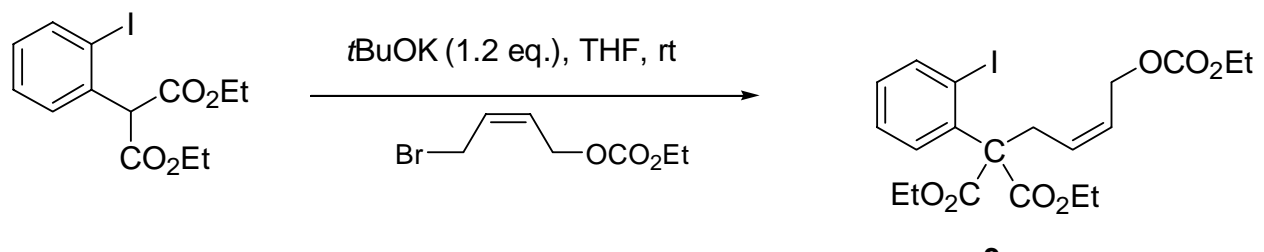

$3 e$

To a solution of 2-(2-iodophenyl)-malonic acid diethyl ester (1 eq.) in $\operatorname{dry} \operatorname{THF}(\mathrm{C}=0.05 \mathrm{M})$ were added potassium tert-butoxide (1.2 eq.) and $(Z)-\mathrm{BrCH}_{2} \mathrm{CH}=\mathrm{CHCH}_{2} \mathrm{OCO}_{2} \mathrm{Et}$ (1.1 eq.). The reaction mixture was stirred at room temperature for $48 \mathrm{~h}$. The reaction was quenched with water and extracted with diethyl ether. The organic layer was dried over $\mathrm{Na}_{2} \mathrm{SO}_{4}$ and concentrated under reduced pressure. The residue was purified by chromatography over silica gel (Hexanes:EtOAc, 9:1) to give $\mathbf{3 e}$ as a colorless viscous oil.

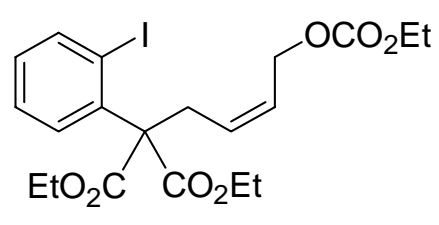

\section{1-(4-Ethoxycarbonyloxy-but-2-enyl)-2-(2-iodo-phenyl)-malo} nic acid diethyl ester (3e): colorless viscous oil, purified by chromatography over silica gel $(87 \%$, Hexanes:EtOAc, 9:1, Rf $=$ $0.2) ;{ }^{1} \mathrm{H} \mathrm{NMR}\left(\mathrm{CDCl}_{3}, 400 \mathrm{MHz}\right) 7.91(\mathrm{dd}, J=8.1 \mathrm{~Hz}, J=1.5 \mathrm{~Hz}$, $1 \mathrm{H}), 7.29(\mathrm{td}, J=8.1 \mathrm{~Hz}, J=1.5 \mathrm{~Hz}, 1 \mathrm{H}), 7.05(\mathrm{dd}, J=8.1 \mathrm{~Hz}, J=1.5 \mathrm{~Hz}, 1 \mathrm{H}), 6.93(\mathrm{td}, J=$ $8.1 \mathrm{~Hz}, J=1.5 \mathrm{~Hz}, 1 \mathrm{H}), 5.57-5.46(\mathrm{~m}, 2 \mathrm{H}), 4.64(\mathrm{~d}, J=5.9 \mathrm{~Hz}, 2 \mathrm{H}), 4.35-4.19(\mathrm{~m}, 4 \mathrm{H}), 4.16$ $(\mathrm{q}, J=7.3 \mathrm{~Hz}, 2 \mathrm{H}), 3.30(\mathrm{~d}, J=5.9 \mathrm{~Hz}, 2 \mathrm{H}), 1.29-1.24(\mathrm{~m}, 9 \mathrm{H}) ;{ }^{13} \mathrm{C} \mathrm{NMR}\left(\mathrm{CDCl}_{3}, 100 \mathrm{MHz}\right)$ $169.31,154.93,142.16,139.45,130.10,129.63,128.81,127.80,125.52,97.49,66.15,63.76$, 63.59, 62.16, 32.55, 14.16, 13.71; IR (film) 2982, 2937, 2900, 1744, 1466, 1440, 1388, 1374, 1255, 1201, 1095, 1060, 1013, 872, 792, 743; HRMS-ESI $(\mathrm{m} / \mathrm{z}):[\mathrm{M}+\mathrm{Na}]^{+}$calcd for $\mathrm{C}_{20} \mathrm{H}_{25} \mathrm{O}_{7} \mathrm{INa} 527.0537$, found 527.0519.

\begin{tabular}{|l|l|l|l|l|}
\hline substrates & $\mathbf{R}$ & $\mathbf{X}$ & Reaction time (h) & Yield (\%) \\
\hline
\end{tabular}




\begin{tabular}{|c|c|c|c|c|}
\hline 3a & $\mathrm{H}$, naphthalene & $\mathrm{O}$ & 3 & 76 \\
\hline 3b & $\mathrm{CF}_{3}$ & $\mathrm{O}$ & 24 & 81 \\
\hline 3c & $\mathrm{CO}_{2} \mathrm{Me}$ & $\mathrm{O}$ & 3 & 91 \\
\hline 3d & $\mathrm{H}$ & $\mathrm{NTs}$ & 24 & 95 \\
\hline 3e & $\mathrm{H}$ & $\mathrm{C}\left(\mathrm{CO}_{2} \mathrm{Et}\right)_{2}$ & 48 & 87 \\
\hline
\end{tabular}
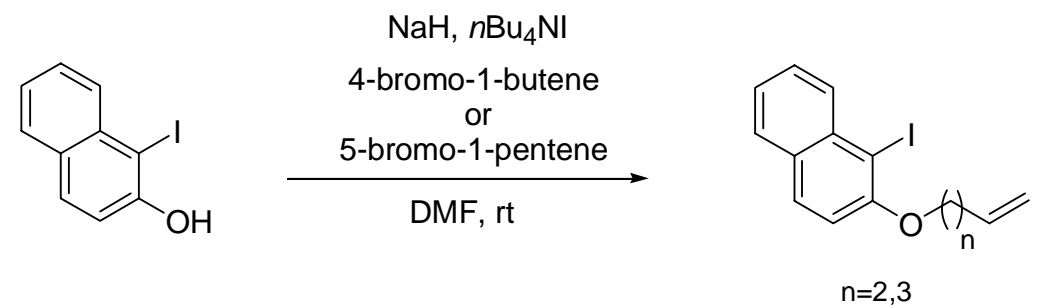

To a suspension of $\mathrm{NaH}$ (1.3 eq.) in $\operatorname{DMF}(\mathrm{C}=0.15 \mathrm{M})$ was added 1-iodonaphthol (1 eq.) at room temperature. After 10 minutes were added $n \mathrm{Bu}_{4} \mathrm{NI}(10 \mathrm{~mol} \%)$ and 4-bromo-1-butene or 5-bromo-1-pentene (1.2 eq.). After stirring at room temperature for $2 \mathrm{~h}$, the mixture was poured into water $(100 \mathrm{~mL})$. The aqueous layer was extracted with $\mathrm{Et}_{2} \mathrm{O}$. The organic layer was washed with water and brine, and dried over $\mathrm{Na}_{2} \mathrm{SO}_{4}$. After concentration under reduced pressure, the residue was purified by chromatography over silica gel (Hexanes:EtOAc, 98:2) to give the alkylated products with 4-bromo-1-butene or 5-bromo-1-pentene as colorless viscous oils in $54 \%$ and $91 \%$ yields respectively.

2-But-3-enyloxy-1-iodo-naphtalene: colorless viscous oil,
purified by chromatography over silica gel $(54 \%$, Hexanes:EtOAc,
$95: 5, \mathrm{Rf}=0.46)$; ${ }^{1} \mathrm{H} \mathrm{NMR}\left(\mathrm{CDCl}_{3}, 400 \mathrm{MHz}\right) 8.17(\mathrm{~d}, J=8.4 \mathrm{~Hz}$, $1 \mathrm{H}), 7.78(\mathrm{~d}, J=8.8 \mathrm{~Hz}, 1 \mathrm{H}), 7.73(\mathrm{~d}, J=8.0 \mathrm{~Hz}, 1 \mathrm{H}), 7.54(\mathrm{ddd}, J=8.4 \mathrm{~Hz}, J=7.0 \mathrm{~Hz}, J=$ $1.5 \mathrm{~Hz}, 1 \mathrm{H}), 7.39$ (ddd, $J=8.0 \mathrm{~Hz}, J=7.0 \mathrm{~Hz}, J=1.1 \mathrm{~Hz}, 1 \mathrm{H}), 7.15(\mathrm{~d}, J=8.8 \mathrm{~Hz}, 1 \mathrm{H})$, 6.10-5.99 (tdd, $J=17.2 \mathrm{~Hz}, J=10.2 \mathrm{~Hz}, J=7.0 \mathrm{~Hz}, 1 \mathrm{H}), 5.28-5.23(\operatorname{tdd}, J=17.2 \mathrm{~Hz}, J=1.8$ $\mathrm{Hz}, J=1.4 \mathrm{~Hz}, 1 \mathrm{H}), 5.19-5.16$ (tdd $J=10.2 \mathrm{~Hz}, J=1.8 \mathrm{~Hz}, J=1.4 \mathrm{~Hz}, 1 \mathrm{H}), 4.20(\mathrm{~m}, 2 \mathrm{H})$, $2.67(\mathrm{~m}, 2 \mathrm{H}) ;{ }^{13} \mathrm{C} \mathrm{NMR}\left(\mathrm{CDCl}_{3}, 100 \mathrm{MHz}\right) 155.99,135.62,134.32,131.24,130.16,129.92$, 128.10, 127.96, 124.32, 117.31, 114.28, 88.73, 69.68, 33.91; IR (film) 3064.6, 2931.1, 2869.5, 1638.7, 1621.5, 1593.0, 1501.2, 1457.9, 1426.6, 1345.4, 1328.4, 1266.5, 1241.5, 1148.5, 1056.6, 1023.8, 917.6, 801.2, 762.9, 745.2 cm-1 ; HRMS-EI (m/z): $\left[\mathrm{M}^{+}\right]$calcd for $\mathrm{C}_{14} \mathrm{H}_{13} \mathrm{OI} 324.001117$, found 324.001091. 


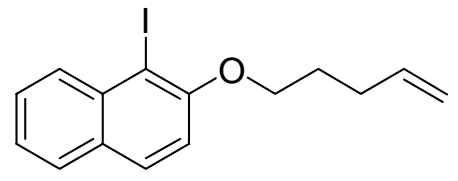

1-Iodo-2-pent-4-enyloxy-naphthalene: colorless viscous oil, purified by chromatography over silica gel (91\%, Hexanes:EtOAc, 95:5, $\mathrm{Rf}=0.5) ;{ }^{1} \mathrm{H} \mathrm{NMR}\left(\mathrm{CDCl}_{3}, 400 \mathrm{MHz}\right)$ $8.13(\mathrm{~d}, J=8.4 \mathrm{~Hz}, 1 \mathrm{H}), 7.75(\mathrm{~d}, J=8.8 \mathrm{~Hz}, 1 \mathrm{H}), 7.70(\mathrm{~d}, J=8.1 \mathrm{~Hz}, 1 \mathrm{H}), 7.51(\mathrm{t}, J=8.4 \mathrm{~Hz}$, $1 \mathrm{H}), 7.35(\mathrm{t}, J=8.1 \mathrm{~Hz}, 1 \mathrm{H}), 7.12(\mathrm{~d}, J=8.8 \mathrm{~Hz}, 1 \mathrm{H}), 5.93-5.83(\mathrm{~m}, 1 \mathrm{H}), 5.10(\mathrm{~d}, J=17.2 \mathrm{~Hz}$, $1 \mathrm{H}), 5.01(\mathrm{~d}, J=10.2 \mathrm{~Hz}, 1 \mathrm{H}), 4.15(\mathrm{t}, J=6.2 \mathrm{~Hz}, 2 \mathrm{H}), 2.39-2.34(\mathrm{~m}, 2 \mathrm{H}), 2.93-1.93(\mathrm{~m}, 2 \mathrm{H})$; ${ }^{13} \mathrm{C}$ NMR $\left(\mathrm{CDCl}_{3}, 100 \mathrm{MHz}\right) 156.07,137.70,135.63,131.19,130.15,129.87,128.10$, 127.95, 124.25, 115.33, 114.20, 88.51, 69.31, 30.19, 28.58; IR (film) 3060.6, 2939.2, 2873.1, 2843.8, 1621.6, 1593.6, 1500.5, 1457.8, 1426.6, 1345.7, 1328.4, 1265.4, 1242.0, 1148.5, 1131.7, 1060.3, 1021.1, 912.5, 802.3, 762.8, 745.2 $\mathrm{cm}^{-1}$; HRMS-EI (m/z): $\left[\mathrm{M}^{+}\right]$calcd for $\mathrm{C}_{15} \mathrm{H}_{15} \mathrm{OI} 338.016767$, found 338.016410.
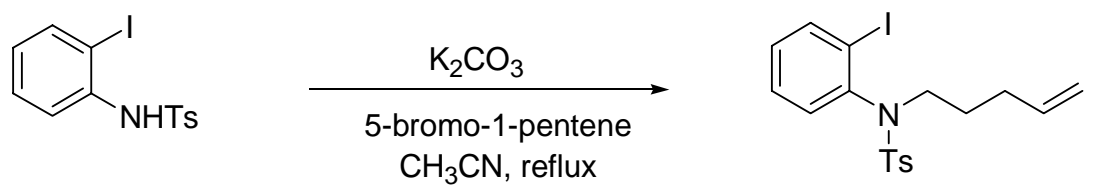

To a solution of tosylated 2-iodoaniline (1 eq.) in $\mathrm{CH}_{3} \mathrm{CN}$ were added $\mathrm{K}_{2} \mathrm{CO}_{3}$ (3 eq.) and 5-bromo-1-pentene (1.2 eq.). After stirring at reflux for $24 \mathrm{~h}$, the mixture was quenched with water. The aqueous layer was extracted with $\mathrm{Et}_{2} \mathrm{O}$. The organic layer was washed with water and brine, and dried over $\mathrm{Na}_{2} \mathrm{SO}_{4}$. After concentration under reduced pressure, the residue was purified by chromatography over silica gel (Hexanes:EtOAc, 9:1) to give the desired compound (79\%) as a colorless viscous oil.

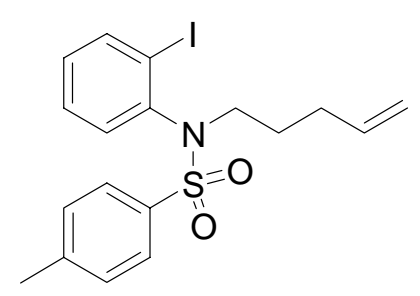

$N$-(2-Iodo-phenyl)-4-methyl- $N$-pent-4-enyl-benzenesulfonamid e: colorless viscous oil, purified by chromatography over silica gel (79\%, Hexanes:EtOAc, 9:1, Rf = 0.3); ${ }^{1} \mathrm{H} \mathrm{NMR}\left(\mathrm{CDCl}_{3}, 400 \mathrm{MHz}\right)$ $7.90(\mathrm{dd}, J=8.1 \mathrm{~Hz}, J=1.5 \mathrm{~Hz}, 1 \mathrm{H}), 7.61(\mathrm{~d}, J=8.1 \mathrm{~Hz}, 2 \mathrm{H})$, 7.28-7.25 (m, 3H), $7.01(\mathrm{dt}, J=7.3 \mathrm{~Hz}, J=1.5 \mathrm{~Hz}, 1 \mathrm{H}), 6.88(\mathrm{dd}, J$ $=8.1 \mathrm{~Hz}, J=1.5 \mathrm{~Hz}, 1 \mathrm{H}), 5.75-5.64(\mathrm{tdd}, J=17.2 \mathrm{~Hz}, J=10.2 \mathrm{~Hz}, J=7.0 \mathrm{~Hz}, 1 \mathrm{H}), 4.98-4.90$ (m, 2H), 3.71-3.64 (m, 1H), 3.38-3.30 (m, 1H), $2.41(\mathrm{~s}, 3 \mathrm{H}), 2.13-1.97(\mathrm{~m}, 2 \mathrm{H}), 1.71-1.44(\mathrm{~m}$, $2 \mathrm{H}) ;{ }^{13} \mathrm{C}$ NMR $\left(\mathrm{CDCl}_{3}, 100 \mathrm{MHz}\right) 143.43,141.48,140.19,137.06,135.68,129.74,129.63$, 129.27, 128.62, 127.84, 115.05, 103.19, 51.20, 30.66, 27.09, 21.35; IR (film) 3061.4, 2924.6, 
2858.1, 1639.8, 1596.8, 1493.9, 1466.1, 1436.0, 1350.9, 1164.4, 1091.0, 1018.5, 916.4, 815.1, 767.5, 715.0, $657.8 \mathrm{~cm}^{-1}$; HRMS-ESI (m/z): $[\mathrm{M}+\mathrm{H}]^{+}$calcd for $\mathrm{C}_{18} \mathrm{H}_{21} \mathrm{NO}_{2} \mathrm{SI} 442.0332$, found 442.0339 .
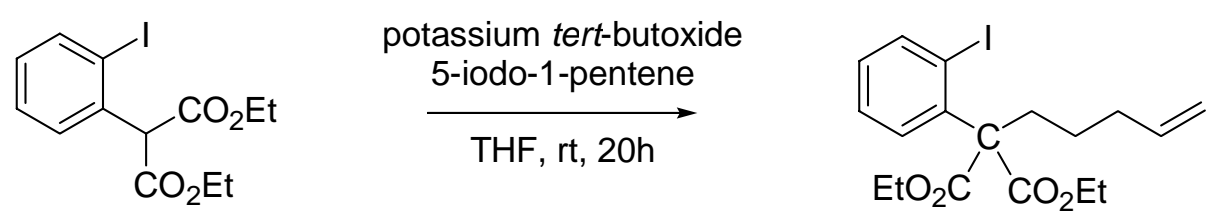

To a solution of 2-(2-iodophenyl)-malonic acid diethyl ester (1 eq.) in THF (15 mL) were added potassium tert-butoxide (1.3 eq.) and 5-iodo-1-pentene (1.2 eq.) (5-iodo-1-pentene was prepared from 5-bromo-1-pentene according to a procedure described in the literature ${ }^{3}$ ). After stirring at room temperature for $20 \mathrm{~h}$, the mixture was quenched with water. The aqueous layer was extracted with $\mathrm{Et}_{2} \mathrm{O}$. The organic layer was washed with water and brine and dried over $\mathrm{Na}_{2} \mathrm{SO}_{4}$. After concentration under reduced pressure, the residue was purified by chromatography over silica gel (Hexanes:EtOAc, 9:1) to give the desired compound $(81 \%)$ as a colorless oil.

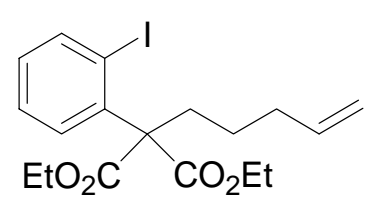

2-(2-Iodo-phenyl)-2-pent-4-enyl-malonic acid diethyl ester: colorless viscous oil, purified by chromatography over silica gel (81\%, Hexanes:EtOAc, 9:1, $\mathrm{Rf}=0.4) ;{ }^{1} \mathrm{H} \mathrm{NMR}\left(\mathrm{CDCl}_{3}, 400 \mathrm{MHz}\right) 7.92(\mathrm{~d}$, $J=7.7 \mathrm{~Hz}, 1 \mathrm{H}), 7.30(\mathrm{t}, J=7.3 \mathrm{~Hz}, 1 \mathrm{H}), 7.09(\mathrm{~d}, J=8.1 \mathrm{~Hz}, 1 \mathrm{H}), 6.93$ (t, $J=7.3 \mathrm{~Hz}, 1 \mathrm{H}), 5.79$ (tdd, $J=16.8 \mathrm{~Hz}, J=10.2 \mathrm{~Hz}, J=6.6 \mathrm{~Hz}, 1 \mathrm{H}), 4.97(\mathrm{~d}, J=16.8 \mathrm{~Hz}$, $1 \mathrm{H}), 4.91(\mathrm{~d}, J=10.2 \mathrm{~Hz}, 1 \mathrm{H}), 4.33-4.17(\mathrm{~m}, 4 \mathrm{H}), 2.49-2.45(\mathrm{~m}, 2 \mathrm{H}), 2.07-2.01(\mathrm{~m}, 2 \mathrm{H})$, 1.29-1.20 (m, 8H); ${ }^{13} \mathrm{C}$ NMR $\left(\mathrm{CDCl}_{3}, 100 \mathrm{MHz}\right) 170.13,142.01,140.19,138.31,129.95$, 128.63, 127.80, 114.79, 98.00, 66.38, 61.99, 33.98, 33.74, 24.74, 13.85; IR (film) 2979.2, 2932.0, 2902.7, 2867.5, 1744.8, 1731.2, 1465.4, 1439.2, 1363.2, 1296.1, 1254.2, 1196.5, 1135.4, 1095.7, 1009.8, 912.7, 857.9, $744.5 \mathrm{~cm}^{-1}$; HRMS-ESI $(\mathrm{m} / \mathrm{z}):[\mathrm{M}+\mathrm{H}]^{+}$calcd for $\mathrm{C}_{18} \mathrm{H}_{24} \mathrm{O}_{4} \mathrm{I} 431.0724$, found 431.0712 .

${ }^{3}$ Shi, B.; Hawryluk, N. A.; Snider B. B. J. Org. Chem. 2003, 68, 1030. 

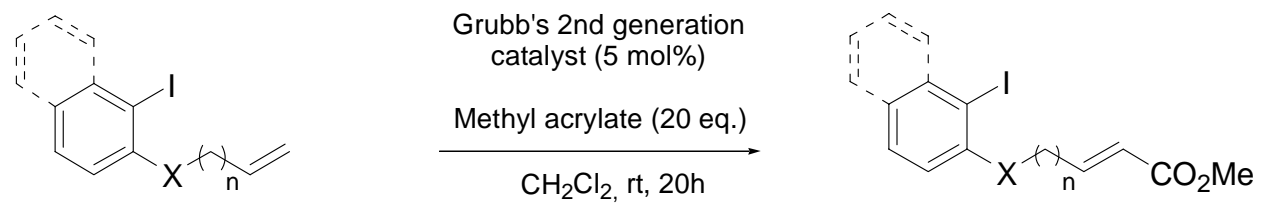

$\mathrm{X}=\mathrm{O}, \mathrm{NTs}, \mathrm{C}\left(\mathrm{CO}_{2} \mathrm{Et}\right)_{2}$

$\mathrm{n}=2,3$

To a solution of substrate (1 eq.) and methyl acrylate (20 eq.) in $\mathrm{CH}_{2} \mathrm{Cl}_{2}$ (2 mL/1 mmol of substrate) was added Grubb's $2^{\text {nd }}$ generation catalyst $(5 \mathrm{~mol} \%)$. The resulting mixture was stirred at room temperature for 20h and DMSO (50 eq. relative to the Grubb's catalyst) was added and the mixture was stirred for further $15 \mathrm{~h}$. Evaporation of the volatiles gave residue that was purified over silica gel using mixtures of Hexanes:EtOAc as eluent.

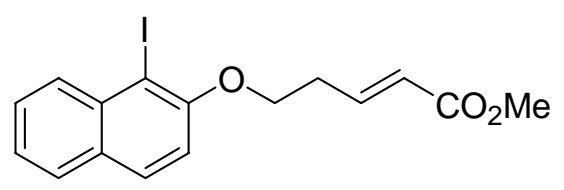

5-(1-Iodo-naphthalen-2-yloxy)-pent-2-enoic acid methyl ester: white solid, purified by chromatography over silica gel (67\%, Hexanes:EtOAc, 9:1, Rf = 0.2); M.p. 61-63 ${ }^{\circ} \mathrm{C} ;{ }^{1} \mathrm{H} \mathrm{NMR}\left(\mathrm{CDCl}_{3}, 400 \mathrm{MHz}\right) 8.13(\mathrm{~d}, J=8.4 \mathrm{~Hz}$, $1 \mathrm{H}), 7.73(\mathrm{~d}, J=8.8 \mathrm{~Hz}, 1 \mathrm{H}), 7.70(\mathrm{~d}, J=8.1 \mathrm{~Hz}, 1 \mathrm{H}), 7.51(\mathrm{dd}, J=8.1 \mathrm{~Hz}, 1 \mathrm{H}), 7.36(\mathrm{t}, J=$ $8.1 \mathrm{~Hz}, 1 \mathrm{H}), 7.14(\mathrm{td}, J=15.7 \mathrm{~Hz}, J=7.0 \mathrm{~Hz}, 1 \mathrm{H}), 7.04(\mathrm{~d}, J=8.8 \mathrm{~Hz}, 1 \mathrm{H}), 6.04(\mathrm{~d}, J=15.7$ $\mathrm{Hz}, 1 \mathrm{H}), 4.16(\mathrm{t}, J=6.6 \mathrm{~Hz}, 2 \mathrm{H}), 3.74(\mathrm{~s}, 3 \mathrm{H}), 2.73-2.71(\mathrm{~m}, 2 \mathrm{H}) ;{ }^{13} \mathrm{C} \mathrm{NMR}\left(\mathrm{CDCl}_{3}, 100\right.$ MHz) 166.48, 155.47, 144.56, 135.41, 131.09, 130.12, 129.85, 128.00, 127.92, 124.35, 123.25, 113.98, 88.69, 68.14, 51.36, 32.12; IR (film) 2943.7, 2873.4, 2832.3, 1721.9, 1620.3, 1591.0, 1503.5, 1456.7, 1433.3, 1346.3, 1325.3, 1266.0, 1237.6, 1167.5, 1056.0, 802.5 $\mathrm{cm}^{-1}$; HRMS-ESI (m/z): $[\mathrm{M}+\mathrm{H}]^{+}$calcd for $\mathrm{C}_{16} \mathrm{H}_{16} \mathrm{O}_{3} \mathrm{I} 383.0138$, found 383.0158 .

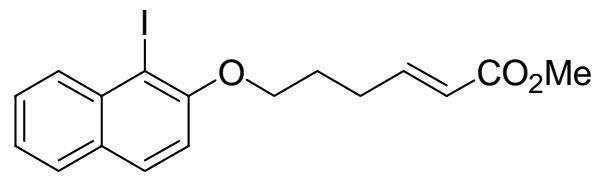

6-(1-Iodo-naphthalen-2-yloxy)-hex-2-enoic acid methyl ester: colorless viscous oil, purified by chromatography over silica gel $(62 \%$, Hexanes:EtOAc, 9:1, Rf = 0.2); ${ }^{1} \mathrm{H} \mathrm{NMR}\left(\mathrm{CDCl}_{3}, 400 \mathrm{MHz}\right) 8.13(\mathrm{~d}, J=8.4 \mathrm{~Hz}, 1 \mathrm{H}), 7.70(\mathrm{~m}, 2 \mathrm{H}), 7.51$ (ddd, $J=8.4 \mathrm{~Hz}, J=7.0 \mathrm{~Hz}, J=1.1 \mathrm{~Hz}, 1 \mathrm{H}), 7.35(\mathrm{ddd}, J=8.1 \mathrm{~Hz}, J=7.0 \mathrm{~Hz}, J=1.1 \mathrm{~Hz}, 1 \mathrm{H})$, $7.05(\mathrm{td}, J=15.8 \mathrm{~Hz}, J=7.0 \mathrm{~Hz}, 1 \mathrm{H}), 7.03(\mathrm{~d}, J=8.8 \mathrm{~Hz}, 1 \mathrm{H}), 5.92(\mathrm{td}, J=15.8 \mathrm{~Hz}, J=1.5$ $\mathrm{Hz}, 1 \mathrm{H}), 4.05(\mathrm{t}, J=5.9 \mathrm{~Hz}, 2 \mathrm{H}), 3.72(\mathrm{~s}, 3 \mathrm{H}), 2.53-2.47(\mathrm{~m}, 2 \mathrm{H}), 1.99-1.93(\mathrm{~m}, 2 \mathrm{H}) ;{ }^{13} \mathrm{C}$ $\mathrm{NMR}\left(\mathrm{CDCl}_{3}, 100 \mathrm{MHz}\right) 166.61,155.55,148.04,135.33,130.90,130.02,129.67,127.94$, 
127.83, 124.13, 121.43, 113.76, 88.24, 68.57, 51.18, 28.60, 27.62; IR (film) 2947.6, 2879.0, 2845.0, 1721.1, 1658.8, 1621.4, 1593.2, 1555.5, 1501.5, 1458.1, 1434.6, 1346.0, 1327.8, 1266.0, 1242.0, 1206.7, 1166.8, 1061.4, 978.8, 803.3, 764.0, 747.0 $\mathrm{cm}^{-1}$; HRMS-EI (m/z): $\left[\mathrm{M}^{+}\right]$calcd for $\mathrm{C}_{17} \mathrm{H}_{17} \mathrm{O}_{3} \mathrm{I} 396.022247$, found 396.022800 .

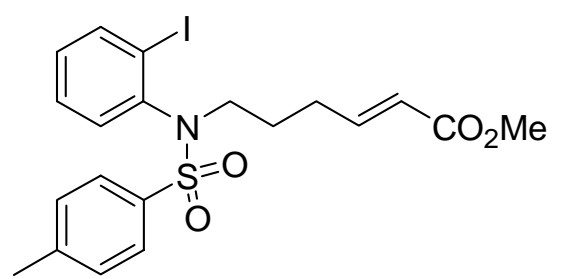

6-[(2-Iodo-phenyl)-(toluene-4-sulfonyl)-amino]-hex-2-
enoic acid methyl ester: colorless viscous oil, purified by
chromatography over silica gel $(80 \%$, Hexanes:EtOAc,
$8: 2, \mathrm{Rf}=0.3) ;{ }^{1} \mathrm{H} \mathrm{NMR}\left(\mathrm{CDCl}_{3}, 400 \mathrm{MHz}\right) 7.92(\mathrm{dd}, J=$
$7.9 \mathrm{~Hz}, J=1.5 \mathrm{~Hz}, 1 \mathrm{H}), 7.61(\mathrm{~d}, J=8.2 \mathrm{~Hz}, 2 \mathrm{H}), 7.32-7.26$

$(\mathrm{m}, 3 \mathrm{H}), 7.03(\mathrm{dt}, J=7.9 \mathrm{~Hz}, J=1.5 \mathrm{~Hz}, 1 \mathrm{H}), 6.89(\mathrm{dd}, J=7.9 \mathrm{~Hz}, J=1.8 \mathrm{~Hz}, 1 \mathrm{H}), 6.86(\mathrm{td}$, $J=15.5 \mathrm{~Hz}, J=7.0 \mathrm{~Hz}, 1 \mathrm{H}), 5.77(\mathrm{td}, J=15.5 \mathrm{~Hz}, J=1.5 \mathrm{~Hz}, 1 \mathrm{H}), 3.73-3.64(\mathrm{~m}, 1 \mathrm{H}), 3.70$ (s, 3H), 3.40-3.31 (m, 1H), $2.43(\mathrm{~s}, 3 \mathrm{H}), 2.36-2.18(\mathrm{~m}, 2 \mathrm{H}), 1.77-1.52(\mathrm{~m}, 2 \mathrm{H}) ;{ }^{13} \mathrm{C}$ NMR $\left(\mathrm{CDCl}_{3}, 100 \mathrm{MHz}\right)$ 166.63, 147.61, 143.67, 141.43, 140.40, 135.58, 129.82, 129.39, 128.77, 127.96, 121.40, 103.13, 51.25, 51.17, 29.32, 26.46, 21.41; IR (film) 2941.9, 2860.0, 1720.1, 1656.0, 1592.2, 1466.1, 1435.7, 1349.8, 1273.0, 1201.4, 1160.5, 1089.7, 1037.2, 1019.2, 815.5, 714.7, $657.6 \mathrm{~cm}^{-1}$; HRMS-ESI (m/z): $[\mathrm{M}+\mathrm{Na}]^{+}$calcd for $\mathrm{C}_{20} \mathrm{H}_{22} \mathrm{NO}_{4} \mathrm{SINa} 522.0206$, found 522.0181 .

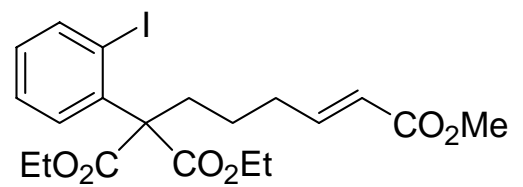

7-Ethoxycarbonyl-7-(2-iodo-phenyl)-oct-2-enedioic acid 8-ethyl ester 1-methyl ester: colorless viscous oil, purified by chromatography over silica gel $(65 \%$, Hexanes:EtOAc, 8:2, Rf $=0.45) ;{ }^{1} \mathrm{H}$ NMR $\left(\mathrm{CDCl}_{3}, 400 \mathrm{MHz}\right) 7.93(\mathrm{dd}, J=8.1 \mathrm{~Hz}, J=1.5 \mathrm{~Hz}, 1 \mathrm{H}), 7.31(\mathrm{td}, J$ $=7.7 \mathrm{~Hz}, J=1.5 \mathrm{~Hz}, 1 \mathrm{H}), 7.06(\mathrm{dd}, J=8.1 \mathrm{~Hz}, J=1.5 \mathrm{~Hz}, 1 \mathrm{H}), 6.95(\mathrm{td}, J=7.7 \mathrm{~Hz}, J=1.5$ $\mathrm{Hz}, 1 \mathrm{H}), 6.90(\mathrm{td}, J=15.8 \mathrm{~Hz}, J=7.0 \mathrm{~Hz}, 1 \mathrm{H}), 5.79(\mathrm{td}, J=15.8 \mathrm{~Hz}, J=1.5 \mathrm{~Hz}, 1 \mathrm{H})$, 4.35-4.19 (m, 4H), 3.69 (s, 3H), 2.53-2.49 (m, 2H), 2.22-2.17 (m, 2H), 1.35-1.25 (m, 2H), $1.27(\mathrm{t}, J=7.3 \mathrm{~Hz}, 6 \mathrm{H}),{ }^{13} \mathrm{C} \mathrm{NMR}\left(\mathrm{CDCl}_{3}, 100 \mathrm{MHz}\right) 169.80,166.72,148.63,141.91,139.64$, 129.84, 128.59, 127.70, 121.01, 97.50, 66.19, 61.87, 51.11, 33.24, 32.16, 23.81, 13.65; IR (film) 2980.0, 2947.1, 2897.8, 1740.6, 1724.4, 1658.0, 1465.2, 1435.5, 1366.5, 1254.1, 1196.3, 1153.6, 1096.3, 1010.0, 978.9, 859.7, $744.5 \mathrm{~cm}^{-1}$; HRMS-ESI (m/z): $[\mathrm{M}+\mathrm{H}]^{+}$calcd for $\mathrm{C}_{20} \mathrm{H}_{26} \mathrm{O}_{6} \mathrm{I}$ 489.0768, found 489.0757 . 


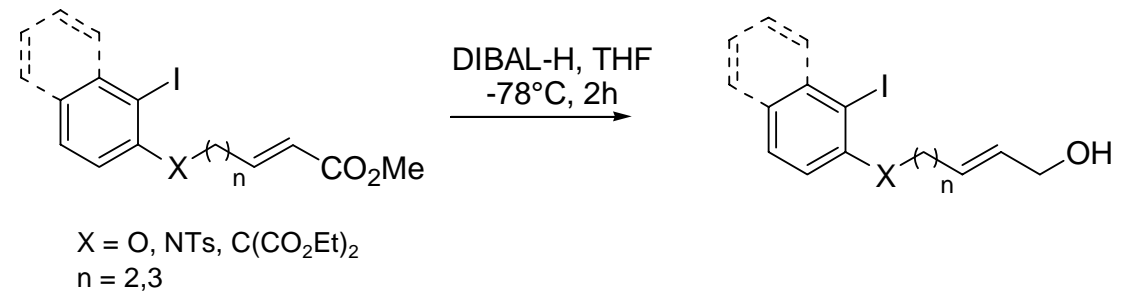

To a solution of substrate ( 1 eq. $)$ in THF $(\mathrm{C}=0.2 \mathrm{M})$ cooled at $-78{ }^{\circ} \mathrm{C}$ was added DIBAL-H $1.5 \mathrm{M}$ in toluene (2.5 eq.). After stirring at $-78{ }^{\circ} \mathrm{C}$ for $2 \mathrm{~h}$, the mixture was allowed to warm at room temperature. The reaction was quenched with a $20 \%$ solution of $\mathrm{K} / \mathrm{Na}$ tartrate and stirred for few minutes before being extracted with $\mathrm{Et}_{2} \mathrm{O}$. The combined organic layers were washed with water, brine and dried over $\mathrm{Na}_{2} \mathrm{SO}_{4}$. After concentration under reduced pressure, the residue was purified by chromatography over silica gel using mixtures of hexanes/EtOAc as eluent to give the desired compounds as colorless viscous oils.

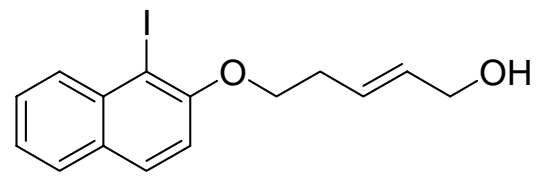

5-(1-Iodo-naphthalen-2-yloxy)-pent-2-en-1-ol: colorless viscous oil, purified by chromatography over silica gel (78\%, Hexanes:EtOAc, 8:2, Rf $=0.2) ;{ }^{1} \mathrm{H}$ NMR $\left(\mathrm{CDCl}_{3}\right.$, $400 \mathrm{MHz}) 8.13(\mathrm{~d}, J=8.4 \mathrm{~Hz}, 1 \mathrm{H}), 7.76(\mathrm{~d}, J=8.8 \mathrm{~Hz}, 1 \mathrm{H}), 7.71(\mathrm{~d}, J=8.1 \mathrm{~Hz}, 1 \mathrm{H}), 7.52(\mathrm{t}$, $J=8.1 \mathrm{~Hz}, 1 \mathrm{H}), 7.37(\mathrm{t}, J=7.7 \mathrm{~Hz}, 1 \mathrm{H}), 7.12(\mathrm{~d}, J=8.8 \mathrm{~Hz}, 1 \mathrm{H}), 5.92-5.79(\mathrm{~m}, 2 \mathrm{H})$, 4.18-4.12 (m, 4H), 2.64-2.59 (m, 2H), $1.70(\mathrm{~s}, \mathrm{OH}) ;{ }^{13} \mathrm{C} \mathrm{NMR}\left(\mathrm{CDCl}_{3}, 100 \mathrm{MHz}\right) 155.84$, 135.36, 131.91, 131.16, 130.18, 129.89, 128.10, 128.08, 127.99, 124.33, 114.20, 88.66, 69.54, 63.46, 32.32; IR (film) 3363.9, 2927.2, 2873.3, 1621.1, 1592.6, 1500.9, 1457.6, 1426.3, 1345.5, 1328.6, 1266.1, 1241.1, 1148.9, 1057.3, 1022.8, 971.0, $801.8 \mathrm{~cm}^{-1}$; HRMS-ESI (m/z): $[\mathrm{M}+\mathrm{Na}]^{+}$calcd for $\mathrm{C}_{15} \mathrm{H}_{15} \mathrm{O}_{2} \mathrm{INa} 377.0009$, found 376.9997 .

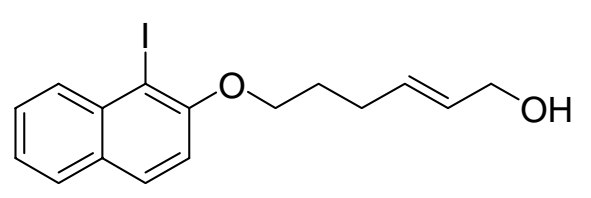

6-(1-Iodo-naphthalen-2-yloxy)-hex-2-en-1-ol: colorless viscous oil, purified by chromatography over silica gel (95\%, Hexanes:EtOAc, 8:2, Rf = 0.2); ${ }^{1} \mathrm{H}$ NMR $\left(\mathrm{CDCl}_{3}, 400 \mathrm{MHz}\right) 8.13(\mathrm{~d}, J=8.4 \mathrm{~Hz}, 1 \mathrm{H}), 7.78(\mathrm{~d}, J=$ $8.8 \mathrm{~Hz}, 1 \mathrm{H}), 7.72(\mathrm{~d}, J=8.1 \mathrm{~Hz}, 1 \mathrm{H}), 7.53(\mathrm{ddd}, J=8.4 \mathrm{~Hz}, J=7.0 \mathrm{~Hz}, J=1.1 \mathrm{~Hz}, 1 \mathrm{H}), 7.37$ (ddd, $J=8.1 \mathrm{~Hz}, J=7.0 \mathrm{~Hz}, J=1.1 \mathrm{~Hz}, 1 \mathrm{H}), 7.15(\mathrm{~d}, J=8.8 \mathrm{~Hz}, 1 \mathrm{H}), 5.82-5.69(\mathrm{~m}, 2 \mathrm{H}), 4.17$ $(\mathrm{t}, J=6.2 \mathrm{~Hz}, 2 \mathrm{H}), 4.09(\mathrm{~d}, J=4.8 \mathrm{~Hz}, 2 \mathrm{H}), 2.40-2.35(\mathrm{~m}, 2 \mathrm{H}), 2.01-1.94(\mathrm{~m}, 2 \mathrm{H}), 1.37(\mathrm{~s}$, $\mathrm{OH}) ;{ }^{13} \mathrm{C} \mathrm{NMR}\left(\mathrm{CDCl}_{3}, 100 \mathrm{MHz}\right) 155.78,135.40,131.41,130.95,130.03,129.72,129.67$, 
127.98, 127.83, 124.10, 113.95, 88.28, 69.08, 63.22, 28.64, 28.57; IR (film) 3354.8, 2937.9, 2863.4, 1621.3, 1592.9, 1500.6, 1457.3, 1426.8, 1345.6, 1328.5, 1264.7, 1241.8, 1148.8, 1058.8, 1020.7, 969.4, 802.0, 763.2, $745.8 \mathrm{~cm}^{-1}$; HRMS-ESI $(\mathrm{m} / \mathrm{z}):[\mathrm{M}+\mathrm{Na}]^{+}$calcd for $\mathrm{C}_{16} \mathrm{H}_{17} \mathrm{O}_{2} \mathrm{INa} 391.0165$, found 391.0157 .

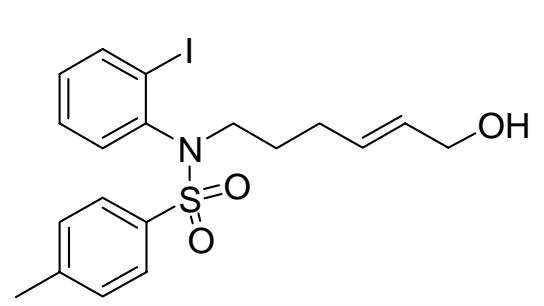

$N$-(6-Hydroxy-hex-4-enyl)- $N$-(2-iodo-phenyl)-4-methylbenzenesulfonamide: colorless viscous oil, purified by chromatography over silica gel (88\%, Hexanes:EtOAc, 7:3, $\mathrm{Rf}=0.2) ;{ }^{1} \mathrm{H} \mathrm{NMR}\left(\mathrm{CDCl}_{3}, 400 \mathrm{MHz}\right) 7.91(\mathrm{~d}, J=8.1 \mathrm{~Hz}$, $1 \mathrm{H}), 7.61(\mathrm{~d}, J=8.1 \mathrm{~Hz}, 2 \mathrm{H}), 7.29-7.25(\mathrm{~m}, 3 \mathrm{H}), 7.02(\mathrm{t}, J=$ $7.7 \mathrm{~Hz}, 1 \mathrm{H}), 6.89(\mathrm{~d}, J=7.7 \mathrm{~Hz}, 1 \mathrm{H}), 5.62-5.52(\mathrm{~m}, 2 \mathrm{H})$, 4.06-3.97 (m, 2H), 3.70-3.63 (m, 1H), 3.37-3.30 (m, 1H), $2.43(\mathrm{~s}, 3 \mathrm{H}), 2.14-1.95(\mathrm{~m}, 3 \mathrm{H})$, 1.68-1.58 (m, 1H), 1.53-1.43 (m, 1H); ${ }^{13} \mathrm{C}$ NMR $\left(\mathrm{CDCl}_{3}, 100 \mathrm{MHz}\right)$ 143.57, 141.43, 140.32, 135.64, 130.89, 129.85, 129.72, 129.35, 128.68, 127.94, 103.16, 63.14, 51.18, 29.21, 27.39, 21.41; IR (film) 3372.6, 3055.3, 2926.5, 1596.8, 1466.1, 1435.9, 1348.3, 1160.0, 1090.3, 1018.4, 971.2, 815.6, $767.8 \mathrm{~cm}^{-1}$; HRMS-ESI (m/z): $[\mathrm{M}+\mathrm{Na}]^{+}$calcd for $\mathrm{C}_{19} \mathrm{H}_{22} \mathrm{NO}_{3} \mathrm{SINa}$ 494.0257, found 494.0249 .

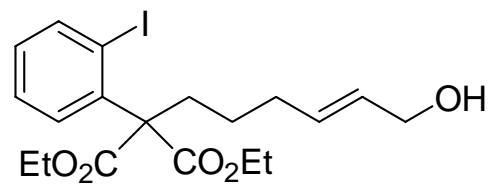

2-(6-Hydroxy-hex-4-enyl)-2-(2-iodo-phenyl)-malonic acid diethyl ester: colorless viscous oil, purified by chromatography over silica gel (98\%, Hexanes:EtOAc, 7:3, $\mathrm{Rf}=0.3) ;{ }^{1} \mathrm{H} \mathrm{NMR}\left(\mathrm{CDCl}_{3}, 400 \mathrm{MHz}\right) 7.88(\mathrm{~d}, J=7.7 \mathrm{~Hz}, 1 \mathrm{H}), 7.26(\mathrm{t}, J=8.1 \mathrm{~Hz}, 1 \mathrm{H}), 7.04$ $(\mathrm{d}, J=8.1 \mathrm{~Hz}, 1 \mathrm{H}), 6.89(\mathrm{t}, J=7.7 \mathrm{~Hz}, 1 \mathrm{H}), 5.60-5.50(\mathrm{~m}, 2 \mathrm{H}), 4.29-4.13(\mathrm{~m}, 4 \mathrm{H}), 3.98-3.97$ (m, 2H), 2.45-2.41 (m, 2H), 2.01-1.97 (m, 2H), $1.89(\mathrm{~s}, \mathrm{OH}), 1.28-1.15(\mathrm{~m}, 2 \mathrm{H}), 1.22(\mathrm{t}, J=$ $7.3 \mathrm{~Hz}, 6 \mathrm{H}) ;{ }^{13} \mathrm{C}$ NMR $\left(\mathrm{CDCl}_{3}, 100 \mathrm{MHz}\right) 169.96,141.86,139.93,132.11,129.83,129.38$, 128.52, 127.67, 97.76, 66.25, 63.37, 61.86, 33.52, 32.20, 24.80, 13.69; IR (film) 3340.1, 2979.2, 2938.6, 2899.9, 2866.8, 1715.7, 1651.7, 1464.1, 1442.0, 1389.8, 1365.0, 1251.9, 1191.9, 1161.6, 1093.3, 1008.1, 969.2, $856.6 \mathrm{~cm}^{-1}$; HRMS-ESI $(\mathrm{m} / \mathrm{z}):[\mathrm{M}+\mathrm{H}]^{+}$calcd for $\mathrm{C}_{19} \mathrm{H}_{26} \mathrm{O}_{5} \mathrm{I} 461.0819$, found 461.0842 . 

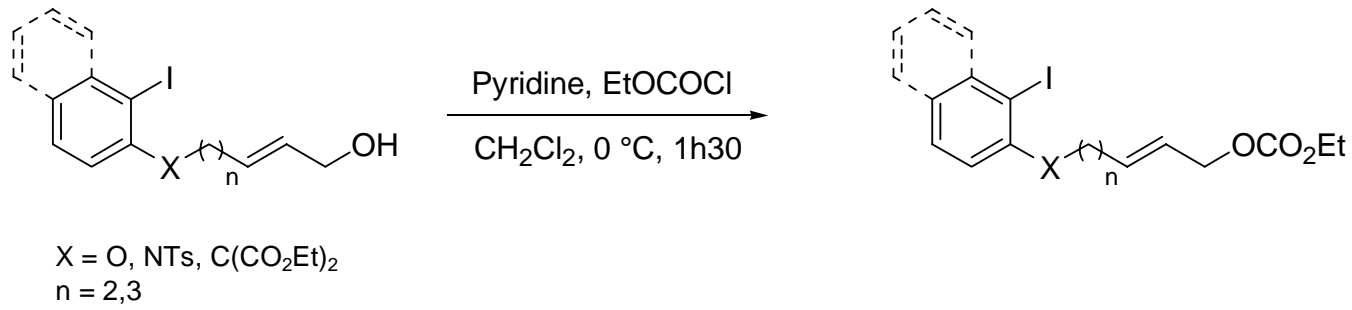

To a solution of the substrate ( 1 eq.) in $\mathrm{CH}_{2} \mathrm{Cl}_{2}(\mathrm{C}=0.6 \mathrm{M})$ were successively added pyridine (1.5 eq.) and ethyl chloroformate ( 1.2 eq.) at $0{ }^{\circ} \mathrm{C}$. The resulting mixture was stirred for $1 \mathrm{~h} 30$ at $0{ }^{\circ} \mathrm{C}$. Then, the reaction mixture was diluted with $\mathrm{CH}_{2} \mathrm{Cl}_{2}$ and washed with $1 \mathrm{~N} \mathrm{HCl}$, saturated $\mathrm{NaHCO}_{3}$ and brine. The organic layer was dried over $\mathrm{Na}_{2} \mathrm{SO}_{4}$ and concentrated under reduced pressure. The residue was purified by chromatography over silica gel using mixtures of hexanes/EtOAc as eluent.<smiles>CCOOC/C=C/CCOc1ccc2ccccc2c1I</smiles>

$\begin{array}{lll}\text { Carbonic acid ethyl } & \text { ester } \\ \text { 5-(1-iodo-naphthalen-2-yloxy)-pent-2-enyl } & \text { ester } \\ \text { (4a): white solid, purified by chromatography } & \text { over }\end{array}$ silica gel (87\%, Hexanes:EtOAc, 9:1, Rf = 0.26); M.p. 66-68 ${ }^{\circ} \mathrm{C} ;{ }^{1} \mathrm{H}$ NMR $\left(\mathrm{CDCl}_{3}, 400\right.$ MHz) $8.13(\mathrm{~d}, J=8.4 \mathrm{~Hz}, 1 \mathrm{H}), 7.78(\mathrm{~d}, J=8.8 \mathrm{~Hz}, 1 \mathrm{H}), 7.72(\mathrm{~d}, J=8.1 \mathrm{~Hz}, 1 \mathrm{H}), 7.52$ (ddd, $J=8.4 \mathrm{~Hz}, J=7.0 \mathrm{~Hz}, J=1.1 \mathrm{~Hz}, 1 \mathrm{H}), 7.37(\mathrm{ddd}, J=8.1 \mathrm{~Hz}, J=7.0 \mathrm{~Hz}, J=1.1 \mathrm{~Hz}, 1 \mathrm{H})$, $7.14(\mathrm{~d}, J=8.8 \mathrm{~Hz}, 1 \mathrm{H}), 6.06-5.99(\mathrm{~m}, 1 \mathrm{H}), 5.85-5.77(\mathrm{~m}, 1 \mathrm{H}), 4.62(\mathrm{t}, J=6.2 \mathrm{~Hz}, 2 \mathrm{H}), 4.19$ (m, 4H), 2.68-2.63 (m, 2H), $1.30(\mathrm{t}, J=7.3 \mathrm{~Hz}, 3 \mathrm{H}) ;{ }^{13} \mathrm{C} \mathrm{NMR}\left(\mathrm{CDCl}_{3}, 100 \mathrm{MHz}\right) 155.92$, 154.86, 135.49, 131.96, 131.11, 130.10, 129.83, 128.02, 127.91, 126.18, 124.27, 114.10, 88.59, 69.14, 67.91, 63.81, 32.29, 14.18; IR (film) 3058.0, 2981.2, 2935.1, 2873.7, 1743.1, 1621.3, 1593.1, 1502.7, 1458.2, 1427.1, 1380.8, 1346.0, 1257.9, 1149.5, 1056.7, 1023.0, 973.7, 871.9, 802.2, 764.0, $747.0 \mathrm{~cm}^{-1}$; HRMS-ESI (m/z): $[\mathrm{M}+\mathrm{Na}]^{+}$calcd for $\mathrm{C}_{18} \mathrm{H}_{19} \mathrm{O}_{4} \mathrm{INa}$ 449.0220, found 449.0217 . 


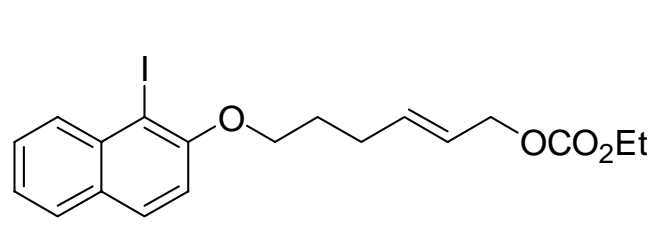

Carbonic acid ethyl ester 6-(1-iodo-naphthalen-2-yloxy)-hex-2-enyl ester (4b): white solid, purified by chromatography over silica gel (94\%, Hexanes:EtOAc, 9:1, Rf = 0.3); M.p. 40-42 ${ }^{\circ} \mathrm{C} ;{ }^{1} \mathrm{H} \mathrm{NMR}\left(\mathrm{CDCl}_{3}, 400 \mathrm{MHz}\right) 8.13(\mathrm{~d}, J=8.4 \mathrm{~Hz}, 1 \mathrm{H}), 7.76(\mathrm{~d}, J=8.8 \mathrm{~Hz}, 1 \mathrm{H}), 7.71$ $(\mathrm{d}, J=8.1 \mathrm{~Hz}, 1 \mathrm{H}), 7.52(\mathrm{ddd}, J=8.4 \mathrm{~Hz}, J=7.0 \mathrm{~Hz}, J=1.1 \mathrm{~Hz}, 1 \mathrm{H}), 7.36(\mathrm{ddd}, J=8.1 \mathrm{~Hz}$, $J=7.0 \mathrm{~Hz}, J=1.1 \mathrm{~Hz}, 1 \mathrm{H}), 7.12(\mathrm{~d}, J=8.8 \mathrm{~Hz}, 1 \mathrm{H}), 5.93-5.86(\mathrm{~m}, 1 \mathrm{H}), 5.73-5.65(\mathrm{~m}, 1 \mathrm{H})$, $4.58(\mathrm{~d}, J=6.6 \mathrm{~Hz}, 2 \mathrm{H}), 4.18(\mathrm{q}, J=7.7 \mathrm{~Hz}, 2 \mathrm{H}), 4.13(\mathrm{t}, J=5.9 \mathrm{~Hz}, 2 \mathrm{H}), 2.42-2.36(\mathrm{~m}, 2 \mathrm{H})$, 2.00-1.95 (m, 2H), $1.29(\mathrm{t}, J=7.7 \mathrm{~Hz}, 3 \mathrm{H}) ;{ }^{13} \mathrm{C} \mathrm{NMR}\left(\mathrm{CDCl}_{3}, 100 \mathrm{MHz}\right) 155.88,154.90$, $135.68,135.54,131.10,130.11,129.81,128.05,127.92,124.22,114.05,88.41,69.08,68.10$, 63.80, 28.72, 28.47, 14.19; IR (film) 3051.8, 2977.9, 2942.1, 2875.5, 1743.3, 1621.6, 1593.3, 1502.1, 1458.2, 1346.0, 1259.0, 1149.4, 1060.0, 1017.8, 973.0, 873.6, 802.9, $746.9 \mathrm{~cm}^{-1}$; HRMS-ESI (m/z): $[\mathrm{M}+\mathrm{Na}]^{+}$calcd for $\mathrm{C}_{19} \mathrm{H}_{21} \mathrm{O}_{4} \mathrm{INa} 463.0376$, found 463.0353 .

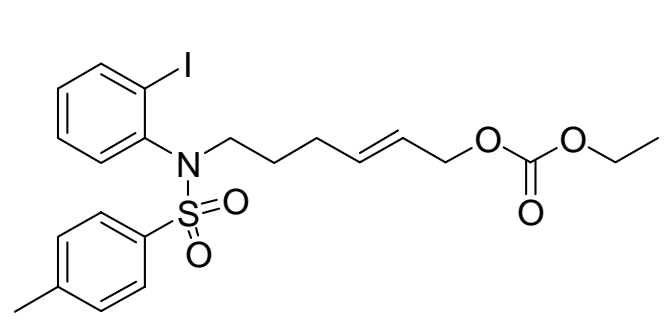

Carbonic acid ethyl ester 6-[(2-iodo-phenyl)-(toluene-4-sulfonyl)-amino]hex-2-enyl ester (4c): colorless viscous oil, purified by chromatography over silica gel $(90 \%$, Hexanes:EtOAc, 8:2, $\mathrm{Rf}=0.3) ;{ }^{1} \mathrm{H}$ NMR $\left(\mathrm{CDCl}_{3}\right.$, $400 \mathrm{MHz}) 7.92(\mathrm{~d}, J=7.7 \mathrm{~Hz}, 1 \mathrm{H}), 7.63(\mathrm{~d}, J=8.1$

$\mathrm{Hz}, 2 \mathrm{H}), 7.30-7.27(\mathrm{~m}, 3 \mathrm{H}), 7.03(\mathrm{t}, J=7.3 \mathrm{~Hz}, 1 \mathrm{H}), 6.92(\mathrm{~d}, J=7.3 \mathrm{~Hz}, 1 \mathrm{H}), 5.74-5.67(\mathrm{~m}$, $1 \mathrm{H}), 5.57-5.50(\mathrm{~m}, 1 \mathrm{H}), 4.52(\mathrm{~d}, J=6.2 \mathrm{~Hz}, 2 \mathrm{H}), 4.18(\mathrm{q}, J=7.3 \mathrm{~Hz}, 2 \mathrm{H}), 3.68-3.61(\mathrm{~m}, 1 \mathrm{H})$, 3.38-3.31 (m, 1H), 2.44 (s, 3H), 2.16-2.00 (m, 2H), 1.70-1.45 (m, 2H), 1.30 (t, $J=7.3 \mathrm{~Hz}$, $3 \mathrm{H}) ;{ }^{13} \mathrm{C}$ NMR $\left(\mathrm{CDCl}_{3}, 100 \mathrm{MHz}\right) 154.89,143.62,141.61,140.49,135.24,135.94,130.13$, $129.80,129.44,128.76,128.12,124.20,103.15,68.02,63.86,51.29,29.41,27.30,21.52$, 14.22; IR (film) 2978.8, 2939.7, 2862.6, 1742.9, 1466.2, 1380.9, 1351.4, 1256.9, 1162.7, 1091.3, 1018.3, 715.2, $657.7 \mathrm{~cm}^{-1}$; HRMS-ESI (m/z): $[\mathrm{M}+\mathrm{Na}]^{+}$calcd for $\mathrm{C}_{22} \mathrm{H}_{26} \mathrm{NO}_{5} \mathrm{SINa}$ 566.0468 , found 566.0480 .

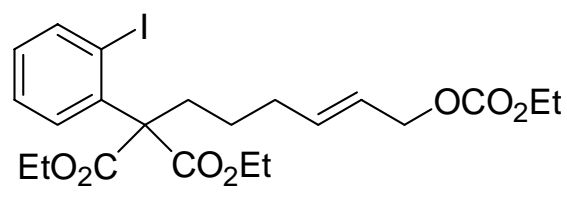

2-(6-Ethoxycarbonyloxy-hex-4-enyl)-2-(2-iodo-phenyl )-malonic acid diethyl ester (4d): colorless viscous oil, purified by chromatography over silica gel $(83 \%$, Hexanes:EtOAc, 8:2, $\mathrm{Rf}=0.45) ;{ }^{1} \mathrm{H}$ NMR $\left(\mathrm{CDCl}_{3}, 400 \mathrm{MHz}\right) 7.88(\mathrm{~d}, J=8.1 \mathrm{~Hz}, 1 \mathrm{H}), 7.27$ 
$(\mathrm{t}, J=7.7 \mathrm{~Hz}, 1 \mathrm{H}), 7.03(\mathrm{~d}, J=8.1 \mathrm{~Hz}, 1 \mathrm{H}), 6.90(\mathrm{t}, J=7.7 \mathrm{~Hz}, 1 \mathrm{H}), 5.70(\mathrm{td}, J=15.4 \mathrm{~Hz}, J$ $=6.6 \mathrm{~Hz}, 1 \mathrm{H}), 5.52(\mathrm{td}, J=15.4 \mathrm{~Hz}, J=6.6 \mathrm{~Hz}, 1 \mathrm{H}), 4.48(\mathrm{~d}, J=6.2 \mathrm{~Hz}, 2 \mathrm{H}), 4.30-4.17(\mathrm{~m}$, $4 \mathrm{H}), 4.13(\mathrm{q}, J=7.3 \mathrm{~Hz}, 2 \mathrm{H}), 2.46-2.42(\mathrm{~m}, 2 \mathrm{H}), 2.04-1.99(\mathrm{~m}, 2 \mathrm{H}), 1.26-1.17(\mathrm{~m}, 11 \mathrm{H}) ;{ }^{13} \mathrm{C}$ NMR $\left(\mathrm{CDCl}_{3}, 100 \mathrm{MHz}\right)$ 169.92, 154.85, 141.92, 139.92, 136.30, 129.86, 128.56, 127.71, $123.59,97.71,68.15,66.25,63.68,61.86,33.50,32.28,24.52,14.14,13.72$; IR (film) 2980.6, 2937.2, 2900.3, 1743.7, 1465.2, 1447.0, 1381.8, 1365.8, 1250.8, 1192.6, 1094.9, 1009.1, 971.4, 871.2, $744.2 \mathrm{~cm}^{-1}$; HRMS-ESI $(\mathrm{m} / \mathrm{z})$ : $[\mathrm{M}+\mathrm{Na}]^{+}$calcd for $\mathrm{C}_{22} \mathrm{H}_{29} \mathrm{O}_{7} \mathrm{INa}$ 555.0850 , found 555.0825 .
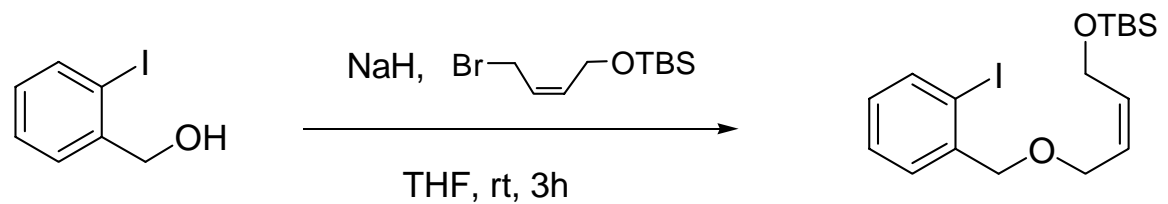

$95 \%$

To a suspension of $\mathrm{NaH} 80 \%$ in mineral oil $(0.12 \mathrm{~g})$ in dry THF $(12 \mathrm{~mL})$ was added the benzylic alcohol $(0.64 \mathrm{~g}, 2.7 \mathrm{mmol})$. After stirring for $10 \mathrm{~min}$ at room temperature, (Z) $-\mathrm{BrCH}_{2} \mathrm{CH}=\mathrm{CHCH}_{2} \mathrm{OTBS}(0.82 \mathrm{~g}, 2.9 \mathrm{mmol})$ was added and the resulting mixture was stirred for $3 \mathrm{~h}$ at room temperature. The reaction was carefully quenched with water. The aqueous layer was extracted with diethyl ether. The organic layer was dried over $\mathrm{Na}_{2} \mathrm{SO}_{4}$ and concentrated under reduced pressure. The residue was purified by chromatography over silica gel (Hexanes:EtOAc, 97:3) to give the desired compound (0.82 g, $95 \%)$ as a colorless viscous oil.

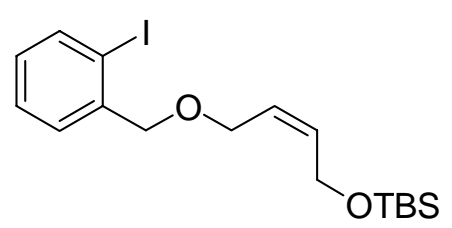

tert-Butyl-[4-(2-iodo-benzyloxy)-but-2-enyloxy]-dimethyl-sil ane: colorless viscous oil, purified by chromatography over silica gel (95\%, Hexanes:EtOAc, 97:3, Rf $=0.4) ;{ }^{1} \mathrm{H}$ NMR $\left(\mathrm{CDCl}_{3}, 400 \mathrm{MHz}\right) 7.82(\mathrm{~d}, J=7.7 \mathrm{~Hz}, 1 \mathrm{H}), 7.44(\mathrm{~d}, J=7.7 \mathrm{~Hz}$, 1H), 7.35 (t, $J=7.3 \mathrm{~Hz}, 1 \mathrm{H}), 6.99$ (t, $J=7.3 \mathrm{~Hz}, 1 \mathrm{H}), 5.77(\mathrm{~m}, 2 \mathrm{H}), 4.49$ (s, 2H), 4.27 (d, $J=$ $5.5 \mathrm{~Hz}, 2 \mathrm{H}), 4.17$ (d, J = 5.8 Hz, 2H), $0.90(\mathrm{~s}, 9 \mathrm{H}), 0.07$ (s, 6H); ${ }^{13} \mathrm{C} \mathrm{NMR}\left(\mathrm{CDCl}_{3}, 100 \mathrm{MHz}\right)$ 140.34, 138.99, 132.94, 129.00, 128.60, 128.05, 126.43, 97.70, 75.86, 66.33, 59.52, 25.86, 18.19, 5.21; IR (film) 2927.9, 2855.5, 1697.8, 1565.7, 1462.9, 1435.9, 1253.9, 1200.0, 115.2, 1088.5, 1012.7, 837.3, 778.8, 750.1 $\mathrm{cm}^{-1}$; HRMS-ESI $(\mathrm{m} / \mathrm{z})$ : $[\mathrm{M}+\mathrm{Na}]^{+}$calcd for $\mathrm{C}_{17} \mathrm{H}_{27} \mathrm{O}_{2} \mathrm{ISiNa} 441.0728$, found 441.0751 . 


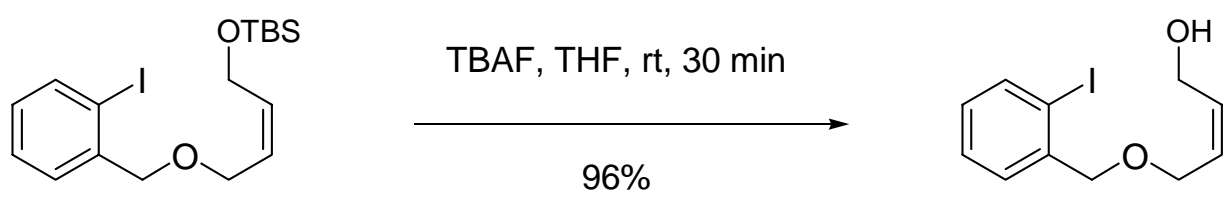

To a solution of the substrate $(0.76 \mathrm{~g}, 1.9 \mathrm{mmol})$ in THF $(20 \mathrm{~mL})$ was added a solution of tetrabutylammonium fluoride $1 \mathrm{M}$ in THF $(2 \mathrm{~mL})$. The mixture was stirred for 30 minutes at room temperature. The reaction was quenched with water. The aqueous layer was extracted with diethyl ether. The organic layer was dried over $\mathrm{Na}_{2} \mathrm{SO}_{4}$ and concentrated under reduced pressure. The residue was purified by chromatography over silica gel (Hexanes:EtOAc, 9:1) to give the desired compound $(0.57 \mathrm{~g}, 96 \%)$ as a colorless viscous oil.

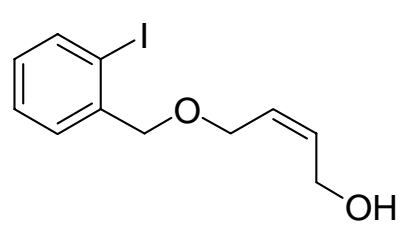

4-(2-Iodo-benzyloxy)-but-2-en-1-ol: colorless viscous oil, purified by chromatography over silica gel $(96 \%$,$) , Hexanes:EtOAc,$ 9:1, $\mathrm{Rf}=0.13) ;{ }^{1} \mathrm{H} \mathrm{NMR}\left(\mathrm{CDCl}_{3}, 400 \mathrm{MHz}\right) 7.82(\mathrm{~d}, J=7.7 \mathrm{~Hz}$, $1 \mathrm{H}), 7.41(\mathrm{~d}, J=7.7 \mathrm{~Hz}, 1 \mathrm{H}), 7.34(\mathrm{t}, J=7.7 \mathrm{~Hz}, 1 \mathrm{H}), 6.99(\mathrm{t}, J=$ $7.7 \mathrm{~Hz}, 1 \mathrm{H}), 5.87-5.73(\mathrm{~m}, 2 \mathrm{H}), 4.51(\mathrm{~s}, 2 \mathrm{H}), 4.21(\mathrm{~d}, J=6.2 \mathrm{~Hz}, 2 \mathrm{H}), 4.17(\mathrm{~d}, J=5.8 \mathrm{~Hz}, 2 \mathrm{H})$, 2.13 (s br, OH); ${ }^{13} \mathrm{C}$ NMR $\left(\mathrm{CDCl}_{3}, 100 \mathrm{MHz}\right) 140.08,139.20,132.45,129.31,128.85$, 128.24, 127.97, 97.99, 76.16, 66.16, 58.68; IR (film) 3385.5, 3022.4, 3055.3, 2861.9, 1719.8, $1694.4,1585.7,1564.4,1464.3,1436.9,1205.3,1118.9,1088.5,1012.7,749.7 \mathrm{~cm}^{-1}$; HRMS-ESI (m/z): $[\mathrm{M}+\mathrm{Na}]^{+}$calcd for $\mathrm{C}_{11} \mathrm{H}_{13} \mathrm{O}_{2} \mathrm{INa} 326.9863$, found 326.9860 .
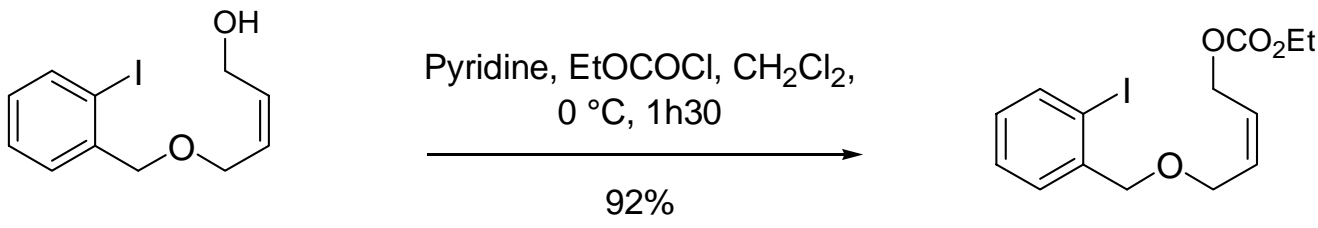

5

To a solution of substrate $(0.5 \mathrm{~g}, 1.65 \mathrm{mmol})$ in $\mathrm{CH}_{2} \mathrm{Cl}_{2}(5 \mathrm{~mL})$ were added pyridine $(0.2$ $\mathrm{mL}, 2.50 \mathrm{mmol})$ and ethylchloroformate $(0.19 \mathrm{~mL}, 1.98 \mathrm{mmol})$ at $0{ }^{\circ} \mathrm{C}$. The reaction mixture was stirred at $0{ }^{\circ} \mathrm{C}$ for $1 \mathrm{~h} 30$, warmed up at room temperature and diluted with $\mathrm{CH}_{2} \mathrm{Cl}_{2}$. The organic layer was washed with $\mathrm{HCl} 1 \mathrm{~N}$, with a saturated solution of $\mathrm{NaHCO}_{3}$ and brine. The 
organic layer was dried over $\mathrm{Na}_{2} \mathrm{SO}_{4}$ and concentrated under reduced pressure. The residue was purified by chromatography over silica gel (Hexanes:EtOAc, 97:3) to give the desired compound $(0.57 \mathrm{~g}, 92 \%)$ as a colorless viscous oil.

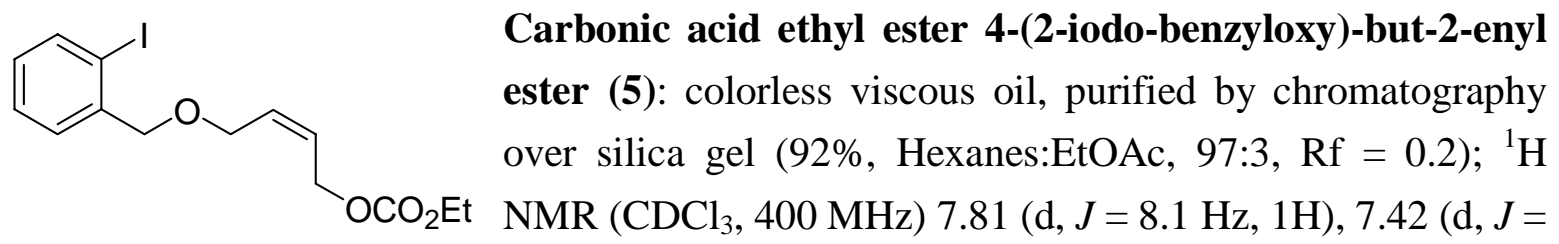
$7.7 \mathrm{~Hz}, 1 \mathrm{H}), 7.33(\mathrm{t}, J=7.7 \mathrm{~Hz}, 1 \mathrm{H}), 6.97(\mathrm{t}, J=7.7 \mathrm{~Hz}, 1 \mathrm{H}), 5.91-5.72(\mathrm{~m}, 2 \mathrm{H}), 4.72(\mathrm{~d}, J=$ $6.6 \mathrm{~Hz}, 2 \mathrm{H}), 4.49(\mathrm{~m}, 2 \mathrm{H}), 4.22(\mathrm{~d}, J=6.2 \mathrm{~Hz}, 2 \mathrm{H}), 4.19(\mathrm{q}, J=7.3 \mathrm{~Hz}, 2 \mathrm{H}), 1.30$ (t, $J=7.3$ $\mathrm{Hz}, 3 \mathrm{H}) ;{ }^{13} \mathrm{C} \mathrm{NMR}\left(\mathrm{CDCl}_{3}, 100 \mathrm{MHz}\right) 154.71,139.98,138.89,130.79,128.98,128.51$, 127.97, 126.07, 97.61, 75.91, 66.00, 63.76, 63.10, 14.07; IR (film) 2982.5, 2935.5, 2866.8, $1747.5,1465.5,1438.7,1373.2,1262.0,1090.8,1013.3,873.6,752.3 \mathrm{~cm}^{-1}$; HRMS-ESI $(\mathrm{m} / \mathrm{z}):[\mathrm{M}+\mathrm{H}]^{+}$calcd for $\mathrm{C}_{14} \mathrm{H}_{17} \mathrm{O}_{4} \mathrm{I} 377.0244$, found 377.0256 .
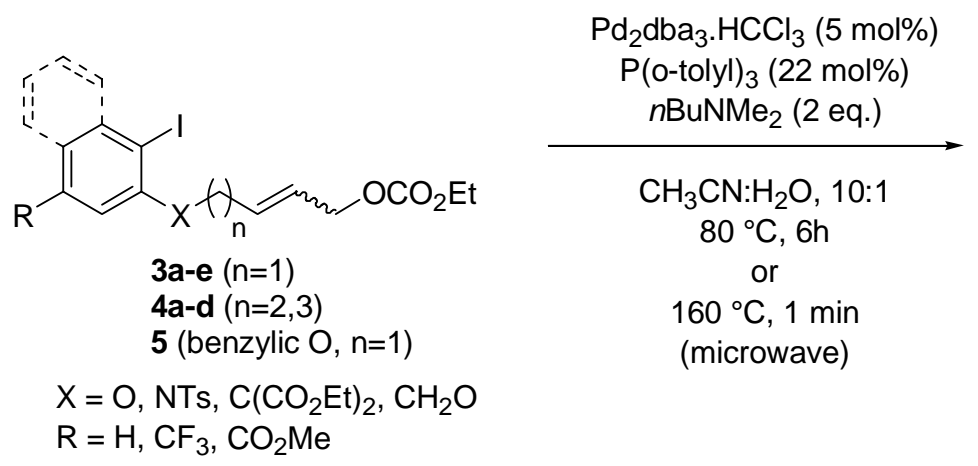

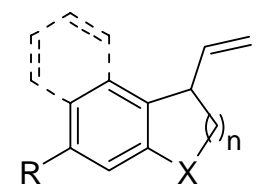

6a-j

Conventional thermal conditions: To a mixture of 3a-e or $\mathbf{4 a - d}$ or $\mathbf{5}$ (1.0 equiv), $(o \text {-tolyl })_{3} \mathrm{P}$ (22 mol\%) and $n \mathrm{BuNMe}_{2}$ (2.0 equiv) in $\mathrm{CH}_{3} \mathrm{CN}: \mathrm{H}_{2} \mathrm{O}(10: 1,0.1 \mathrm{M})$ was added $\mathrm{Pd}_{2}(\mathrm{dba})_{3} \cdot \mathrm{CHCl}_{3}(5 \mathrm{~mol} \%)$. The resulting mixture was degassed by Ar bubling and was refluxed for $6 \mathrm{~h}$ under $\mathrm{N}_{2}$ atmosphere. The mixture was cooled at room temperature and treated with $\mathrm{H}_{2} \mathrm{O}$. The aqueous layer was extracted with $\mathrm{CH}_{2} \mathrm{Cl}_{2}$. The organic layer was washed with water, brine and was dried over $\mathrm{Na}_{2} \mathrm{SO}_{4}$. After concentration under reduced pressure, the resulting residue was purified over silica gel using mixtures of hexanes or pentane/ $\mathrm{Et}_{2} \mathrm{O}$ as eluent.

Microwave-assisted reaction: the reactants were filled into an appropriate microwave 
process vial sealed with a Teflon septum. The mixture was degassed by Ar bubling and was heated at $160{ }^{\circ} \mathrm{C}$ for 1 minute (pressure $\sim 8$ bars). After microwave irradiation and subsequent gas jet cooling (down to $40{ }^{\circ} \mathrm{C}$ ), the reaction mixture was quenched with water. The workup described above for conventional thermal conditions was then realized.

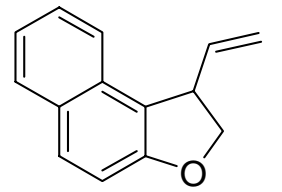

1-Vinyl-1,2-dihydro-naphtho[2,1-b]furan (6a): colorless viscous oil, purified by chromatography over silica gel (56\% (microwave irradiation), $33 \%$ (conventional thermal conditions), Hexanes: $\left.\mathrm{Et}_{2} \mathrm{O}, 99: 1, \mathrm{Rf}=0.4\right) ;{ }^{1} \mathrm{H}$ $\operatorname{NMR}\left(\mathrm{CDCl}_{3}, 400 \mathrm{MHz}\right) 7.80(\mathrm{~d}, J=8.1 \mathrm{~Hz}, 1 \mathrm{H}), 7.77(\mathrm{~d}, J=8.1 \mathrm{~Hz}, 1 \mathrm{H}), 7.70(\mathrm{~d}, J=8.8$ $\mathrm{Hz}, 1 \mathrm{H}), 7.43$ (t, $J=8.4 \mathrm{~Hz}, 1 \mathrm{H}), 7.29$ (t, $J=8.1 \mathrm{~Hz}, 1 \mathrm{H}), 7.12(\mathrm{~d}, J=8.8 \mathrm{~Hz}, 1 \mathrm{H}), 6.06-5.97$ (m, 1H), $5.28(\mathrm{~d}, J=17.2 \mathrm{~Hz}, 1 \mathrm{H}), 5.18(\mathrm{~d}, J=10.2 \mathrm{~Hz}, 1 \mathrm{H}), 4.87-4.80$ (m, 1H), 4.51-4.43 (m, $2 \mathrm{H}) ;{ }^{13} \mathrm{C} \mathrm{NMR}\left(\mathrm{CDCl}_{3}, 100 \mathrm{MHz}\right) 157.54,138.66,130.90,129.68,129.38,128.60,126.42$, 122.68, 122.32, 119.49, 116.08, 112.01, 76.51, 46.38; IR (film) 3067, 2971, 2888, 1630, 1597, 1579, 1519, 1473, 1459, 1372, 1246, 1156, 1058, 989, 950, 921, 810, $745 \mathrm{~cm}^{-1}$; HRMS-EI (m/z): $\left[\mathrm{M}^{+}\right]$calcd for $\mathrm{C}_{14} \mathrm{H}_{12} \mathrm{O} 196.088815$, found 196.089237.

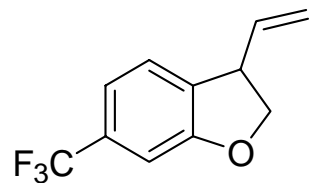

6-Trifluoromethyl-3-vinyl-2,3-dihydro-benzofuran (6b): colorless viscous oil, purified over thin layer chromatography plate of $\mathrm{SiO}_{2}(61 \%$ (microwave irradiation), 20\% (conventional thermal conditions), Pentane:Et $\left.{ }_{2} \mathrm{O}, 99: 1, \mathrm{Rf}=0.6\right) ;{ }^{1} \mathrm{H} \mathrm{NMR}\left(\mathrm{CDCl}_{3}, 400 \mathrm{MHz}\right) 7.20-7.13(\mathrm{~m}, 2 \mathrm{H}), 7.02(\mathrm{~s}, 1 \mathrm{H})$, 5.89-5.81 (ddd, $J=17.2 \mathrm{~Hz}, J=10.2 \mathrm{~Hz}, J=8.1 \mathrm{~Hz}, 1 \mathrm{H}), 5.27-5.19(\mathrm{~m}, 2 \mathrm{H}), 4.78(\mathrm{t}, J=9.1$ $\mathrm{Hz}, 1 \mathrm{H}), 4.31(\mathrm{t}, J=8.8 \mathrm{~Hz}, 1 \mathrm{H}), 4.16(\mathrm{q}, J=9.1 \mathrm{~Hz}, 1 \mathrm{H}) ;{ }^{13} \mathrm{C} \mathrm{NMR}\left(\mathrm{CDCl}_{3}, 125 \mathrm{MHz}\right)$ 160.06, 136.79, 133.41, $131.06(\mathrm{q}, J=32.7 \mathrm{~Hz}), 125.10,121.8(\mathrm{q}, J=272 \mathrm{~Hz}), 117.77(\mathrm{q}, J=$ $3.8 \mathrm{~Hz}$ ), 117.46, 106.70 (q, $J=3.8 \mathrm{~Hz}$ ), 76.84, 46.62; IR (film) 2959.4, 2922.5, 2848.7, 1728.9, 1693.3, 1625.7, 1597.6, 1503.6, 1435.5, 1320.0, 1170.2, 1126.3, 1052.5, 978.8, $827.6 \mathrm{~cm}^{-1}$; HRMS-EI (m/z): $\left[\mathrm{M}^{+}\right]$calcd for $\mathrm{C}_{11} \mathrm{H}_{9} \mathrm{OF}_{3} 214.060550$, found 214.060721 .

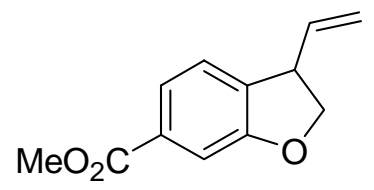

3-Vinyl-2,3-dihydro-benzofuran-6-carboxylic acid methyl ester (6c): colorless viscous oil, purified by chromatography over silica gel (66\% (microwave irradiation), 36\% (conventional thermal conditions), Pentane:Et $\left.{ }_{2} \mathrm{O}, 95: 5, \mathrm{Rf}=0.3\right) ;{ }^{1} \mathrm{H} \mathrm{NMR}\left(\mathrm{CDCl}_{3}, 400 \mathrm{MHz}\right) 7.59$ (dd, $J=7.7 \mathrm{~Hz}$, $J=1.4 \mathrm{~Hz}, 1 \mathrm{H}), 7.41(\mathrm{~d}, J=1.4 \mathrm{~Hz}, 1 \mathrm{H}), 7.15(\mathrm{~d}, J=7.7 \mathrm{~Hz}, 1 \mathrm{H}), 5.88-5.80(\mathrm{ddd}, J=17.2 \mathrm{~Hz}$, $J=10.2 \mathrm{~Hz}, J=8.1 \mathrm{~Hz}, 1 \mathrm{H}), 5.22(\mathrm{~d}, J=17.2 \mathrm{~Hz}, 1 \mathrm{H}), 5.18(\mathrm{~d}, J=10.2 \mathrm{~Hz}, 1 \mathrm{H}), 4.75(\mathrm{t}, J=$ 
$9.1 \mathrm{~Hz}, 1 \mathrm{H}), 4.28(\mathrm{t}, J=8.8 \mathrm{~Hz}, 1 \mathrm{H}), 4.14(\mathrm{q}, J=8.8 \mathrm{~Hz}, 1 \mathrm{H}), 3.88(\mathrm{~s}, 3 \mathrm{H}) ;{ }^{13} \mathrm{C} \mathrm{NMR}\left(\mathrm{CDCl}_{3}\right.$, $100 \mathrm{MHz}) 166.86,160.02$, 136.94, 134.77, 130.83, 124.57, 122.61, 117.34, 110.48, 76.60, 52.10, 46.82; IR (film) 2953, 2895, 1721, 1591, 1493, 1437, 1288, 1262, 1216, 1107, 1080, 989, 802, $762 \mathrm{~cm}^{-1}$; HRMS-EI $(\mathrm{m} / \mathrm{z})$ : $\left[\mathrm{M}^{+}\right]$calcd for $\mathrm{C}_{12} \mathrm{H}_{12} \mathrm{O}_{3} 204.078644$, found 204.078155 .

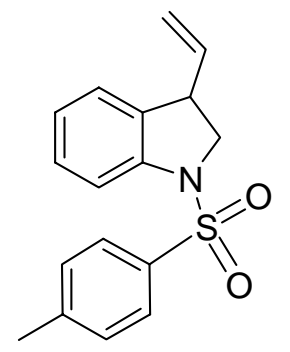

1-(Toluene-4-sulfonyl)-3-vinyl-2,3-dihydro-1H-indole (6d): white solid, purified by chromatography over silica gel and by subsequent treatment with hydroxylamine hydrochloride and sodium acetate in $\mathrm{MeOH}$ to remove traces of dibenzylidene-acetone (74\% in both conditions, Hexanes: $\mathrm{Et}_{2} \mathrm{O}$, 95:5, Rf = 0.16); M.p. 99-101 ${ }^{\circ} \mathrm{C} ;{ }^{1} \mathrm{H}$ NMR (400 MHz, $\left.\mathrm{CDCl}_{3}\right)$ 7.68-7.64 $(\mathrm{m}, 3 \mathrm{H}), 7.24-7.20(\mathrm{~m}, 3 \mathrm{H}), 7.01-7.00(\mathrm{~m}, 2 \mathrm{H}), 5.58-5.49(\mathrm{~m}, 1 \mathrm{H})$, $5.10-5.04(\mathrm{~m}, 2 \mathrm{H}), 4.14(\mathrm{dd}, J=10.6 \mathrm{~Hz}, J=9.5 \mathrm{~Hz}, 1 \mathrm{H}), 3.74(\mathrm{q}, J=8.4 \mathrm{~Hz}, 1 \mathrm{H}), 3.58(\mathrm{dd}$, $J=10.6 \mathrm{~Hz}, J=7.7 \mathrm{~Hz}, 1 \mathrm{H}), 2.37(\mathrm{~s}, 3 \mathrm{H}) ;{ }^{13} \mathrm{C} \mathrm{NMR}\left(100 \mathrm{MHz}, \mathrm{CDCl}_{3}\right)$ 144.07, 141.67, 137.34, 133.89, 133.76, 129.62, 128.28, 127.32, 125.04, 123.86, 117.10, 115.07, 55.55, 44.82, 21.51; IR (film) 3064.6, 2924.3, 1638.1, 1598.5, 1476.1, 1459.0, 1355.1, 1234.3, 1168.2, 1101.6, 1091.0, 1047.7, 926.0, 813.9, $754.6 \mathrm{~cm}^{-1} ; \mathrm{HRMS}-\mathrm{EI}(\mathrm{m} / \mathrm{z}):\left[\mathrm{M}^{+}\right]$calcd for $\mathrm{C}_{17} \mathrm{H}_{17} \mathrm{NO}_{2} \mathrm{~S} 299.098001$, found 299.098227.

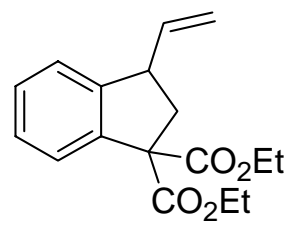

3-Vinyl-indan-1,1-dicarboxylic acid diethyl ester (6e): colorless viscous oil, purified by chromatography over silica gel (67\% (microwave irradiation), Hexanes:Et $\left.{ }_{2} \mathrm{O}, 95: 5, \mathrm{Rf}=0.27\right) ;{ }^{1} \mathrm{H} \mathrm{NMR}\left(\mathrm{CDCl}_{3}, 400 \mathrm{MHz}\right)$ 7.59-7.56 (m, 1H), 7.32-7.25 (m, 2H), 7.16-7.14 (m, 1H), 5.85-5.76 (ddd, $J=17.2 \mathrm{~Hz}, J=10.2 \mathrm{~Hz}, J=8.4 \mathrm{~Hz}, 1 \mathrm{H}), 5.23(\mathrm{~d}, J=17.2 \mathrm{~Hz}, 1 \mathrm{H}), 5.15(\mathrm{~d}, J=10.2 \mathrm{~Hz}, 1 \mathrm{H})$, 4.26 (q, $J=7.3 \mathrm{~Hz}, 2 \mathrm{H}), 4.22-4.11(\mathrm{~m}, 2 \mathrm{H}), 3.93(\mathrm{q}, J=8.4 \mathrm{~Hz}, 1 \mathrm{H}), 3.04(\mathrm{dd}, J=13.5 \mathrm{~Hz}, J$ $=7.7 \mathrm{~Hz}, 1 \mathrm{H}), 2.38(\mathrm{dd}, J=13.2 \mathrm{~Hz}, J=8.8 \mathrm{~Hz}, 1 \mathrm{H}), 1.29(\mathrm{t}, J=7.3 \mathrm{~Hz}, 3 \mathrm{H}), 1.23(\mathrm{t}, J=7.3$ $\mathrm{Hz}, 3 \mathrm{H}) ;{ }^{13} \mathrm{C} \mathrm{NMR}\left(\mathrm{CDCl}_{3}, 100 \mathrm{MHz}\right) 170.59,170.28,145.93,139.82,138.97,128.98$, 127.14, 126.57, 124.46, 116.29, 64.74, 61.69, 61.65, 47.78, 41.07, 14.07, 13.96; IR (film) 2982.1, 2929.8, 2900.3, 2878.2, 1732.1, 1474.8, 1366.8, 1250.3, 1186.5, 1097.5, 859.8, $761.2 \mathrm{~cm}^{-1}$; HRMS-EI (m/z): $\left[\mathrm{M}^{+}\right]$calcd for $\mathrm{C}_{17} \mathrm{H}_{20} \mathrm{O}_{4} 288.136159$, found 288.136099.

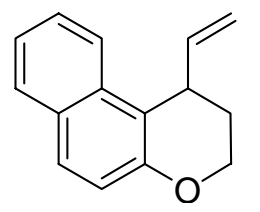

1-Vinyl -2,3-dihydro-1H-benzo[f]chromene (6f): colorless viscous oil, purified by chromatography over silica gel (62\% (microwave irradiation), 
$46 \%$ (conventional thermal conditions), Hexanes:Et $2 \mathrm{O}, 99: 1, \mathrm{Rf}=0.4) ;{ }^{1} \mathrm{H} \mathrm{NMR}\left(\mathrm{CDCl}_{3}\right.$, $400 \mathrm{MHz}) 7.80(\mathrm{~d}, J=8.8 \mathrm{~Hz}, 1 \mathrm{H}), 7.74(\mathrm{~d}, J=8.1 \mathrm{~Hz}, 1 \mathrm{H}), 7.64(\mathrm{~d}, J=8.8 \mathrm{~Hz}, 1 \mathrm{H}), 7.43$ $(\mathrm{ddd}, J=8.4 \mathrm{~Hz}, J=7.0 \mathrm{~Hz}, J=1.5 \mathrm{~Hz}, 1 \mathrm{H}), 7.30(\mathrm{ddd}, J=8.1 \mathrm{~Hz}, J=7.0 \mathrm{~Hz}, J=1.5 \mathrm{~Hz}$, $1 \mathrm{H}), 7.05(\mathrm{~d}, J=9.1 \mathrm{~Hz}, 1 \mathrm{H}), 6.11(\mathrm{ddd}, J=17.2 \mathrm{~Hz}, J=10.2 \mathrm{~Hz}, J=5.5 \mathrm{~Hz}, 1 \mathrm{H}), 5.20(\mathrm{dt}$, $J=10.2 \mathrm{~Hz}, J=1.5 \mathrm{~Hz}, 1 \mathrm{H}), 4.81(\mathrm{dt}, J=17.2 \mathrm{~Hz}, J=1.5 \mathrm{~Hz}, 1 \mathrm{H}), 4.33-4.28(\mathrm{~m}, 1 \mathrm{H})$, 4.24-4.18 (m, 1H), 4.07-4.04 (m, 1H), 2.31-2.22 (m, 1H), 2.01-1.96 (m, 1H); ${ }^{13} \mathrm{C} \mathrm{NMR}$ $\left(\mathrm{CDCl}_{3}, 100 \mathrm{MHz}\right) 152.33,141.01,133.03,128.99,128.51,128.33,126.11,122.91,122.88$, 118.96, 117.65, 114.06, 61.74, 34.12, 27.28; IR (film) 3062.0, 2972.4, 2880.9, 1622.0, 1599.3, 1514.1, 1474.2, 1433.3, 1403.2, 1285.9, 1264.9, 1235.7, 1211.9, 1083.6, 1002.6, 921.6, 812.2 $\mathrm{cm}^{-1}$; HRMS-EI (m/z): $\left[\mathrm{M}^{+}\right]$calcd for $\mathrm{C}_{15} \mathrm{H}_{14} \mathrm{O} 210.104465$, found 210.105084 .

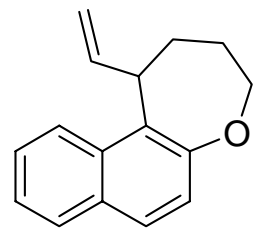

1-Vinyl-1,2,3,4-tetrahydro-naphtho[2,1-b]oxepine (6g): colorless viscous oil, purified by chromatography over silica gel (72\% (microwave irradiation), $49 \%$ (conventional thermal conditions), Hexanes: $\mathrm{Et}_{2} \mathrm{O}, 99: 1, \mathrm{Rf}$ $=0.4) ;{ }^{1} \mathrm{H} \mathrm{NMR}\left(\mathrm{CDCl}_{3}, 400 \mathrm{MHz}\right) 7.98(\mathrm{~d}, J=8.4 \mathrm{~Hz}, 1 \mathrm{H}), 7.78(\mathrm{~d}, J=8.1$ $\mathrm{Hz}, 1 \mathrm{H}), 7.64(\mathrm{~d}, J=8.8 \mathrm{~Hz}, 1 \mathrm{H}), 7.46(\mathrm{ddd}, J=8.4 \mathrm{~Hz}, \mathrm{~J}=7.0 \mathrm{~Hz}, J=1.5 \mathrm{~Hz}, 1 \mathrm{H}), 7.35$ (ddd, $J=8.1 \mathrm{~Hz}, J=7.0 \mathrm{~Hz}, J=1.1 \mathrm{~Hz}, 1 \mathrm{H}), 7.17(\mathrm{~d}, J=8.8 \mathrm{~Hz}, 1 \mathrm{H}), 6.29(\mathrm{ddd}, J=17.2 \mathrm{~Hz}, J=$ $10.2 \mathrm{~Hz}, J=5.9 \mathrm{~Hz}, 1 \mathrm{H}), 5.13(\mathrm{td}, J=10.2 \mathrm{~Hz}, J=1.5 \mathrm{~Hz}, 1 \mathrm{H}), 4.93(\mathrm{td}, J=17.2 \mathrm{~Hz}, J=1.8$ $\mathrm{Hz}, 1 \mathrm{H}), 4.51-4.44(\mathrm{~m}, 1 \mathrm{H}), 4.42-4.36(\mathrm{~m}, 1 \mathrm{H}), 3.99-3.94(\mathrm{~m}, 1 \mathrm{H}), 2.22-1.94(\mathrm{~m}, 4 \mathrm{H}) ;{ }^{13} \mathrm{C}$ $\mathrm{NMR}\left(\mathrm{CDCl}_{3}, 100 \mathrm{MHz}\right) 158.01,140.23,133.36,130.66,128.49,128.27,126.20,126.09$, 123.77, 123.49, 122.51, 115.94, 72.53, 41.37, 29.63, 26.66; IR (film) 3064.8, 2935.8, 2860.0, 1636.4, 1620.3, 1596.8, 1508.8, 1470.0, 1429.5, 1382.4, 1241.5, 1220.1, 1159.4, 1047.9, 952.0, 918.5, 821.1, 748.1 $\mathrm{cm}^{-1}$; HRMS-EI (m/z): $\left[\mathrm{M}^{+}\right]$calcd for $\mathrm{C}_{16} \mathrm{H}_{16} \mathrm{O} 224.120115$, found 224.120409.

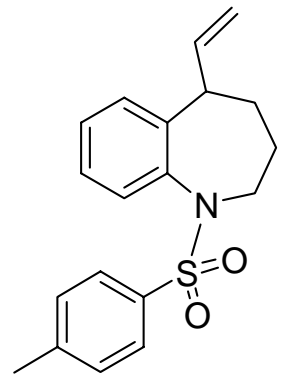

\section{1-(Toluene-4-sulfonyl)-5-vinyl-2,3,4,5-tetrahydro-1H-benzo[b]azepine}

(6h): white solid, purified by chromatography over silica gel purified by chromatography over silica gel and by subsequent treatment with hydroxylamine hydrochloride and sodium acetate in $\mathrm{MeOH}$ to remove traces of dibenzylidene-acetone $(65 \%$ (microwave irradiation), Hexanes:Et $2 \mathrm{O}, 95: 5, \mathrm{Rf}=0.15)$; M.p. $127-129{ }^{\circ} \mathrm{C} ;{ }^{1} \mathrm{H}$ NMR $\left(\mathrm{DMSO}_{6} \mathrm{~d}_{6}\right.$, $\left.500 \mathrm{MHz}, 100{ }^{\circ} \mathrm{C}\right) 7.64(\mathrm{~d}, J=8.3 \mathrm{~Hz}, 2 \mathrm{H}), 7.39(\mathrm{~d}, J=7.8 \mathrm{~Hz}, 2 \mathrm{H}), 7.24-7.20(\mathrm{~m}, 1 \mathrm{H})$, 7.19-7.18 (m, 2H), $7.14(\mathrm{~d}, J=8.3 \mathrm{~Hz}, 1 \mathrm{H}), 6.05(\mathrm{ddd}, J=17.2 \mathrm{~Hz}, J=10.2 \mathrm{~Hz}, J=8.1 \mathrm{~Hz}$, 
$1 \mathrm{H}), 5.07(\mathrm{~d}, J=10.2 \mathrm{~Hz}, 1 \mathrm{H}), 4.87(\mathrm{~d}, J=17.2 \mathrm{~Hz}, 1 \mathrm{H}), 3.77-3.72(\mathrm{~m}, 1 \mathrm{H}), 3.43-3.38(\mathrm{~m}$, $1 \mathrm{H}), 3.21-3.18(\mathrm{~m}, 1 \mathrm{H}), 2.40(\mathrm{~s}, 3 \mathrm{H}), 1.84-1.69(\mathrm{~m}, 2 \mathrm{H}), 1.66-1.59(\mathrm{~m}, 1 \mathrm{H}), 1.50-1.41(\mathrm{~m}$, 1H); ${ }^{13} \mathrm{C}$ NMR (DMSO-d $6,125 \mathrm{MHz}, 100{ }^{\circ} \mathrm{C}$ ) 142.57, 141.27, 139.31, 138.11, 137.73, 129.09, 127.82, 127.02, 126.17, 126.15, 114.21, 48.92, 44.16, 29.93, 25.41, 20.28; IR (film) 3070.9, 2927.0, 2853.9, 1598.1, 1486.0, 1449.9, 1345.4, 1158.8, 1092.4, 1037.8, 917.4, 839.9, $713.7 \mathrm{~cm}^{-1}$; HRMS-ESI $(\mathrm{m} / \mathrm{z}):[\mathrm{M}+\mathrm{H}]^{+}$calcd for $\mathrm{C}_{19} \mathrm{H}_{22} \mathrm{NO}_{2} \mathrm{~S} 328.1365$, found 328.1370 .

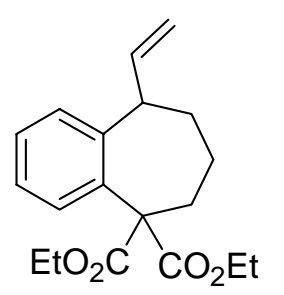

9-Vinyl-6,7,8,9-tetrahydro-benzocycloheptene-5,5-dicarboxylic acid diethyl ester (6i): colorless viscous oil, purified by chromatography over silica gel (73\% (microwave irradiation), Hexanes: $\left.\mathrm{Et}_{2} \mathrm{O}, 95: 5, \mathrm{Rf}=0.2\right) ;{ }^{1} \mathrm{H}$ NMR $\left(\mathrm{CDCl}_{3}, 400 \mathrm{MHz}\right) 7.22-7.21(\mathrm{~m}, 2 \mathrm{H}), 7.18-7.13(\mathrm{~m}, 1 \mathrm{H}), 6.96(\mathrm{~d}, J=$ $=10.2 \mathrm{~Hz}, J=1.5 \mathrm{~Hz}, 1 \mathrm{H}), 5.06(\mathrm{td}, J=17.2 \mathrm{~Hz}, J=1.5 \mathrm{~Hz}, 1 \mathrm{H}), 4.37-4.22(\mathrm{~m}, 4 \mathrm{H})$, 3.47-3.43 (m, 1H), 2.53-2.47 (m, 1H), 2.28-2.21 (m, 1H), 1.93-1.80 (m, 3H), 1.56-1.48 (m, $1 \mathrm{H}), 1.30(\mathrm{t}, J=7.3 \mathrm{~Hz}, 3 \mathrm{H}), 1.25(\mathrm{t}, J=7.3 \mathrm{~Hz}, 3 \mathrm{H}) ;{ }^{13} \mathrm{C} \mathrm{NMR}\left(\mathrm{CDCl}_{3}, 100 \mathrm{MHz}\right) 172.71$, 171.24, 143.45, 141.02, 137.23, 128.90, 128.14, 127.41, 125.99, 114.58, 64.86, 61.75, 61.59, 46.10, 33.38, 31.16, 24 .31, 13.98, 13.94; IR (film) 3071.6, 2979.9, 2935.6, 2869.1, 1731.6, 1640.0, 1488.0, 1445.8, 1389.2, 1365.8, 1232.5, 1174.8, 1096.4, 1030.1, 916.6, $750.0 \mathrm{~cm}^{-1}$; HRMS-EI (m/z): [ $\left.\mathrm{M}^{+}\right]$calcd for $\mathrm{C}_{19} \mathrm{H}_{24} \mathrm{O}_{4} 316.167460$, found 316.167381 .

4-Vinyl-isochroman $(\mathbf{6 j})$ : colorless viscous oil, purified by chromatography
over silica gel (45\% (conventional thermal conditions), Pentane: $\mathrm{Et}_{2} \mathrm{O}, 95: 5, \mathrm{Rf}$ 5.91-5.82 (m, 1H), 5.22-5.18 (m, 2H), 4.84-4.75 (m, 2H), $4.01(\mathrm{dd}, J=11.4 \mathrm{~Hz}, J=5.0 \mathrm{~Hz}$, $1 \mathrm{H}), 3.78(\mathrm{dd}, J=11.4 \mathrm{~Hz}, J=6.5 \mathrm{~Hz}, 1 \mathrm{H}), 3.57-3.52(\mathrm{~m}, 1 \mathrm{H}) ;{ }^{13} \mathrm{C} \mathrm{NMR}\left(\mathrm{CDCl}_{3}, 100 \mathrm{MHz}\right)$ 138.66, 135.15, 134.34, 128.93, 126.58, 126.48, 124.27, 117.23, 69.87, 68.33, 42.90; IR (film) 3076.5, 2961.5, 2845.6, 1638.8, 1488.6, 1451.4, 1417,8, 1380.4, 1316.2, 1205.1, $1103.5,995.5,922.3,848.8,747.4 \mathrm{~cm}^{-1}$. 